\title{
Synchrotron-based coherent scatter $x$-ray projection imaging using an array of monoenergetic pencil beams
}

by

Karl Landheer

A thesis submitted to

the Faculty of Graduate and Postdoctoral Affairs

in partial fulfillment of

the requirements for the degree of

Master of Science

Ottawa-Carleton Institute for Physics

Department of Physics

Carleton University

Ottawa, Ontario

July 21,2012

(C) 2012, Karl Landheer 
Library and Archives

Canada

Published Heritage

Branch

395 Wellington Street

Ottawa ON K1A ON4

Canada
Bibliothèque et

Archives Canada

Direction du

Patrimoine de l'édition

395 , rue Wellington

Ottawa ON K1A ON4

Canada
Your file Votre référence

ISBN: $978-0-494-93585-9$

Our file Notre référence

ISBN: 978-0-494-93585-9
NOTICE:

The author has granted a nonexclusive license allowing Library and Archives Canada to reproduce, publish, archive, preserve, conserve, communicate to the public by telecommunication or on the Internet, loan, distrbute and sell theses worldwide, for commercial or noncommercial purposes, in microform, paper, electronic and/or any other formats.

The author retains copyright ownership and moral rights in this thesis. Neither the thesis nor substantial extracts from it may be printed or otherwise reproduced without the author's permission.
AVIS:

L'auteur a accordé une licence non exclusive permettant à la Bibliothèque et Archives Canada de reproduire, publier, archiver, sauvegarder, conserver, transmettre au public par télécommunication ou par l'Internet, prêter, distribuer et vendre des thèses partout dans le monde, à des fins commerciales ou autres, sur support microforme, papier, électronique et/ou autres formats.

L'auteur conserve la propriété du droit d'auteur et des droits moraux qui protege cette thèse. $\mathrm{Ni}$ la thèse ni des extraits substantiels de celle-ci ne doivent être imprimés ou autrement reproduits sans son autorisation.
In compliance with the Canadian Privacy Act some supporting forms may have been removed from this thesis.

While these forms may be included in the document page count, their removal does not represent any loss of content from the thesis.
Conformément à la loi canadienne sur la protection de la vie privée, quelques formulaires secondaires ont été enlevés de cette thèse.

Bien que ces formulaires aient inclus dans la pagination, il n'y aura aucun contenu manquant. 


\begin{abstract}
Traditional projection $x$-ray imaging utilizes only the information from the primary photons. Low-angle coherent scatter images can be acquired simultaneous to the primary images and provide additional information. A system at the Canadian Light Source synchrotron was configured which utilizes multiple $33.17 \mathrm{keV}$ pencil beams (up to five) to create both primary and coherent scatter projection images, simultaneously. The sample was scanned through the beams using an automated step-and-shoot setup. Pixels were acquired in a hexagonal lattice to maximize packing efficiency. The typical pitch was between 1.0 and $1.6 \mathrm{~mm}$. An MLEM-based iterative method was used to disentangle the overlapping information from the flat panel digital $\mathrm{x}$-ray detector. The pixel value of the coherent scatter image was generated by integrating the radial profile (scatter intensity versus scattering angle) over an angular range. Different angular ranges maximize the contrast between different materials. For a five-beam porcine phantom image the muscle-fat contrast was $0.10 \pm 0.01$ and $1.16 \pm 0.03$ for the primary and scatter images, respectively. The air kerma was measured using $\mathrm{Al}_{2} \mathrm{O}_{3}: \mathrm{C}$ optically stimulated luminescent dosimeters. The total area-averaged air kerma for the scan was measured to be $(7.2 \pm 0.4) \mathrm{cGy}$ although, due to difficulties in small-beam dosimetry, this number could be inaccurate.
\end{abstract}




\section{Acknowledgements}

I would like to thank Dr. Paul C. Johns for his continued assistance and guidance throughout all of this work.

Special thanks to Dr. George Belev, Dr. Dean Chapman and Dr. Brian Bewer for their assistance in the experimental setup at the Canadian Light Source synchrotron.

At Carleton we thank the Science Technology Centre for their construction of the collimator, Mike Antunes for his work with the assembly of the photodiodes, and David Flint and Dr. Gabriel Sawakuchi for their help with the OSLDs.

This work was supported by the Natural Sciences and Engineering Research Council of Canada (NSERC), travel assistance from Carleton University and CLSI, and by an Ontario Graduate Scholarship. Experimental research was performed at the Canadian Light Source, which is supported by NSERC, the National Research Council of

Canada, the Canadian Institutes of Health Research, the Province of Saskatchewan, Western Economic Diversification Canada, and the University of Saskatchewan. 


\section{Table of Contents}

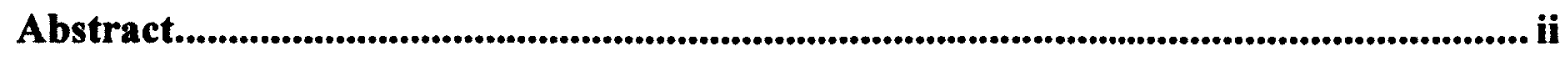

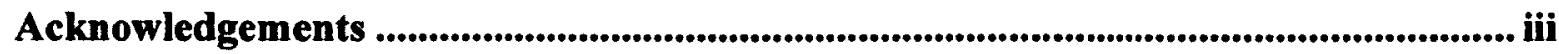

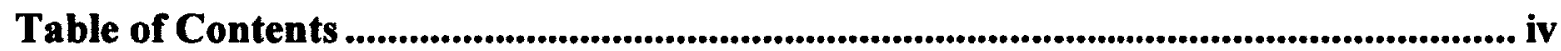

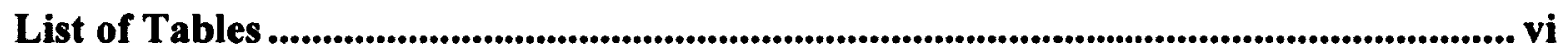

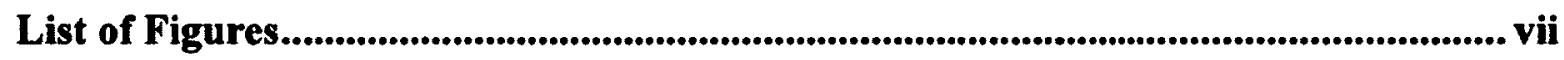

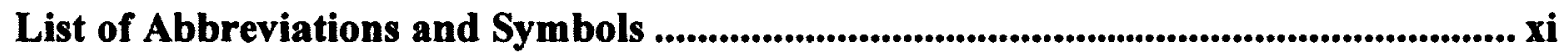

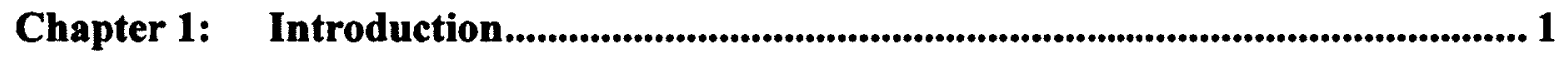

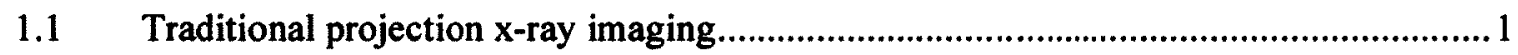

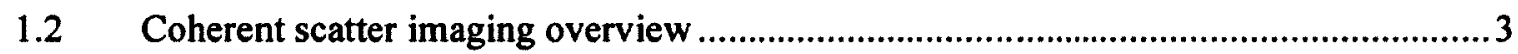

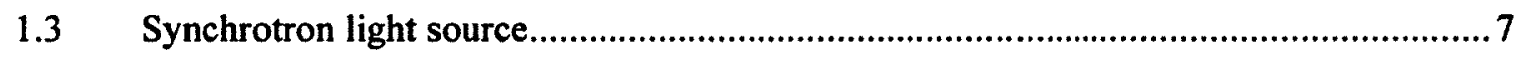

1.4 Coherent scatter imaging research at Carleton University ....................................

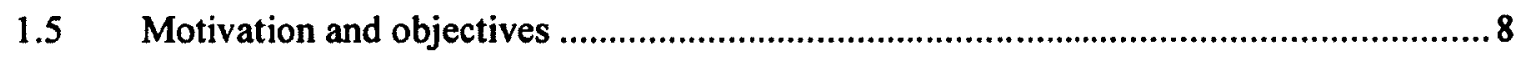

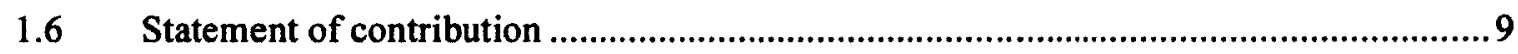

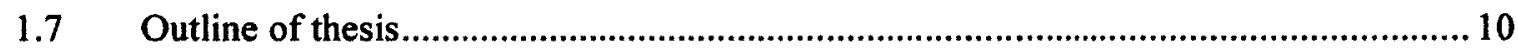

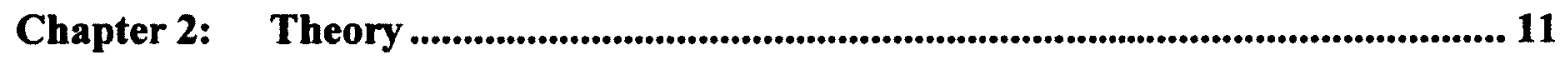

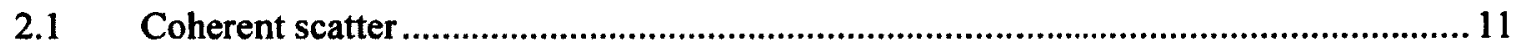

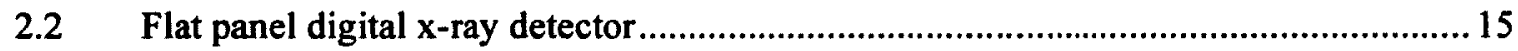

Chapter 3: Method .............................................................................................. 16

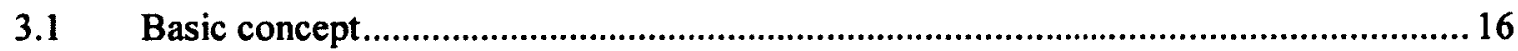

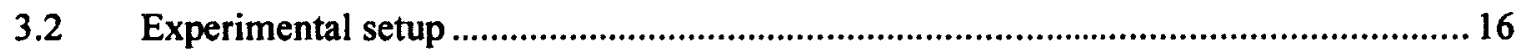

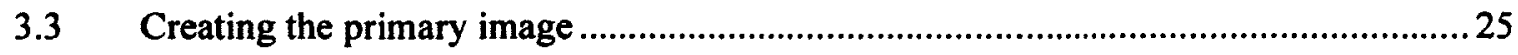

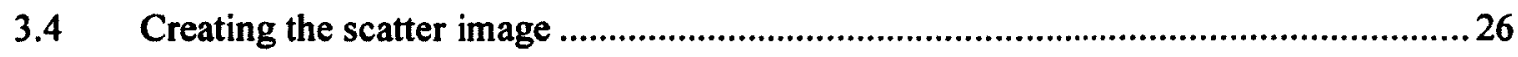

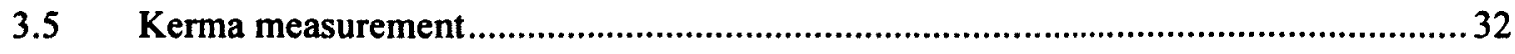

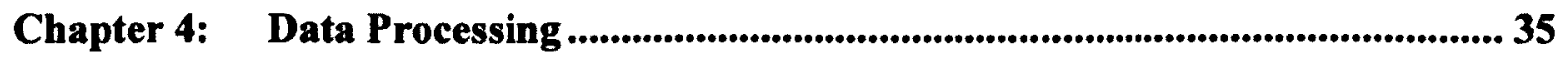




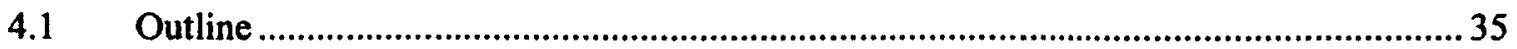

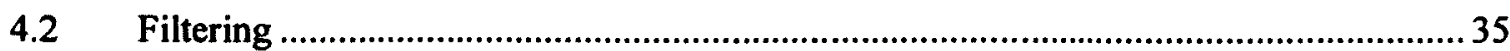

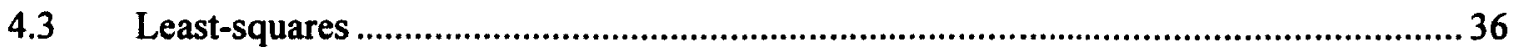

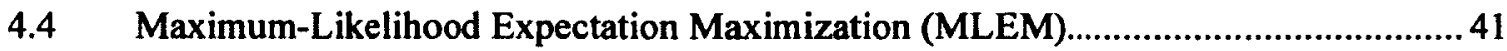

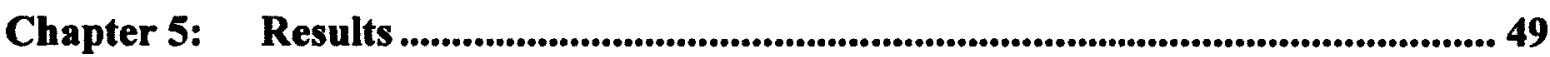

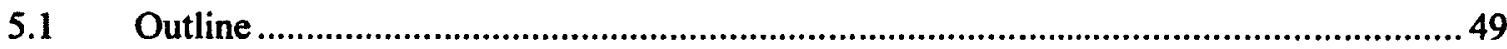

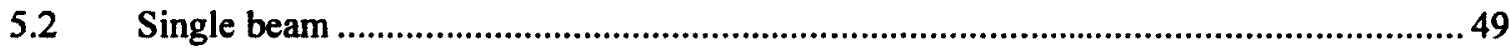

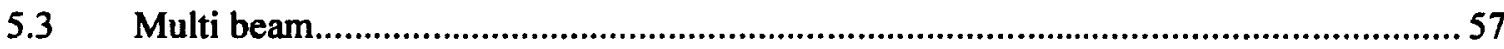

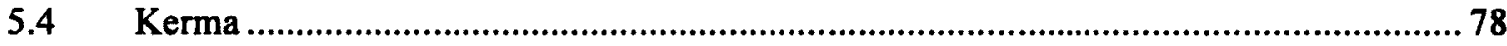

Chapter 6: Conclusions and Future Work...................................................... 81

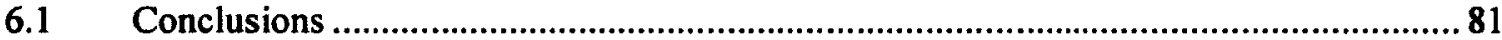

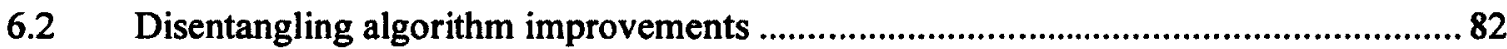

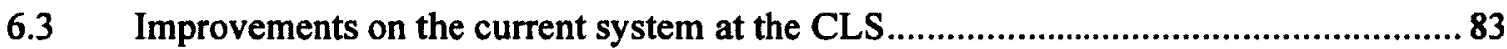

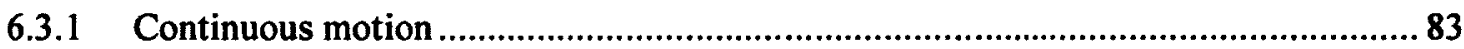

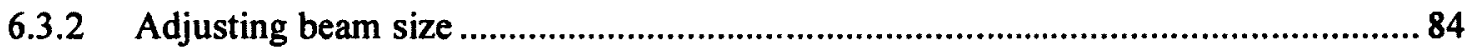

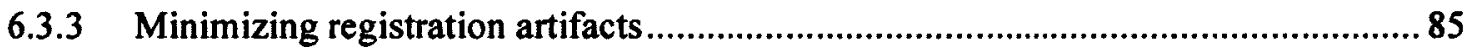

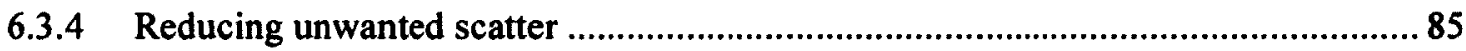

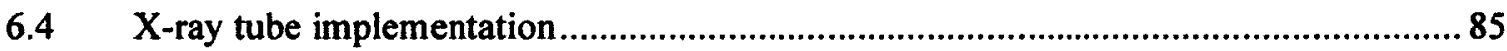

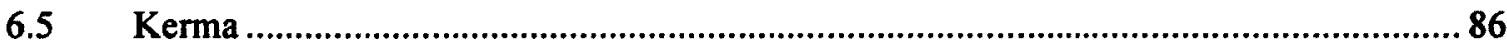

References ........................................................................................................... 88

Appendix...................................................................................................................................... 95

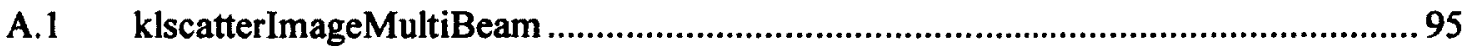

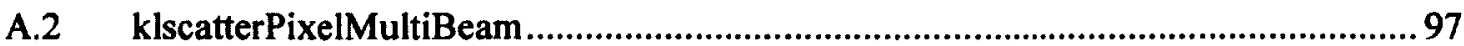

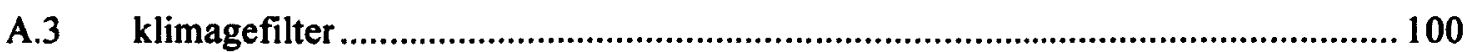

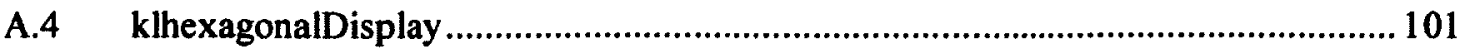




\section{List of Tables}

Table 3.1: Types of photodiodes and cables used at the CLS. .................................. 21

Table 3.2: Normalization values due to differences in the beams ................................. 29

Table 3.3: Angular ranges to maximize contrast between two biological tissues ........... 32

Table 5.1: Outline of several characteristics of images presented in this chapter.......... 50

Table 5.2: Contrast for single beam measurements for both primary and scatter (corrected for attenuation) images of porcine phantom for June 2011 experiment. ...................... 56

Table 5.3: Contrast between pairs of materials for two beam measurements for both primary and scatter (corrected for attenuation) images of tissue/plastic phantom for

December 2011 experiment.

Table 5.4: Contrast between pairs of materials for both primary and scatter (corrected for attenuation) images for single and double beam measurements of porcine phantom for

December 2011 experiment

Table 5.5: Contrast for five-beam measurements for both primary and scatter (corrected

for attenuation) images of porcine phantom for May 2012 experiment.

Table 5.6: Number of read-out photons from the OSLDs exposed at the CLS for $3.3 \mathrm{~s} . .79$ 


\section{List of Figures}

Figure 1.1: Filtered scatter pattern of $10 \mathrm{~mm}$ Lucite cuvette of ice................................... 4

Figure 1.2: Filtered scatter pattern of $10 \mathrm{~mm}$ Lucite cuvette of water ................................ 4

Figure 1.3: Filtered scatter pattern of Strontium Ranelate sachet...................................... 5

Figure 1.4: Filtered scatter pattern of a vitamin $C$ tablet dissolved in water in a $10 \mathrm{~mm}$

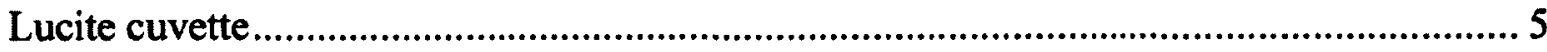

Figure 2.1: $\log -\log$ plot of photon interaction cross sections of water versus energy $\ldots . . .13$

Figure 2.2: Illustration of the process of coherent scatter............................ 14

Figure 2.3: Plot of differential cross section per $\mathrm{e}^{-}$versus scattering angle for water...... 14

Figure 3.1: Flow diagram of method to create single beam scatter images............. 17

Figure 3.2: Schematic of custom collimator base …...................................................... 18

Figure 3.3: Concept diagram of collimator with one full row of tungsten stoppers and one

empty one

Figure 3.4: Photograph of customizable collimator to convert fan beam into multiple

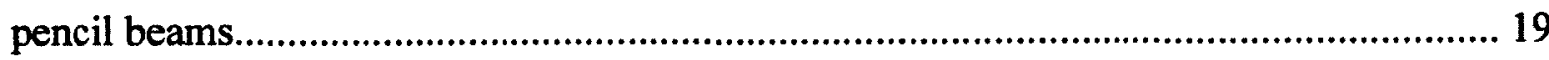

Figure 3.5: Photograph of aluminum spacer, tungsten stopper and collimator base. ....... 20

Figure 3.6: Photograph of Gafchromic film of five beams placed over grid paper.......... 20

Figure 3.7: Five photodiodes mounted on tungsten primary beam stopper....................... 22

Figure 3.8: Concept diagram for multi-beam experimental setup .................................. 23

Figure 3.9: Photograph of the experimental setup for multiple beam scatter pattern

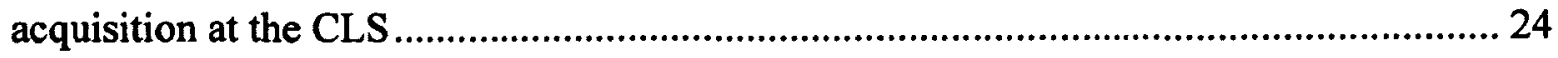

Figure 3.10: Acquisition of data scheme illustrated for two beams ................................. 25

Figure 3.11: Primary image of a standard line pair resolution pattern .............................. 26 
Figure 3.12: Photograph of uniform plastic bar used as test object

Figure 3.13: Scatter pattern of a uniform plastic bar that has been median-filtered, highpass filtered and had background subtracted 27

Figure 3.14: Three radial profiles extracted using MLEM .............................................. 28

Figure 3.15: Three radial profiles of the uniform plastic bar after normalization............ 30

Figure 3.16: Radial profiles of polyethylene and polystyrene .......................................... 31

Figure 3.17: Plot of optical read-out photons versus measured air kerma......................... 33

Figure 4.1: Median-filtered scatter pattern of a plastic bar............................................... 37

Figure 4.2: Median filtered data of a single-beam exposure of a uniform plastic bar...... 37

Figure 4.3: Radial profile of the single beam scatter pattern............................................. 38

Figure 4.4: Three radial profiles extracted using least-squares ...................................... 40

Figure 4.5: Three radial profiles extracted using non-negative least-squares .................. 41

Figure 4.6: Flow chart representation of MLEM algorithm used to obtain multiple radial

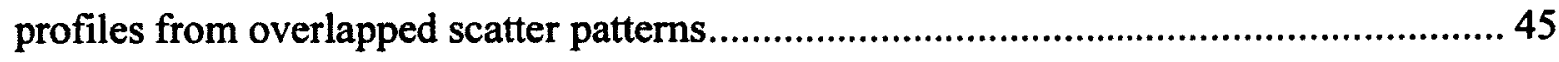

Figure 4.7: Three radial profiles extracted using MLEM ................................................. 46

Figure 4.8: Forward projection of the three MLEM-obtained radial profiles................... 46

Figure 4.9: Median-filtered five-beam exposure of uniform plastic bar .......................... 47

Figure 4.10: Five normalized radial profiles extracted from uniform plastic bar using

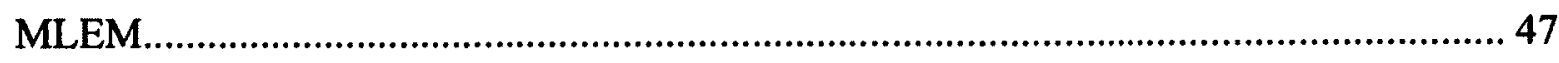

Figure 4.11: Semi-log plot of rms change from one iteration to the next versus iterations

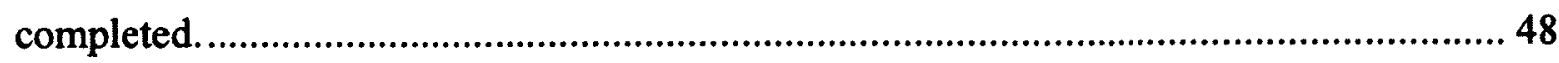

Figure 5.1: Photograph of porcine phantom (June 2011).............................................. 51

Figure 5.2: Median filtered scatter pattern of pork fat .................................................. 51 
Figure 5.3: Median filtered scatter pattern of pork muscle............................................. 52

Figure 5.4: Median filtered scatter pattern of pork cortical bone ..................................... 52

Figure 5.5: Radial profiles of fat, muscle and bone...................................................... 53

Figure 5.6: Primary image of porcine phantom ............................................................... 54

Figure 5.7: Scatter image of porcine phantom, generated by integrating the angular range $3.50^{\circ} \leq \theta \leq 5.57^{\circ}$ which maximizes the fat to muscle contrast, corrected for attenuation. 55

Figure 5.8: Scatter image of porcine phantom, generated by integrating over the entire angular range which maximizes muscle to bone contrast, corrected for attenuation ....... 56

Figure 5.9: Photograph of the tissue/plastic phantom (December 2011)......................... 58

Figure 5.10: Primary image of tissue/plastic phantom .....................................................59

Figure 5.11: Coherent scatter image of tissue/plastic phantom generated by integrating

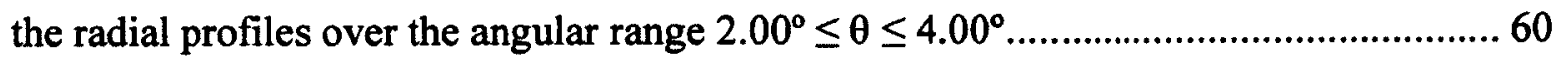
Figure 5.12: Coherent scatter image (corrected for attenuation) of tissue/plastic phantom

Figure 5.13: Coherent scatter image (corrected for attenuation) of tissue/plastic phantom windowed and levelled to reduce contrast and display a larger range of values 62

Figure 5.14: Photograph of porcine phantom (December 2011) ...................................... 64

Figure 5.15: Primary image of porcine phantom using a single beam ............................6. 65

Figure 5.16: Coherent scatter image (corrected for attenuation) of porcine phantom ..... 66

Figure 5.17: Two-beam primary image of porcine phantom ............................................6. 67

Figure 5.18: Two-beam scatter image of porcine phantom maximizing contrast between

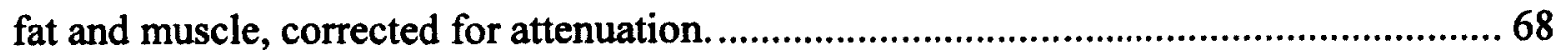

Figure 5.19: Photograph of porcine phantom (May 2012) ……...................................... 71 
Figure 5.20: One-beam scatter image maximizing fat to muscle contrast of porcine

phantom

Figure 5.21: Three-beam scatter image maximizing fat to muscle contrast of porcine

phantom.

Figure 5.22: Five-beam primary image of porcine phantom

Figure 5.23: Five-beam scatter image maximizing bone to muscle contrast of porcine

phantom.

Figure 5.24: Five-beam scatter image maximizing fat to muscle contrast of porcine

phantom.

Figure 5.25: Five-beam scatter image maximizing fat to muscle contrast corrected for

attenuation of porcine phantom

Figure 6.1: Scatter image of a Lucite step acquired with continous motion 


\section{List of Abbreviations and Symbols}

\begin{tabular}{|c|c|}
\hline $\boldsymbol{A}$ & system geometry matrix (scattering operator) \\
\hline$a$ & constant to normalize Gaussian kernel \\
\hline $\mathrm{ADC}$ & Analog-to-Digital Converter \\
\hline $\bar{b}$ & column vector of filtered data \\
\hline $\bar{C}$ & Contrast \\
\hline $\boldsymbol{c}$ & speed of light in vacuum \\
\hline $\bar{D}$ & median filtered data from the detector \\
\hline$E$ & Energy of the photon \\
\hline$F$ & coherent form factor \\
\hline$h$ & Planck's constant \\
\hline $\bar{k}$ & iteration number in MLEM algorithm \\
\hline$K_{\text {air }}$ & air kerma \\
\hline kerma & kinetic energy released per unit mass \\
\hline$l$ & width of rect function \\
\hline$L(r)$ & Likelihood of radial profile, $r$ \\
\hline$n$ & number of beams \\
\hline$N$ & Number of photons \\
\hline$m$ & length of radial profile vector \\
\hline $\bar{O}$ & number of optical read-out counts from the photomultiplier tube \\
\hline $\bar{p}$ & pixel value \\
\hline$r$ & radial profile \\
\hline $\mathrm{rms}$ & $\begin{array}{l}\text { root mean square change of radial profile from one iteration to the } \\
\text { previous }\end{array}$ \\
\hline $\boldsymbol{S}$ & median, high-pass filtered and mean of top 20 rows subtracted data \\
\hline SNR & Signal-to-Noise Ratio \\
\hline$T$ & median and high-pass filtered data \\
\hline$x$ & momentum transfer argument \\
\hline $\mathbf{x}$ & horizontal spatial coordinate \\
\hline $\mathbf{y}$ & vertical spatial coordinate \\
\hline$Z$ & atomic number \\
\hline$z$ & thickness of sample \\
\hline$\theta$ & scattering angle \\
\hline$\lambda$ & wavelength of the photon \\
\hline$\mu$ & linear attenuation coefficient \\
\hline$e \mu$ & total cross section per electron \\
\hline$\Pi$ & rect function \\
\hline$\rho$ & mass density of material \\
\hline$\sigma$ & standard deviation of Gaussian kernel \\
\hline$e_{e} \sigma_{c o h}$ & coherent cross section per electron \\
\hline$e_{i n c}$ & incoherent cross section per electron \\
\hline $\overrightarrow{e \tau}$ & photoelectric cross section per electron \\
\hline $\mathbf{\Omega}$ & solid angle \\
\hline
\end{tabular}




\section{Chapter 1: Introduction}

\subsection{Traditional projection $x-$ ray imaging}

Traditional $\mathrm{x}$-ray projection imaging is a diagnostic imaging modality which utilizes photons of energies ten to hundreds of $\mathrm{keV}$ to probe the internal structure of a patient. Applications of $\mathrm{x}$-ray imaging include medical diagnosis, non-destructive testing and security. There are three main interactions a photon can undergo in the diagnostic energy range: coherent (Rayleigh) scattering, incoherent (Compton) scattering and the photoelectric effect. ${ }^{1,2} \mathrm{X}$-ray imaging takes advantage of the interaction of photons with matter inside the target, described by the Beer-Lambert law':

$$
N=N_{0} e^{-\mu(\rho, Z) z},
$$

where $N$ is the number of photons exiting the homogenous material, $N_{0}$ is the initial number of photons impinging on the material, $\mu$ is the linear attenuation coefficient, which is dependent on the mass density, $\rho$, and atomic number $Z$, and $z$ is the thickness of the material. If the material is not homogenous then Equation 1.1 can be alternatively represented as follows:

$$
N=N_{0} e^{-\int \mu(z, \rho, z) d z} .
$$

The total cross section per electron, $e \mu$, in this energy range can be expressed as the sum of its three components: 


$$
e_{e} \mu=e_{e} \tau+{ }_{e} \sigma_{c o h}+{ }_{e} \sigma_{i n c},
$$

where $e \tau$ is the photoelectric cross section per electron, and $e^{e} \sigma_{c o h}$ and ${ }_{e} \sigma_{i n c}$ represent the coherent and incoherent contributions per electron, respectively.

It is clear from Equations 1.1 and 1.2 that regions with higher attenuation (such as bone) will allow fewer photons to emerge from the phantom or patient. Traditional transmission $\mathrm{x}$-ray imaging makes the assumption that any photon impinging on the detector has travelled straight through the patient without interaction. There is, however, evidence showing that up to $90 \%$ of the photons reaching the detector are scattered. ${ }^{3}$ Currently scattered photons are treated as a nuisance (and anti-scatter grids have been developed to minimize their effect), but in fact they carry additional information which can be utilized. Conceptually, the information carried by scatter is available without additional patient radiation dose. A clear limitation of traditional $\mathrm{x}$-ray imaging is the linear attenuation coefficients' sole dependence on density and atomic number.

Motz and Danos ${ }^{4}$ developed quantitative measures of image quality, referred to as contrast $(C)$ and signal to noise ratio (SNR). For beams through two neighbouring regions, 1 and 2:

$$
\begin{gathered}
C=2 \frac{\left|N_{1}-N_{2}\right|}{N_{1}+N_{2}}, \\
\mathrm{SNR}=\frac{\left|N_{1}-N_{2}\right|}{\sqrt{N_{1}+N_{2}}},
\end{gathered}
$$

where $N_{1}$ and $N_{2}$ are the number of photons impinging on the detector from material 1 and material 2 . These will be used as metrics for image quality throughout this work. 


\subsection{Coherent scatter imaging overview}

This work utilizes information from coherent scatter for diagnostic medical imaging. Although the coherent cross section is substantially lower than the incoherent it is mostly forward peaked making it more important in the $0-10^{\circ}$ range..$^{2,5}$ For radiography of the abdomen, behind the patient coherent first scatter is $10 \%$ of the total scatter, and the coherent first scatter is $26 \%$ of the primary fluence, in the absence of an anti-scatter grid. With a grid these numbers are reduced to $7.5 \%$ and $22 \%$, respectively. ${ }^{6}$ This is still a highly significant fraction of the total fluence. Early work by multiple groups ${ }^{6-10}$ identified coherent scatter as a source of useful diagnostic information.

Coherent scatter is dependent on the spatial positions of the electrons, which is dependent on the atomic or molecular structure of the material. Thus coherent scatter will be superior to transmission in distinguishing between materials of similar makeup. For illustration several scatter patterns are shown. Figure 1.1 is a filtered (to remove detector artifacts) scatter pattern of a $10 \mathrm{~mm}$ Lucite cuvette of ice at $33.17 \mathrm{keV}$. For comparison Figure 1.2 is that exact same cuvette after the ice had melted. Both scatter patterns had a beam-time of $16.5 \mathrm{~s}$. There are sharp peaks in the first image due to the crystalline nature of ice which are completely absent in the smoother water scatter pattern. The chemical makeup of both materials is identical, and the density of ice is only about $8 \%$ less, however due to the spatial correlation of molecules in ice versus liquid water the coherent scatter cross sections are substantially different. Figure 1.3 is a filtered scatter pattern of a Strontium Ranelate drug in a sachet exposed for $16.5 \mathrm{~s}$. By imaging the location of this chemical deposited in bone, coherent scatter could potentially be used to monitor the strontium as a tracer of bone metabolism. Figure 1.4 is a filtered scatter pattern of a 
vitamin $\mathrm{C}$ tablet dissolved in water in a $10 \mathrm{~mm}$ Lucite cuvette exposed for $33 \mathrm{~s}$. The crystalline nature can be observed from the high localized peaks.

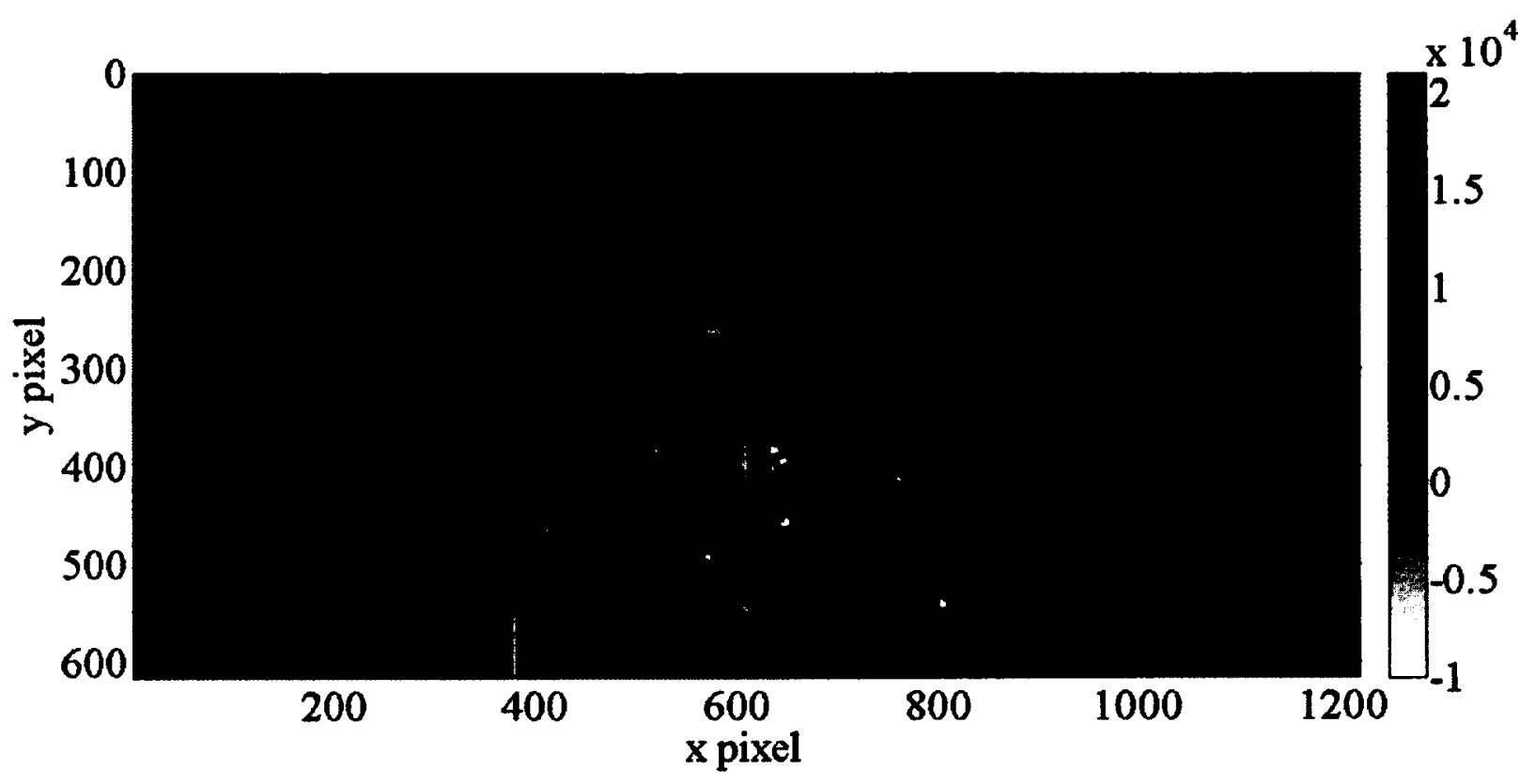

Figure 1.1: Filtered scatter pattern of $10 \mathrm{~mm}$ Lucite cuvette of ice.

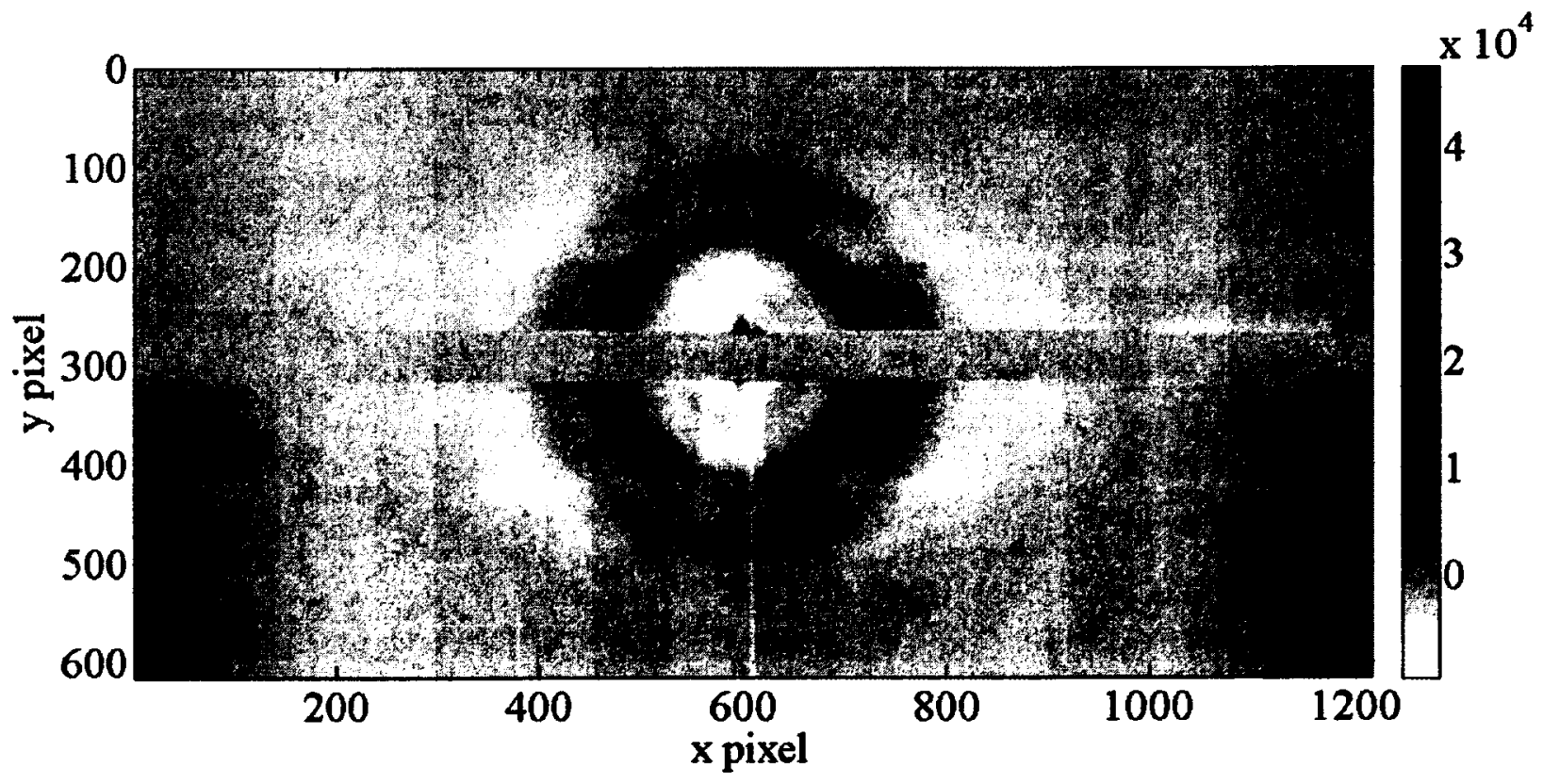

Figure 1.2: Filtered scatter pattern of $10 \mathrm{~mm}$ Lucite cuvette of water. 


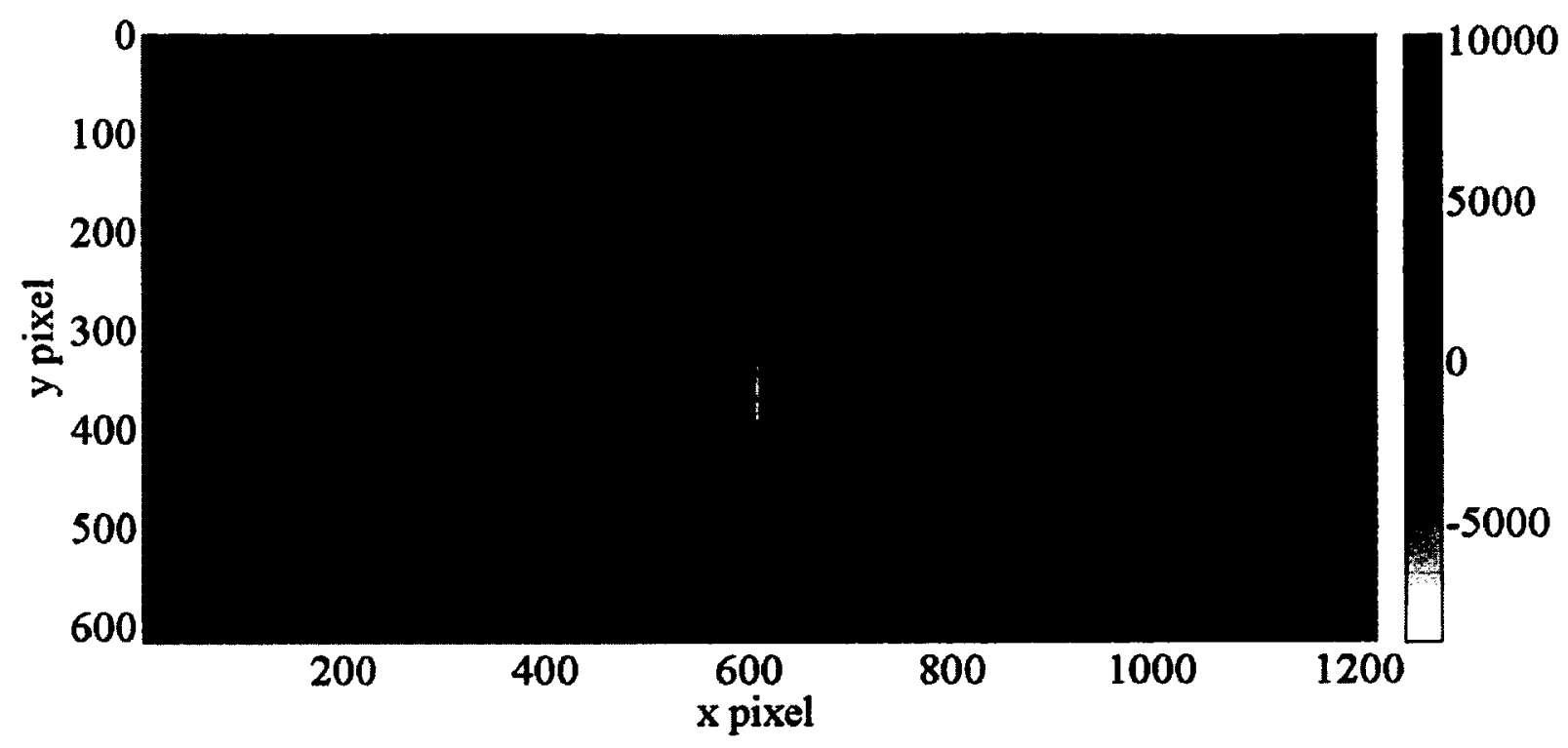

Figure 1.3: Filtered scatter pattern of Strontium Ranelate sachet. The extremely high intensity region near the centre of the scatter pattern is the tail-end of the primary beam.

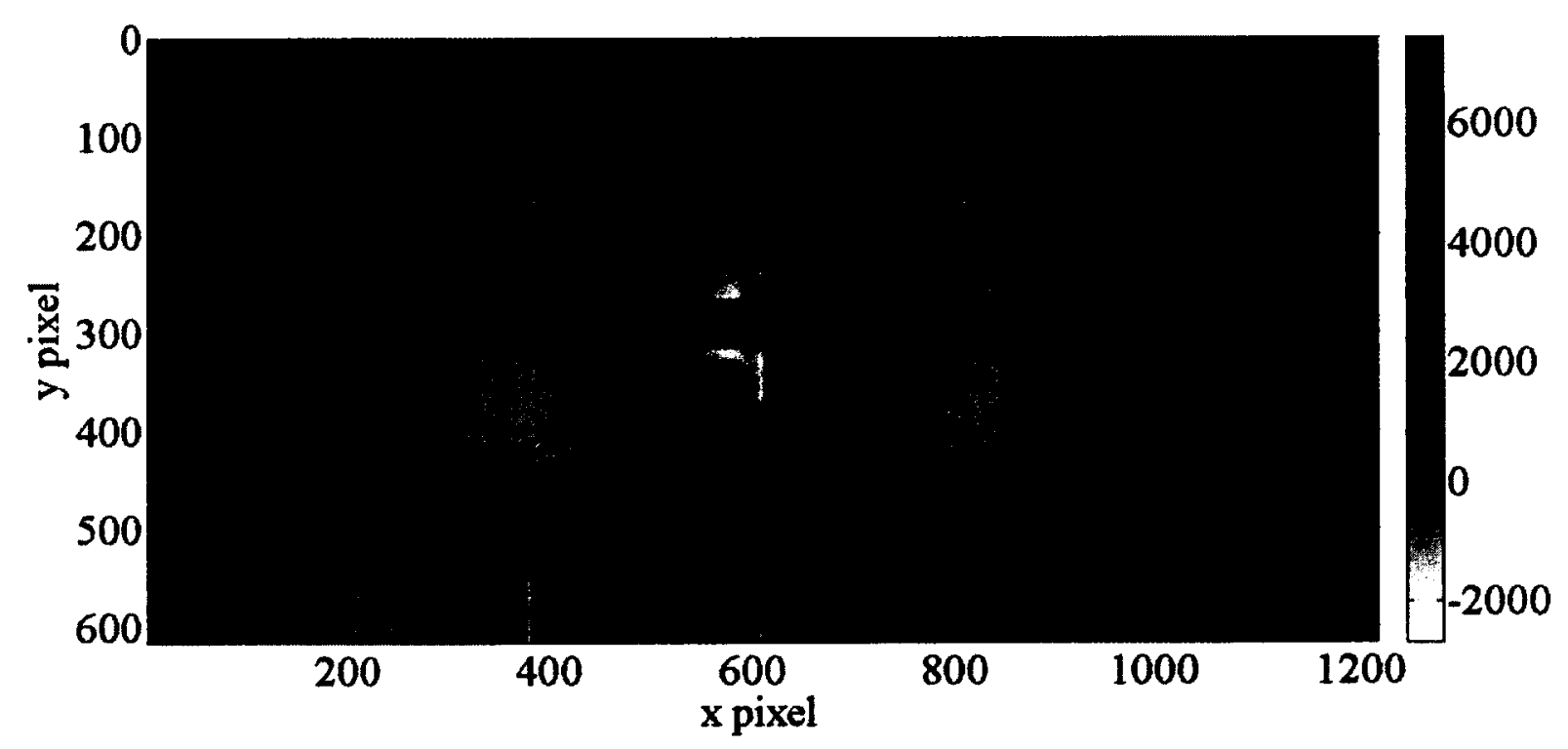

Figure 1.4: Filtered scatter pattern of a vitamin $C$ tablet dissolved in water in a $10 \mathrm{~mm}$ Lucite cuvette. 
Harding and co-workers (Refs. 8,9) first proposed the idea of using this type of scatter as a source of information for imaging and introduced a new cross-sectional imaging technique called $\mathrm{x}$-ray diffraction computed tomography. They used this technique to image a water/plastics CT phantom and showed that different contrasts can be obtained by changing the scatter angle, $\theta$. Investigators have looked at industrial and security applications for materials identification ${ }^{11}$ and detecting explosives. ${ }^{12,13}$ Philips set up a commercial system ${ }^{14}$ at Hamburg Airport utilizing coherent scatter. More recently Harding has applied $\mathrm{x}$-ray diffraction to determining effective density and atomic number of materials (tested on Lucite and water) ${ }^{15}$ and screening liquids and amorphous substances. ${ }^{16} \mathrm{X}$-ray diffraction can non-destructively extract several critical parameters about the material and allow it to be grouped into potentially dangerous categories, such as oxidizers or fuels.

Madden et al. ${ }^{17}$ developed a coherent $\mathrm{x}$-ray scattering CT system to enhance screening of explosives and other illegal materials. They showed that their system significantly reduced the rate of false alarms over traditional radiographic techniques. Grant et al. ${ }^{18}$ developed a microtomography system using a NaI(Tl)/PMT detector.

Westmore et al. ${ }^{19}$ demonstrated the potential of coherent scatter computed tomography by imaging a water-plastics CT phantom using an image intensifier-based system. They then developed a coherent-scatter cross section imaging system $^{20}$ by measuring the low-angle diffraction patterns of an object. Batchelar and Cunningham ${ }^{21}$ applied their system to material-specific imaging and Batchelar $e t a l .{ }^{22}$ investigated bonecomposition imaging. More recently Cunningham's group ${ }^{23}$ has set up a system utilizing balanced "Ross filters" to generate a pseudomonoenergetic beam to eliminate spectral 
blurring. They are investigating the applications of this system to kidney stone analysis. LeClair et $a .^{24}$ have applied $\mathrm{x}$-ray diffraction technology to the analysis of breast tissue biopsies. Cui et al. ${ }^{25}$ have developed diffraction CT to look at tissue scaffolds.

\subsection{Synchrotron light source}

A synchrotron is a particle accelerator which uses radiofrequency waves to accelerate and large magnetic fields to guide charged particles in a circular path. A synchrotron light source uses auxiliary devices, typically bend-magnets and insertion devices, to provide very useful electromagnetic radiation which can range from infrared to hard $\mathbf{x}$ rays. Our experimental work was performed at the BioMedical Imaging and Therapy Bend Magnet beamline (BMIT-BM) ${ }^{26}$ at the Canadian Light Source (CLS). The CLS is a third

generation 2.9 electron $\mathrm{GeV}$ synchrotron located in Saskatoon, Canada. ${ }^{27}$ A synchrotron was used because it provides high intensity (to reduce acquisition time) and monochromatic (to eliminate spectral blurring) radiation. Additionally the motorized sample stands were necessary in order to automate our system. We used the BMIT beamline because it is designed for this type of research, and can provide the desired energy along with the required detectors.

\subsection{Coherent scatter imaging research at Carleton University}

Most of the coherent scatter imaging system development to date has been of tomographic systems. Our approach is of projection imaging which holds the advantages of not requiring as many measurements in x-raying a volume and does not require beam access all the way around the object. 
LeClair and $\mathrm{Johns}^{28}$ developed a semianalytic model and compared an ideal conventional transmission system to an ideal coherent scatter system. Based on the scarce available data for tissue coherent scatter cross sections, they found that for the task of imaging white brain matter versus blood in a $150 \mathrm{~mm}$ thick water phantom, the maximum SNR, over all energies, for images using the forward scattered photons in the range $2^{\circ}$ $12^{\circ}$ is greater than the SNR for images based on primary photons for targets that are $\leq 23$ mm thick.

Our lab developed an energy-dispersive technique $e^{29,30}$ using an $x$-ray tube and high purity germanium spectrometer and we used $\mathrm{it}^{31}$ to measure the coherent scatter cross sections for water and a variety of tissues and plastics

Scatter projection imaging work by Nisar and Johns ${ }^{32}$ demonstrated the concept of multiple pencil beams in an hexagonal array and used computed radiography to record the scatter patterns of plastics and tissues in air and in a water tank.

Initial work at the Canadian Light Source synchrotron by Johns et al ${ }^{33}$ utilized a monoenergetic fan beam and captured the scatter patterns using an Agfa computed radiography plate. The overlap of information caused by the fan beam, however, motivated the focus on pencil beams in the present work.

\subsection{Motivation and objectives}

The main objective of this research was to develop a system at the Canadian Light Source to produce coherent scatter images using multiple $\mathrm{x}$-ray pencil beams. This work was done as a proof of principle and to compare scatter images to conventional images since previous theoretical work ${ }^{28}$ predicted coherent scatter to have higher contrast and signal 
to noise. Conventional $\mathrm{x}$-ray imaging treats scatter as a nuisance and does not utilize any of this free useful information. Multiple radiation pencil beams were used to reduce acquisition time and improve the utilization of the source output. The main application of this work is medical imaging. In particular this work would be best applied to procedures which require high soft-tissue contrast with thin samples, such as mammography. Thus tissue phantoms are prevalent throughout all experiments, but other applications include non-destructive testing and screening. Ultimately this technology will be transferred to standard hospital x-ray tubes.

In this work various coherent scatter projection images of tissue, water and plastic phantoms were made. This work shows the benefits and drawbacks of the technique. The details of the experimental setup at the Canadian Light Source synchrotron are described in Chapter 3.

\subsection{Statement of contribution}

My supervisor and I planned and executed all four of the experiments at the CLS, with support from the technical staff there. The collimator shown in this work was designed by me and built by the Science Technology Centre at Carleton University. I developed and implemented both of the disentangling algorithms along with the code to filter scatter patterns, convert both the primary and scatter data to images, and the code to display images in a hexagonal lattice. All the code listed in the Appendix is my original work. I drafted the publications and am lead author on them.

Portions of this work were presented at the 2011 joint American Association of Physicists in Medicine/Canadian Organization of Medical Physicists meeting, ${ }^{34}$ in the 
form of a poster presentation. An oral presentation was given at the 2012 SPIE: Defense, Security and Sensing conference and a proceedings published. ${ }^{35}$ A paper reporting our most recent work has been submitted to the journal Review of Scientific Instruments.

\subsection{Outline of thesis}

Chapter 2 explains the theory of coherent scattering and the physics of digital flat panel detectors. Chapter 3 explains the experimental setup and method for creating both primary and scatter images. In Chapter 4 the two main disentangling algorithms developed for this project are described and tested. Chapter 5 gives results, including many single and multi-beam coherent and primary images, comparison of them, and a comparison of images made from both single and multi-beams. Chapter 6 gives conclusions and future work. An Appendix is included at the end which contains the MATLAB (MathWorks, Natick, Massachusetts) code used to process the data and generate images. 


\section{Chapter 2: Theory}

\subsection{Coherent scatter}

Coherent scatter is one of the three interactions a photon can undergo in the diagnostic $x$ ray energy range. Alternatively a photon can Compton scatter off an electron, where its energy after collision is given by

$$
\mathrm{E}^{\prime}=\frac{E}{1+\frac{E}{m_{e} c^{2}}(1-\cos \theta)},
$$

where $E$ is the energy of the photon, $m_{e}$ is the mass of an electron, $c$ is the speed of light and $\theta$ is the angle between incoming and outgoing photons. A photon can also be absorbed by an electron causing the electron to be emitted from the atom, resulting in subsequent fluorescent $\mathrm{x}$ rays or Auger electron emission. This process is known as the photoelectric effect.

Figure 2.1 is a log-log plot of photon cross sections versus energy for water, ${ }^{36}$ for 10 to $500 \mathrm{keV}$. This thesis focuses on utilizing information from coherently scattered photons. Coherent scatter is a phenomenon where the oscillating electric field of electromagnetic radiation causes a momentary vibration of the electron cloud of the atom or molecule. Each electron/nucleus combination emits classical dipole radiation. There is an interference effect between the emitted radiation from each electron. The constructive interference becomes the scatter patterns we measure. This process happens nearly instantaneously and the emitted energy is very nearly equal to the absorbed energy. By conservation of momentum the emitted photon is actually of very slightly less energy 
than the impinging photon (unless the direction of the photon is unaffected), as some momentum (and thus energy) must be transferred to the recoiling atom or molecule. However, since the atom or molecule is very massive the energy difference is negligible. Figure 2.2 is a diagram illustrating the process of coherent scatter. In the "before" panel a photon of wavelength $\lambda$ impinges on an atom. In the "intermediate" panel the electrons/nucleus vibrate and in the "after" panel a photon is emitted of (nearly) the same wavelength as the impinging photon but in a different direction. The cross section of coherent scatter is determined by the individual spatial locations of electrons, which are defined by the atomic or molecular structure of the material. Coherent scattering is peaked at very small forward angles. Thus despite its relatively small cross total section (as seen in Figure 2.1), in the range of $0-10^{\circ}$ coherent scatter dominates. ${ }^{2}$

The coherent scattering cross section per electron per steradian ${ }^{37}$ for unpolarized radiation is:

$$
\frac{d_{e} \sigma_{c o h}}{d \Omega}=\frac{r_{0}^{2}}{2}\left(1+\cos ^{2} \theta\right) \frac{F^{2}(x, Z)}{Z},
$$

where $r_{0}$ is the classical electron radius, $F(x, Z)$ is the coherent scatter form factor, $Z$ is the number of electrons in the stoichiometric unit considered and the subscript $e$ is used to denote that it is per electron. The quantity $x$ is given by

$$
x=\frac{1}{\lambda} \sin \left(\frac{\theta}{2}\right)=\frac{E}{h c} \sin \left(\frac{\theta}{2}\right),
$$

where $\lambda$ is the photon wavelength, $E$ is the energy, $h$ is Planck's constant and $c$ is the speed of light. Since the change in momentum is $\Delta p=2 h x$ the quantity $x$ is termed the momentum transfer argument. Figure 2.3 is a plot of differential cross section versus 
scattering angle ${ }^{31}$ for water showing the forward-peaked nature of coherent scatter for $33.17 \mathrm{keV}$ (the energy used in our experiment).

The form factors must be known to calculate the ideal angular range which will maximize contrast between two different materials. In general it is very difficult to calculate the coherent scatter cross section theoretically from quantum mechanics. The coherent scatter form factors can be measured using crystal diffractometers but there are difficulties applying this technology to amorphous materials. ${ }^{38}$ Consequently, prior research in our lab developed an energy-dispersive technique ${ }^{29,30}$ using an $\mathrm{x}$-ray tube and high purity germanium spectrometer and we used $\mathrm{it}^{31}$ to measure $F(x, Z)$ for water and a variety of tissues and plastics. Some of these data have been used as input to the work reported in this thesis.

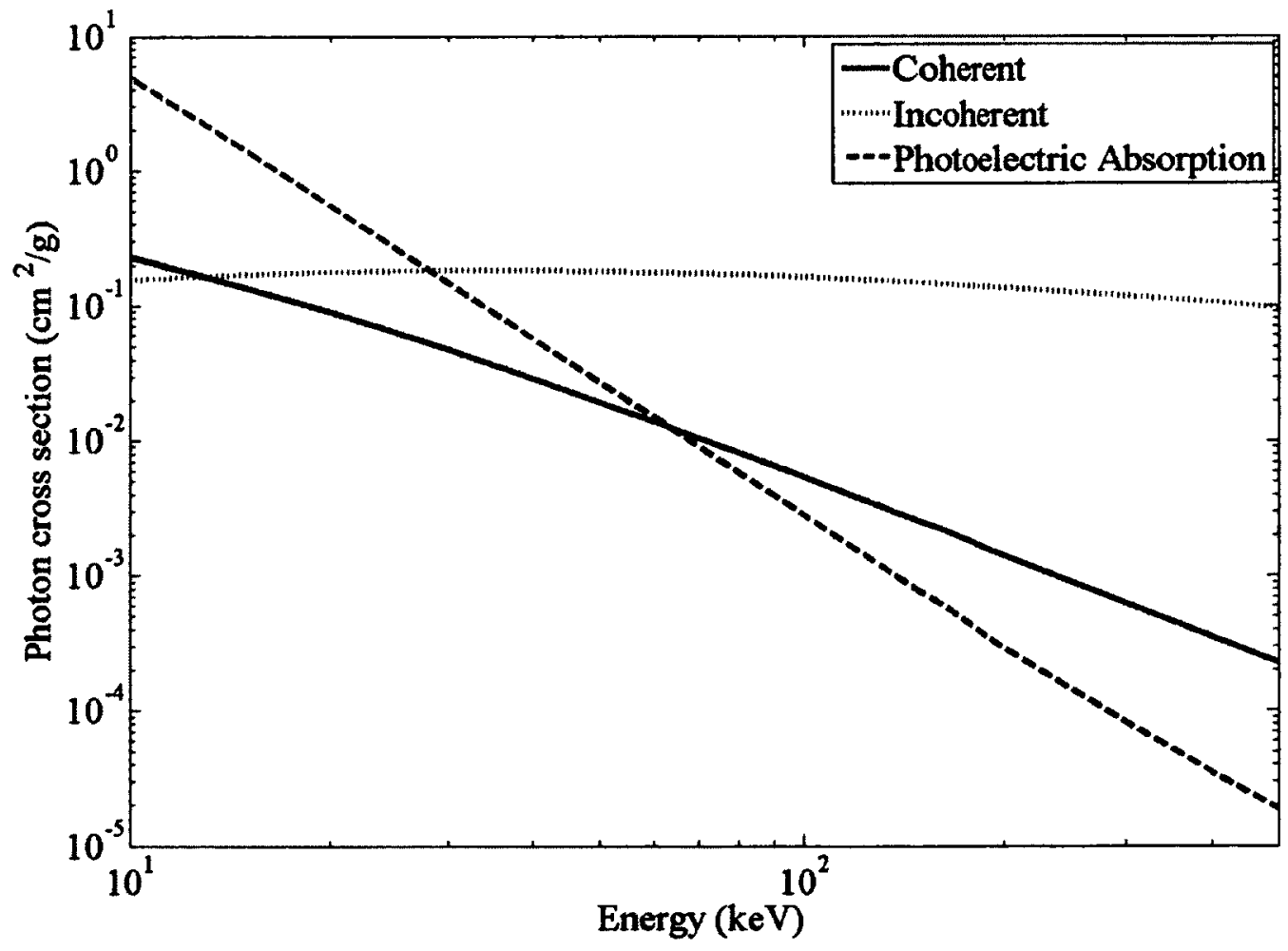

Figure 2.1: $\log -\log$ plot of photon interaction cross sections of water versus energy from 10 to $500 \mathrm{keV}$ (data from Ref. 36). 


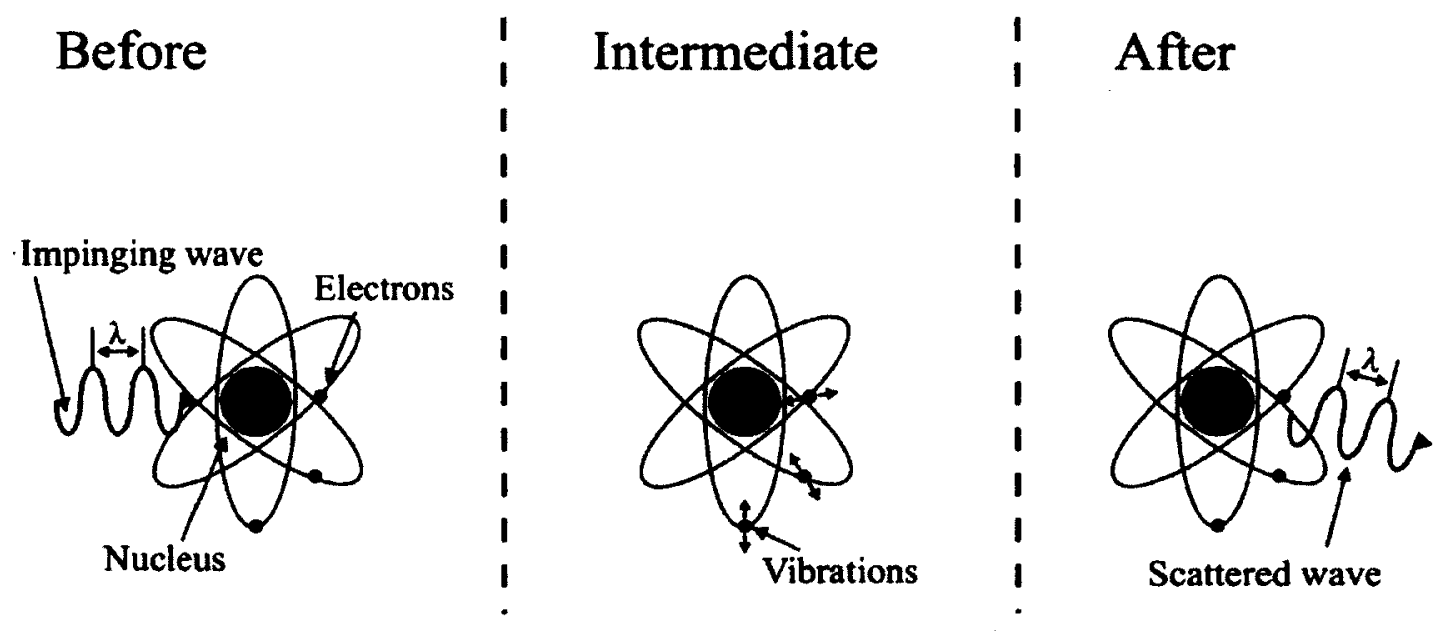

Figure 2.2: Illustration of the process of coherent scatter.

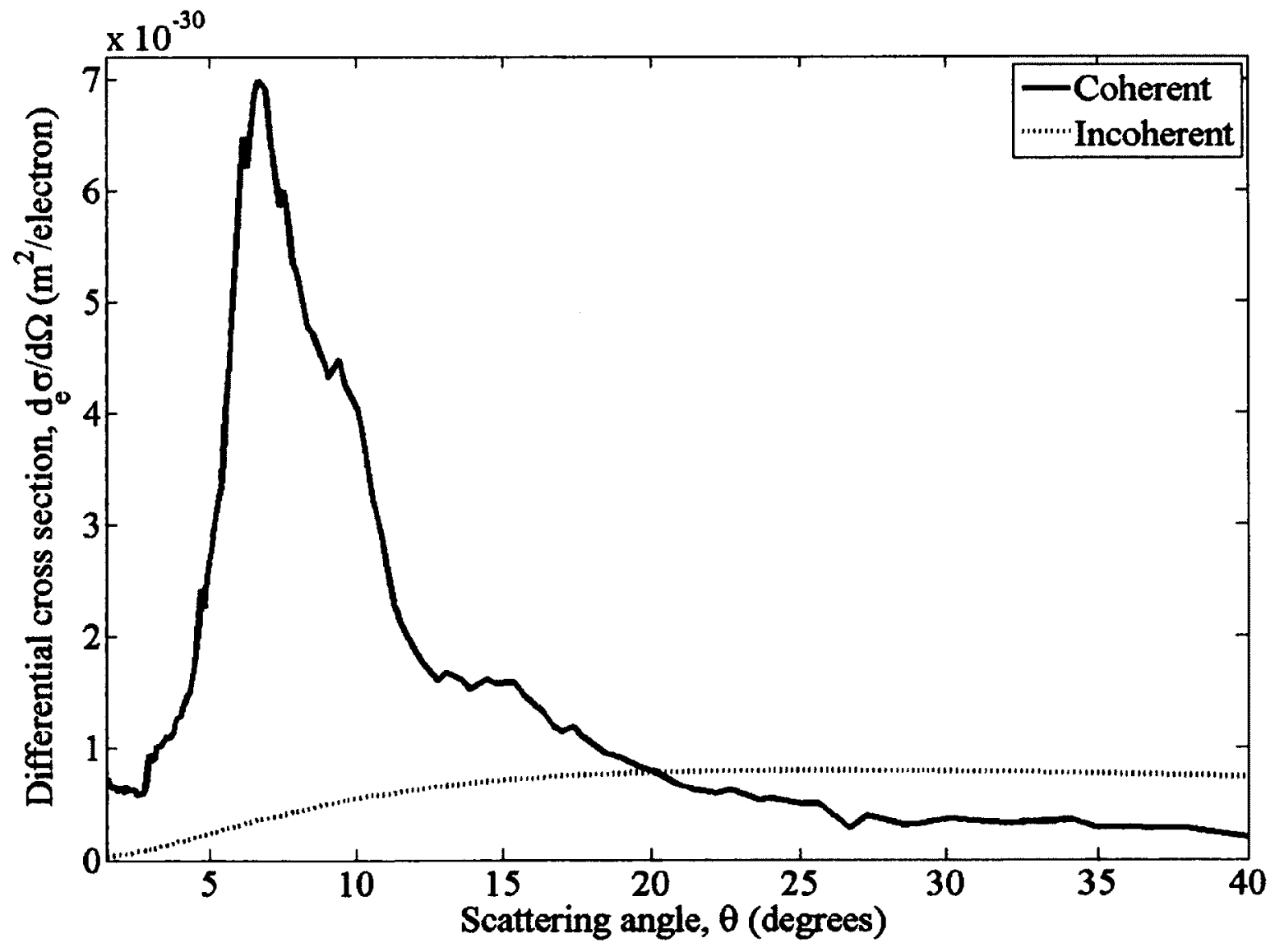

Figure 2.3: Plot of differential cross section per electron versus scattering angle for water at $33.17 \mathrm{keV}$ (data from Ref. 31 ). 


\subsection{Flat panel digital x-ray detector}

There are two main types of flat panel digital $\mathrm{x}$-ray detectors: direct conversion detectors and indirect-conversion detectors. Direct conversion detectors utilize an x-ray photoconductor (typically amorphous selenium) that directly converts x-ray photons into an electric charge. The Hamamatsu C9252DK-14 detector used in our work uses an indirect-conversion method. For these detectors a scintillating material (in our case crystalline CsI) is directly deposited onto a two-dimensional photodiode array. X rays incident on the scintillating material are converted to fluorescence which enter the twodimensional photodiode array. A charge accumulates in each pixel proportional to the light (and thus $\mathrm{x}$-ray) intensity. The charge is then read out as a voltage. 


\section{Chapter 3: Method}

\subsection{Basic concept}

Figure 3.1 is a flow diagram of the method to create scatter images for a single beam. The only difference for multiple beams is the collimator between the source and the sample and a disentangling algorithm which replaces the averaging over concentric circles. The double-ended arrows indicate the mechanical motion to raster scan the sample through the beam to acquire a pixel at each location. The image generation is done after all the data are collected. For single-beam images each scatter pattern corresponds to one radial profile which is integrated over a specified range to generate one pixel in the scatter image.

\subsection{Experimental setup}

Experimental work was done at the BioMedical Imaging and Therapy Bend Magnet beamline ${ }^{26}$ (BMIT-BM) at the Canadian Light Source synchrotron (CLS), in Saskatoon, Saskatchewan. The CLS is a $2.9 \mathrm{GeV}$ third generation synchrotron. ${ }^{27}$ The BMIT-BM can provide a beam in the energy range of $15-40 \mathrm{keV}$ with a photon brightness of $1.5 \times 10^{11}$ photons $/\left(\mathrm{s} \mathrm{mr}^{2} 0.1 \% \mathrm{bW} \mathrm{mA}\right)$ at $10 \mathrm{keV}$ where the "mA" refers to the $2.9 \mathrm{GeV}$ storage ring electron current which is typically $150-250 \mathrm{~mA}$. This beamline can produce a beam size of up to $240 \mathrm{~mm}$ horizontal and $7 \mathrm{~mm}$ vertical at $23 \mathrm{~m}$ from the source. The distance is large and the beam can therefore be treated as parallel. In a traditional hospital setup the distance is approximately $1 \mathrm{~m}$ and the beam is not parallel. 

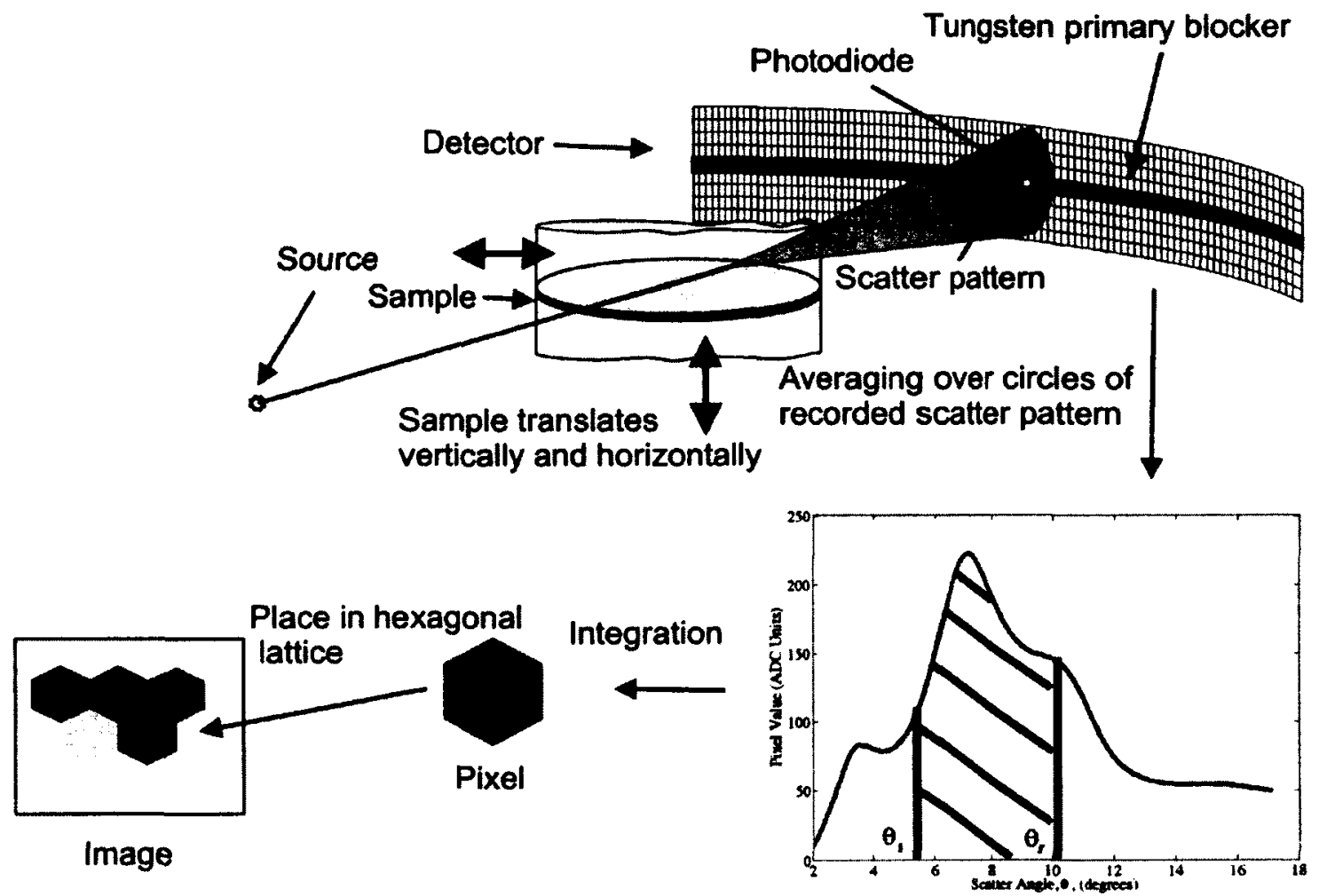

Radial profile

Figure 3.1: Flow diagram of method to create single beam scatter images.

For this work a bent Silicon- $(3,1,1)$ crystal Laue monochromator was used to generate a monochromatic beam at $33.17 \mathrm{keV}$ (with an energy resolution of $\Delta E=200 \mathrm{eV}$ ) to eliminate unwanted spectral blurring. Due to the width of the crystal, however, the beam can only be a maximum of $80 \mathrm{~mm}$ wide. The scatter patterns were measured by a flat panel digital x-ray detector (C9252DK-14, Hamamatsu Photonics, Hamamatsu, Japan) (pixel size $200 \mu \mathrm{m}$ by $200 \mu \mathrm{m}, 1216$ by 616 pixels).

The target was mounted on a sample stage controlled by a servo motor (HV23302-FL, Parker Hannifin, Cleveland, Ohio) with a positional precision of $90 \mu \mathrm{m}$. A custom built collimator consisting of $20.2 \mathrm{~mm} \times 6.3 \mathrm{~mm} \times 3.3 \mathrm{~mm} 56 \%$ tungsten $/ 44 \%$ copper 
slugs (Eagle Alloys Corporation, Talbott, Tennessee) with $1.5 \mathrm{~mm}$ aluminum spacers was designed to segment an impinging fan beam into multiple pencil beams $1.5 \mathrm{~mm}$ wide by the beam thickness in height ( 0.2 to $0.6 \mathrm{~mm}$ depending on the lateral position). This multiplexing is necessary in order to decrease acquisition time by a factor equal to the number of beams. Figure 3.2 and Figure 3.3 are the schematics for the collimator, and Figure 3.4 is a photograph of the collimator set up for two beams spaced approximately $24 \mathrm{~mm}$ apart. Figure 3.5 is a photograph showing one tungsten-copper stopper and one aluminum beam spacer beside the base of the collimator. The size of the pencil beams is defined by the width of the aluminum spacer. The collimator was built by the Science Technology Centre at Carleton University. In practice lead was added above and below the collimator to block unwanted scatter from other objects inside the room.

\section{Top View}

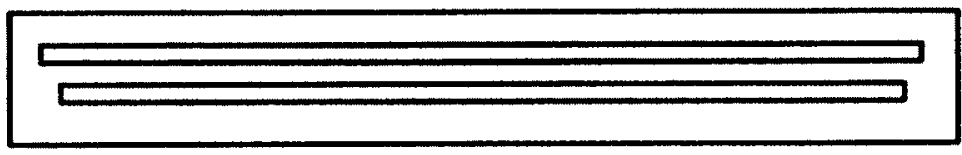

Side Views

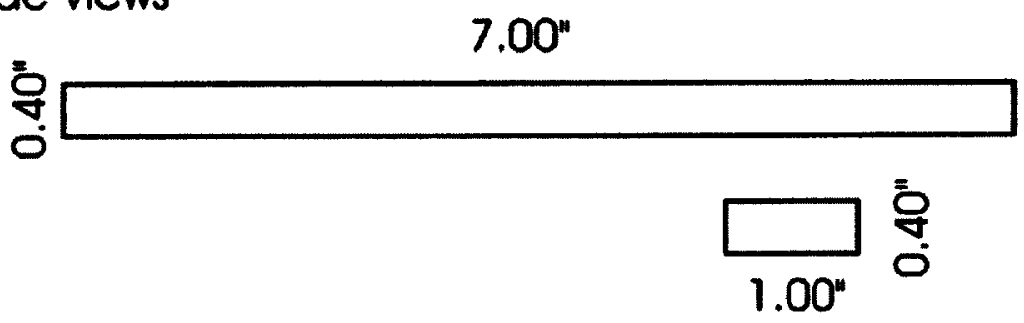

Angled View

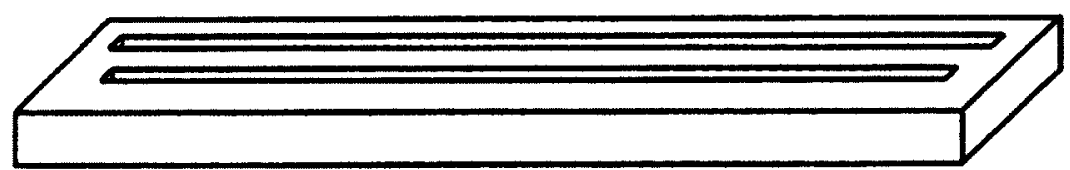

Figure 3.2: Schematic of custom collimator base without tungsten stoppers in the channels. Multiple view angles are displayed. The collimator base is $(10.2 \mathrm{~mm}$ by 25.4 $\mathrm{mm}$ by $177.8 \mathrm{~mm}$ ). 


\section{Angled View}

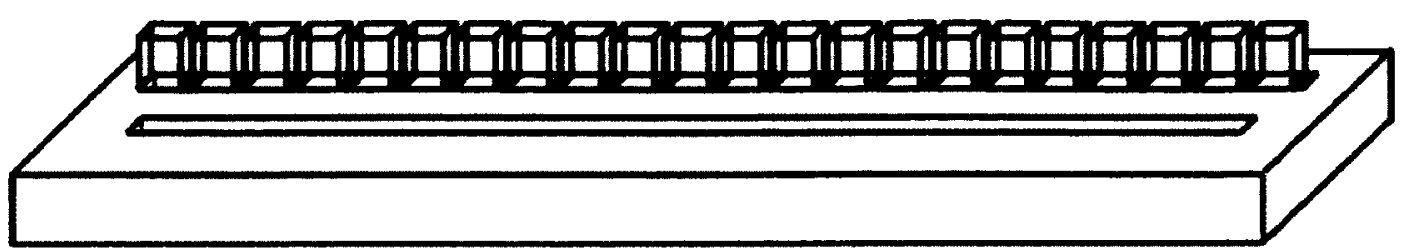

Figure 3.3: Concept diagram of collimator with one full row of tungsten stoppers and one empty one. This would convert a fan beam into 21 pencil beams of approximately 1.5 $\mathrm{mm}$ width and $8 \mathrm{~mm}$ spacing, centre to centre. The fan beam from the monochromator is only about $80 \mathrm{~mm}$ wide and therefore can only support a maximum of 10 beams.

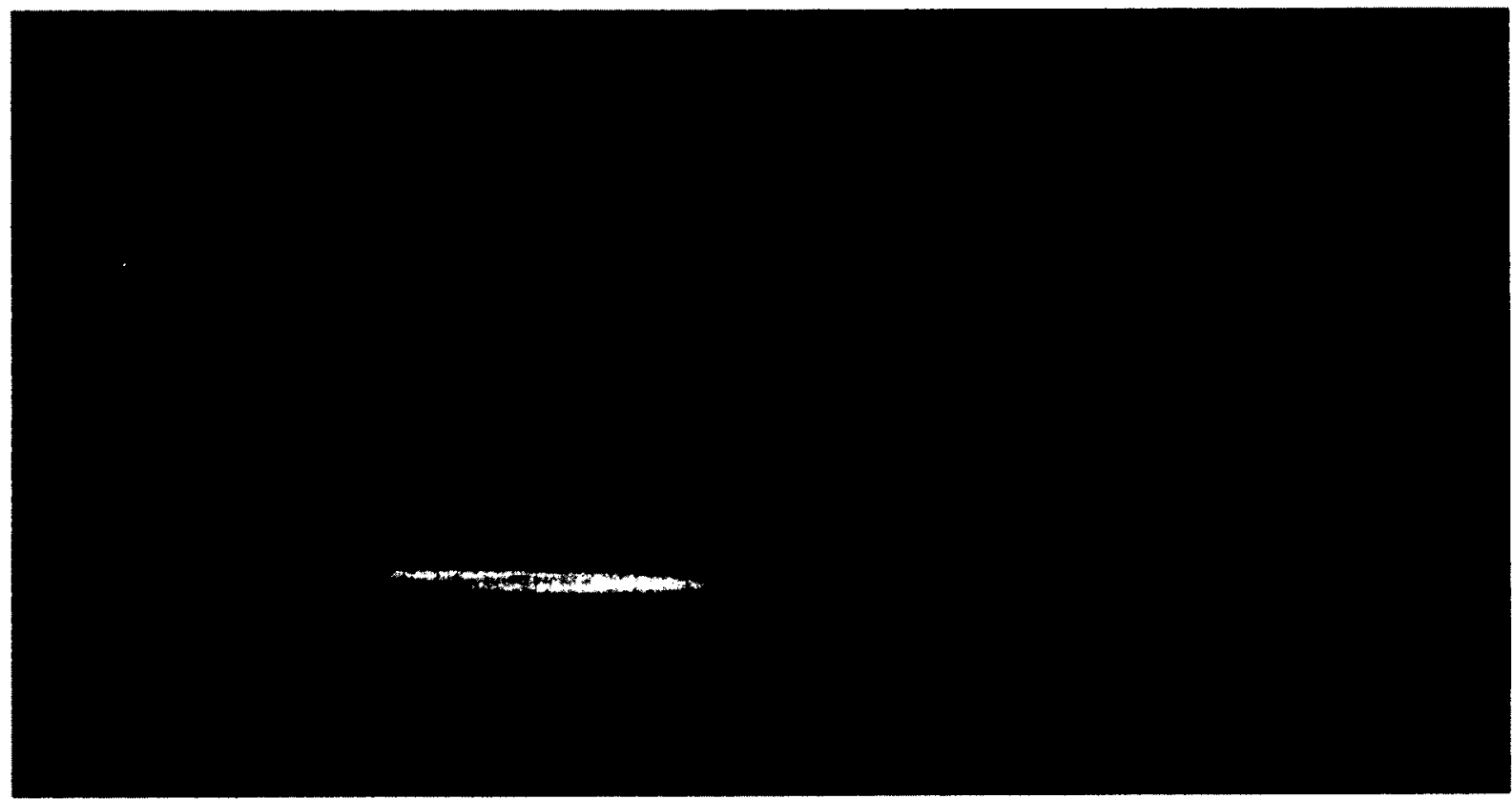

Figure 3.4: Photograph of customizable collimator to convert fan beam into multiple pencil beams. Tungsten-copper stoppers can be easily removed to increase the number of beams or change the spacing between beams. The front row of stoppers segments a fan beam into multiple pencil beams and the second row selects which ones will be used. This setup is for two beams spaced approximately $24 \mathrm{~mm}$ apart. 


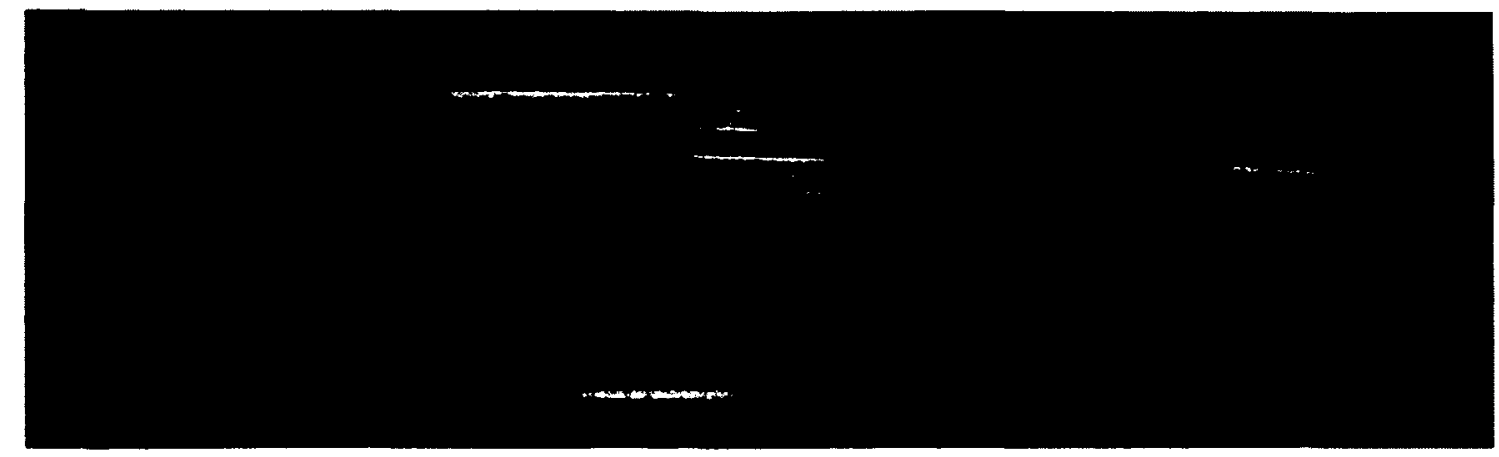

Figure 3.5: Photograph of (from left to right) aluminum spacer, tungsten stopper and collimator base.

The multiple primary beams travel through the sample and are stopped by a tungsten bar ( $9 \mathrm{~mm}$ wide by $11 \mathrm{~mm}$ thick in the beam direction) before reaching the flat panel detector. Thus only scattered $x$ rays reach the detector and at low angles these are mostly coherent scatter. By using multiple beams we measure a superposition of scatter patterns on the detector.

The beam thicknesses were measured by placing a piece of Gafchromic film on the tungsten beam-stopper and exposing it for $5 \mathrm{~min}$. The beam sizes were then measured using a low-power travelling microscope and camera. The dimensions of the five beams were found to be $1.5 \mathrm{~mm}$ by 0.2 to $0.6 \mathrm{~mm}$ in the horizontal and vertical directions, respectively. The variation is due to imperfections in the structure of the crystal or its bending. Figure 3.6 is a photograph of the Gafchromic film of a five beam exposure.

Figure 3.6: Photograph of Gafchromic film of five beams placed over grid paper. The large divisions on the grid are $10 \mathrm{~mm}$ and the smallest division is $1 \mathrm{~mm}$. 
Primary and scatter are difficult to measure with the same detector because although their total photon numbers are similar the primary photon numbers per unit area are orders of magnitude greater. The data for the primary images were recorded using various photodiodes. Table 3.1 indicates which photodiode and connecting cable was used for the different experiments at the Canadian Light Source.

Table 3.1: Types of photodiodes and cables used at the CLS.

\begin{tabular}{|l|l|l|l|}
\hline \multicolumn{1}{|c|}{$\begin{array}{l}\text { Date of } \\
\text { experiment }\end{array}$} & \multicolumn{1}{|c|}{ Photodiode used } & $\begin{array}{l}\text { Maximum } \\
\text { number of } \\
\text { photodiodes }\end{array}$ & $\begin{array}{l}\text { Type of coaxial } \\
\text { cable and } \\
\text { connector }\end{array}$ \\
\hline $\begin{array}{l}\text { August } \\
2010\end{array}$ & N/A & 0 & N/A \\
\hline June 2011 & $\begin{array}{l}\text { IRD AXUV4BST (International } \\
\text { Radiation Detectors Inc., Newbury } \\
\text { Park, California) }\end{array}$ & 1 & $\begin{array}{l}\text { Standard coaxial, } \\
\text { BNC }\end{array}$ \\
\hline $\begin{array}{l}\text { December } \\
2011\end{array}$ & $\begin{array}{l}\text { OSRAM SFH206K (OSRAM Opto } \\
\text { Semiconductors GmbH, Regensburg, } \\
\text { Germany) }\end{array}$ & 3 & $\begin{array}{l}\text { Standard coaxial, } \\
\text { BNC }\end{array}$ \\
\hline May 2012 & $\begin{array}{l}\text { OSRAM BWP 34 (OSRAM Opto } \\
\text { Semiconductors GmbH, Regensburg, } \\
\text { Germany) }\end{array}$ & 5 & $\begin{array}{l}\text { UT-047-AL-TP } \\
\text { (Micro-Coax, } \\
\text { Pottstown, } \\
\text { Pennsylvania), } \\
\text { SMA to BNC }\end{array}$ \\
\hline
\end{tabular}

The photodiodes were positioned by first exposing a piece of Gafchromic film attached to the primary beam stopper. The photodiodes were then taped onto the bar to match the actual spacing of the beams. Figure 3.7 is a photograph of the five OSRAM BWP 34 photodiodes mounted on the tungsten bar. The photodiodes were connected to current preamplifiers (SR570 - Low noise current preamplifier, Stanford Research Systems, Inc., Sunnyvale, California) and the signals digitized. 


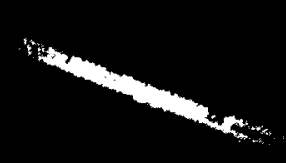

Figure 3.7: Five OSRAM BWP 34 photodiodes mounted on tungsten primary beam stopper.

Figure 3.8 is a concept diagram of acquisition of multiple scatter patterns on a single detector. It is a representation of the actual experimental setup of the CLS. The six main experimental pieces (source, collimator, sample, photodiodes, tungsten bar and detector) are shown. Figure 3.9 is a photograph of the experimental setup for multiple beams. It is taken in the general direction of the beam. Individual components of the setup have been labeled. The sample would raster scan through the stationary beam to image the desired area. At each individual sample location we acquire the scatter pattern (used to create the scatter image) and the photodiode signals (used to create the primary image). 


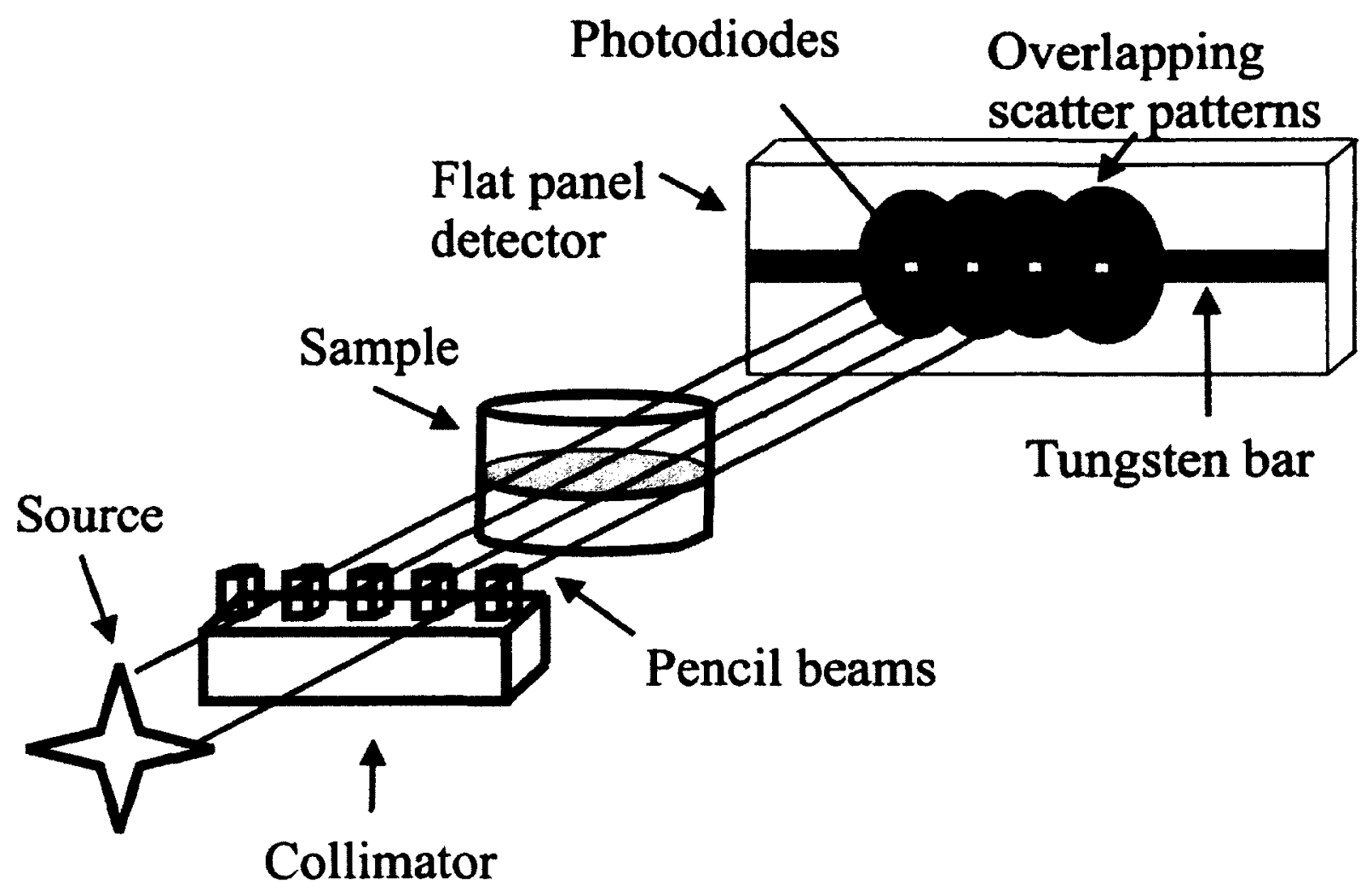

Figure 3.8: Concept diagram for multi-beam experimental setup. This is set up for four beams for illustration.

For multiple beams each beam only exposes a vertical strip of the total image. Figure 3.10 shows the acquisition scheme for two beams in a hexagonal pixel lattice, which can easily be generalized to $n$ beams. The white shaded area is used to illustrate the area covered by beam 1, and the gray is used to illustrate the area covered by beam 2 . The numbers indicate the order in which the pixels are measured. For this illustration there are two beams so two pixels are being measured (by individual photodiodes) simultaneously. For this diagram each beam only covers half of the entire sample. For $n$ beams each beam would cover $1 / n$ of the sample. 


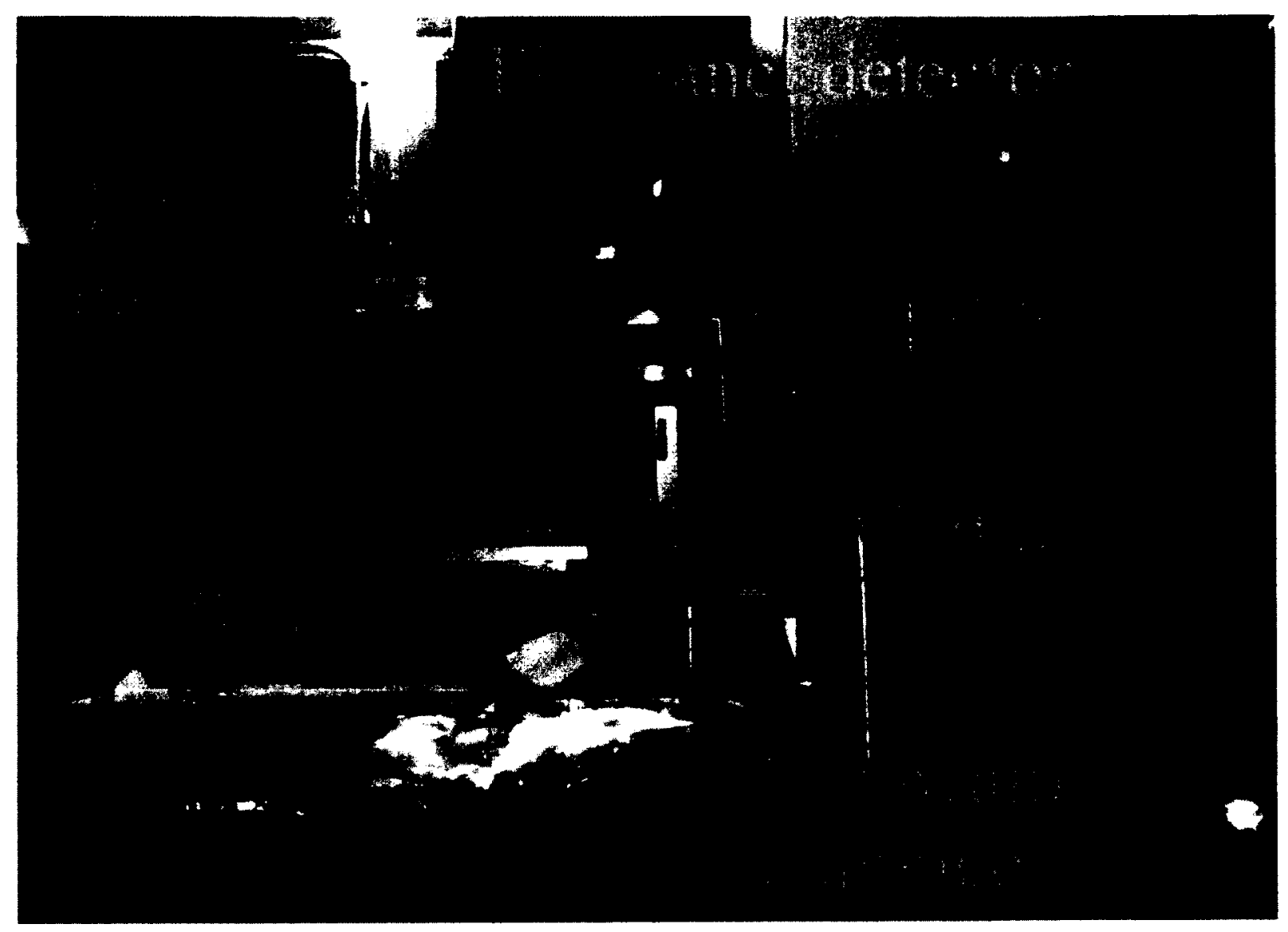

Figure 3.9: Photograph of the experimental setup for multiple beam scatter pattern acquisition at the CLS. This photograph is taken in the general direction of the beam. 


\section{Beam $1 \quad$ Beam 2}

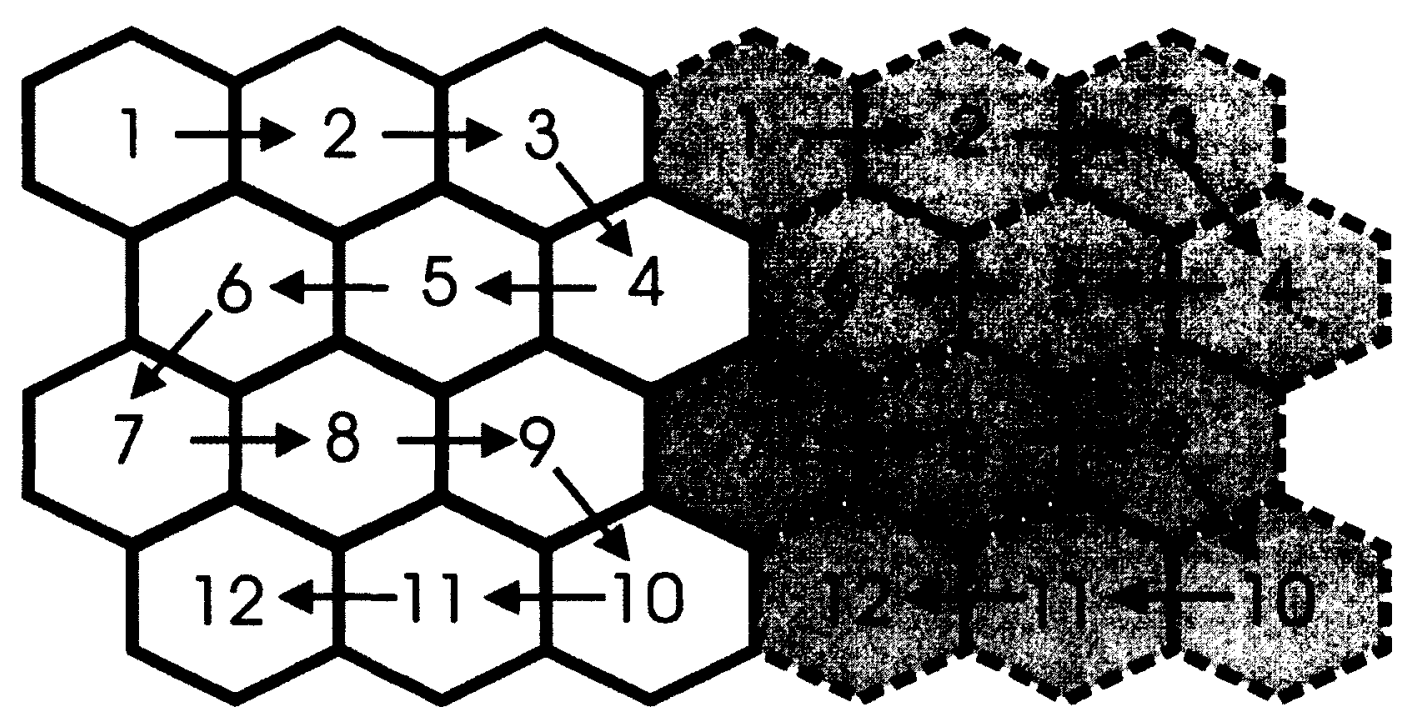

Figure 3.10: Acquisition of data scheme illustrated for two beams. Both beams start at the top left of their scanning region and then raster scan in the order indicated in the diagram.

\subsection{Creating the primary image}

The beam separation and beam size are defined by the dimensions of the tungsten stoppers and aluminum spacers. The separation chosen was $32.0 \mathrm{~mm}$ when two beams were used and $16.0 \mathrm{~mm}$ for three beams and up. The pixel value is found by averaging the photodiode voltage signal (typically on the order of a Volt) over the entire exposure duration. The pixel is then placed into the primary image. Since there are multiple different photodiodes with different responses and multiple beams with different sizes and intensities a normalization was done. Otherwise a line between the two beams is evident in the primary image. The normalization simply computed the average of the column directly to the left of the boundary and divided it by the average directly to the right of the boundary to obtain a single value. The right portion of the image is then 
multiplied by this value. This is done for each individual boundary, starting with the leftmost first.

Figure 3.11 is the primary image of a standard line pair resolution pattern. The resolution was found to be less than 0.6 line pairs per $\mathrm{mm}$, which is the minimum value of the bar pattern. This is because our pencil beam spacing $(1 \mathrm{~mm})$ is greater than the Nyquist sampling step size.

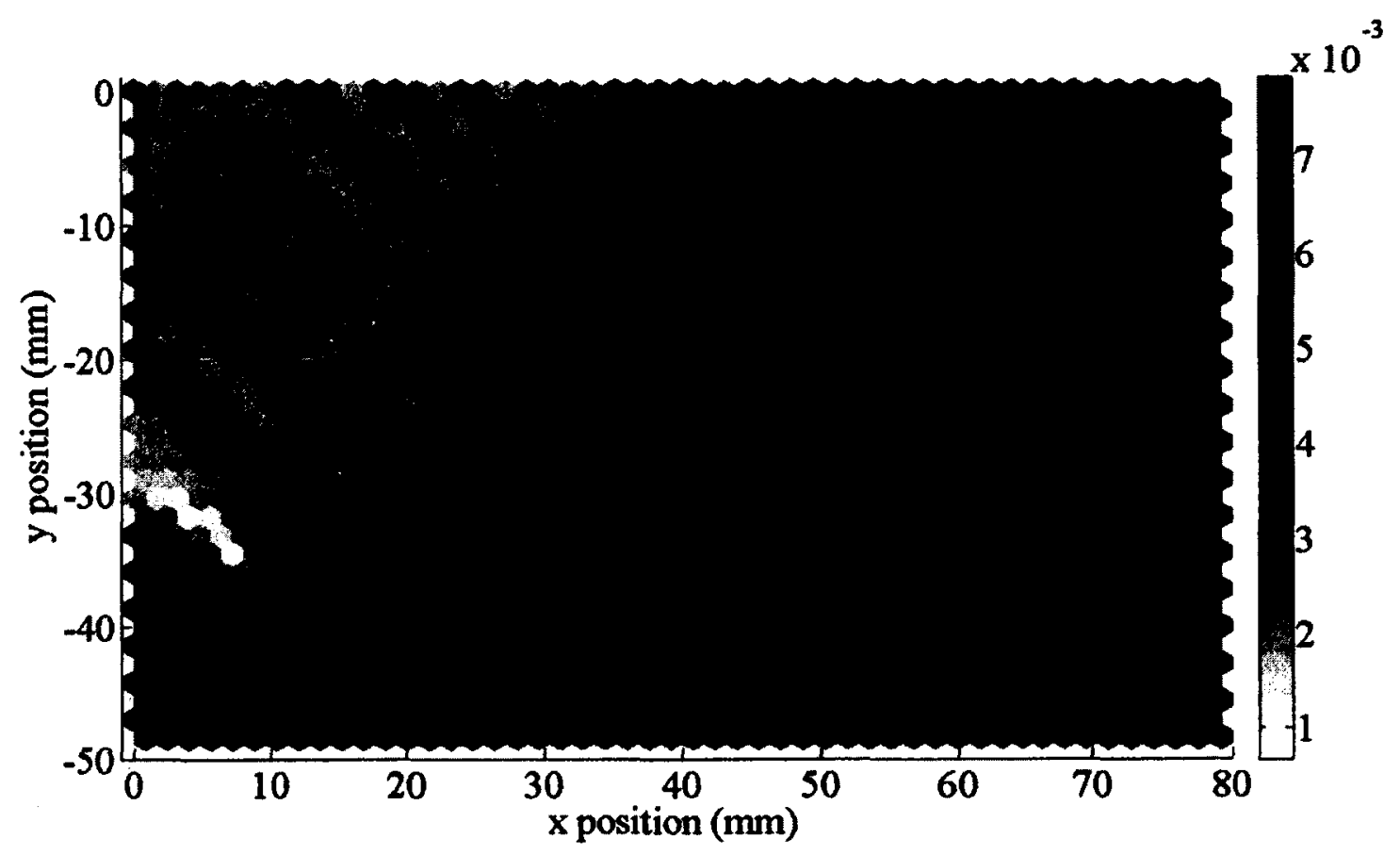

Figure 3.11: Primary image of a standard line pair resolution pattern. The pattern consists of lines of lead separated by varying distances. The largest group is 0.6 line pairs per $\mathrm{mm}$ which are not distinguishable with our sampling step size.

\subsection{Creating the scatter image}

The scatter image is acquired simultaneous to the primary. The raw data from the detector must be filtered prior to processing; the details of this are given in Section 4.2. We designate the result from the filtering as $S$. We used a uniform plastic bar as a 
calibration and test object (Figure 3.12). Figure 3.13 is the filtered scatter pattern of the uniform plastic bar with three beams. It should be noted that the bright region of $\mathrm{x}$ pixel $560-580, y$ pixel $1-260$ is due to a photodiode coaxial cable blocking a portion of the scatter.

Figure 3.12: Uniform plastic bar used as test object to evaluate individual algorithms. The bar is smooth and has dimensions of $10 \mathrm{~mm} \times 10 \mathrm{~mm} \times 180 \mathrm{~mm}$.

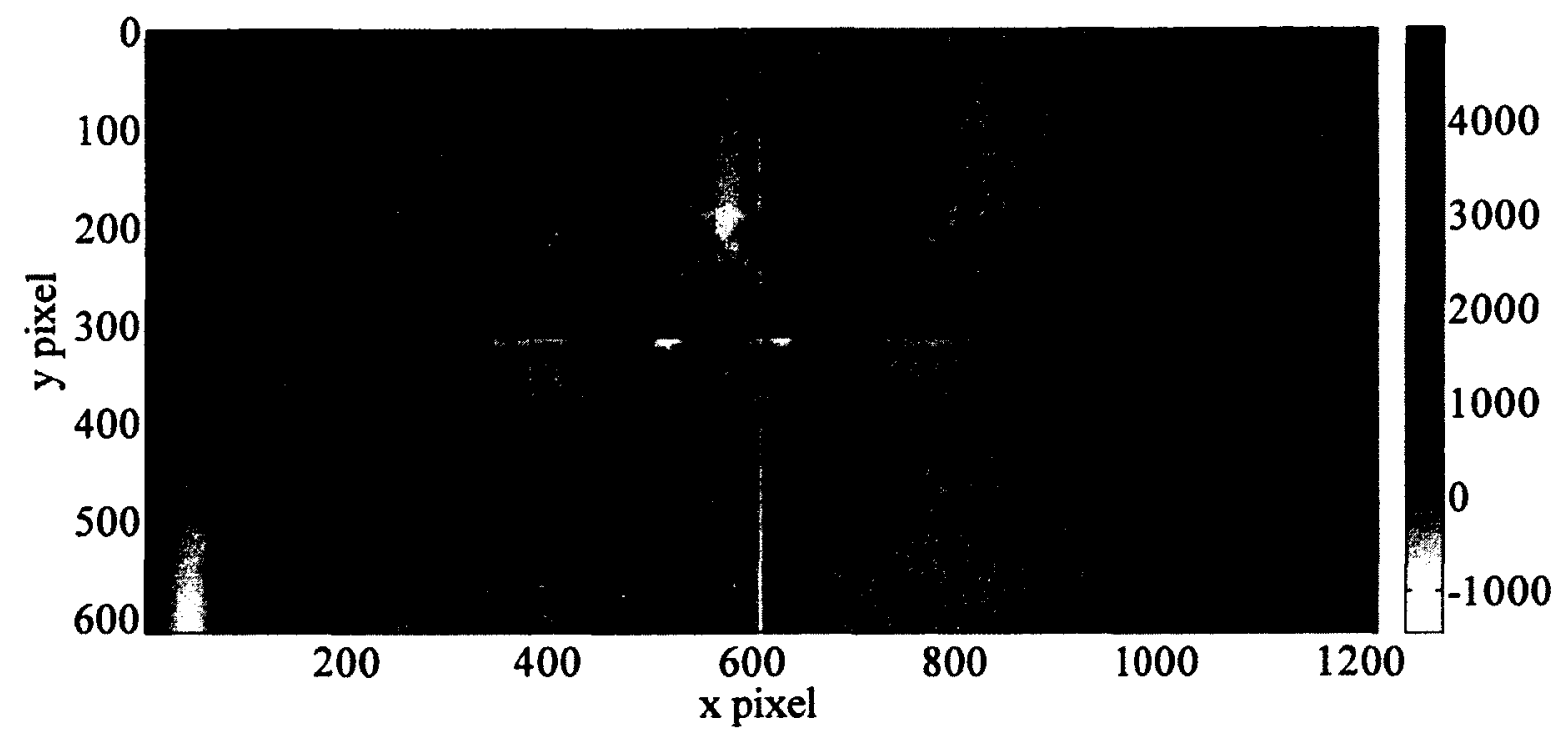

Figure 3.13: Scatter pattern of a uniform plastic bar that has been median-filtered, highpass filtered and had background subtracted. Three individual pencil beams were used.

These filtered data are then run through a maximum-likelihood expectation maximization (MLEM) algorithm to obtain the radial profile, $r$, for the path of each of the 
individual beams. This is done by using a priori knowledge of the coordinates of the centres of the scatter distributions. The details of this algorithm and why it was chosen are given in Section 4.4. Figure 3.14 shows the extracted radial profiles from the data displayed in Figure 3.13. The difference in peak amplitudes is due to the beams being of slightly different size and intensity.

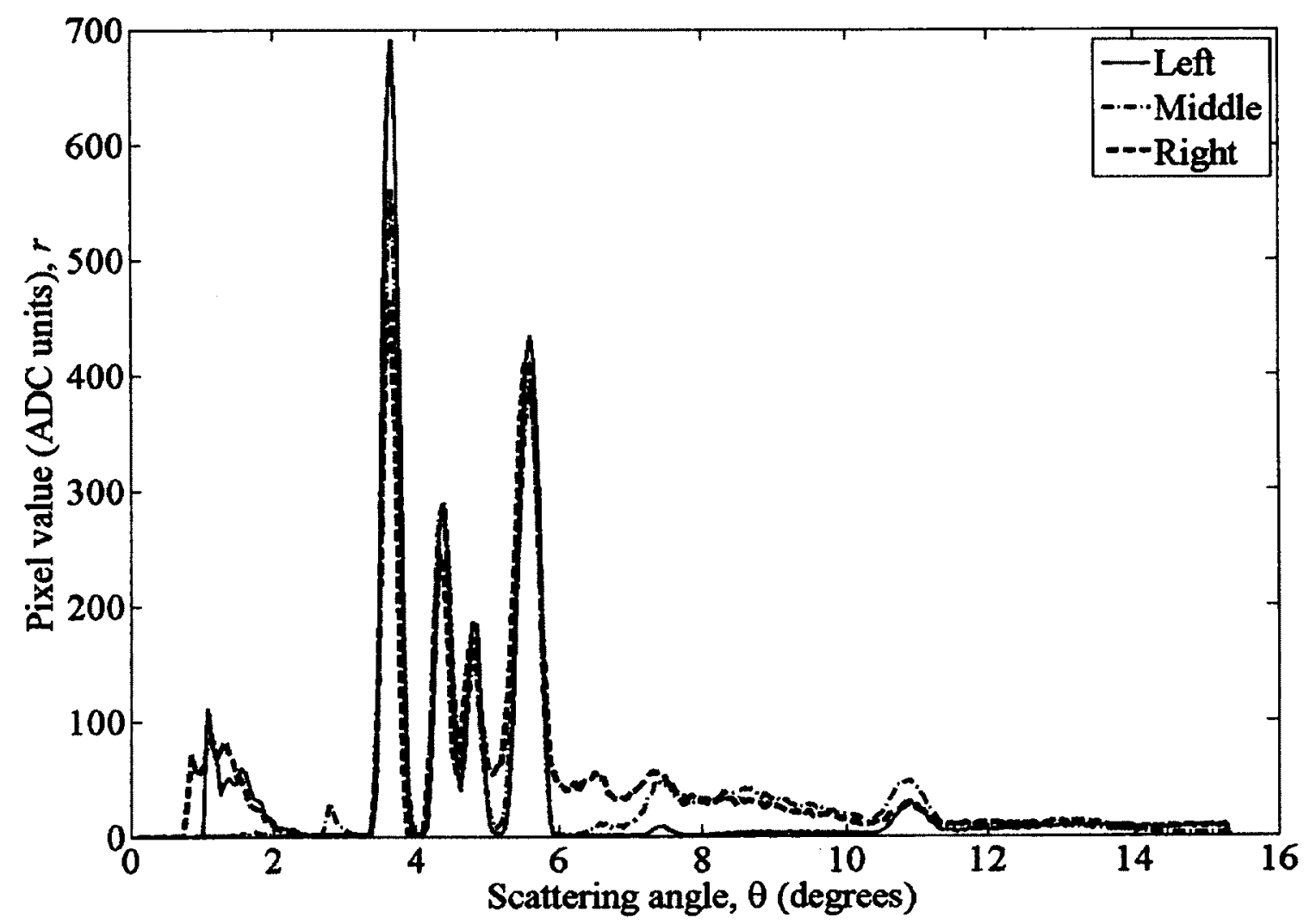

Figure 3.14: The three radial profiles extracted using MLEM from the data shown in Figure 3.13.

Since the phantom used is simply a uniform bar of plastic, the three recovered radial profiles should be identical. As seen in Figure 3.14, however, the peaks from the three different beams are not the same size, which is a result of the three beams being of 
slightly different area (due to the collimator) and/or different intensity (due to the monochromator and non-uniformity of the synchrotron fan beam). To reduce this effect a normalization of the radial profiles, relative to the left beam, was done using the following equation:

$$
r_{i}^{n}=r_{i}\left[\left(\int_{3.25^{\circ}}^{5.95^{\circ}} r_{i} d \theta\right) /\left(\int_{3.25^{\circ}}^{5.95^{\circ}} r_{1} d \theta\right)\right], \quad i=2,3, \ldots
$$

where the superscript $n$ is to represent a normalized radial profile, and the angle range of $3.25^{\circ}$ to $5.95^{\circ}$ was chosen to cover all of the peaks, which will be least affected by background noise. For each configuration of the collimator a new set of normalization coefficients will be needed. Table 3.2 displays the factors by which the radial profiles must be multiplied to normalize them (starting with the left-most first).

Table 3.2: Normalization values due to differences in the beams. Data are listed left to right, where $r_{1}$ is defined to be the left-most beam, resulting in the normalization coefficient being exactly 1 .

\begin{tabular}{|l|l|}
\hline Date of experiment & Multiplicative factors \\
\hline December 2011 & $1.00,1.04,1.08$ \\
\hline May 2012 & $1.00,0.97,1.08,1.08,1.62$ \\
\hline
\end{tabular}

For images from the May 2012 experiment, although the normalization factors were computed, it was found that this normalization was not sufficient (likely due to the large differences in beam amplitude). Therefore the same stitching algorithm described for the primary images was used for the scatter images instead of the normalization factors. 
Re-scaling the data in Figure 3.14 yields the result of Figure 3.15. Although improved over Figure 3.14, the three radial profiles are still not identical. This is either due to imperfections in the radial-profile extraction algorithm, or due to artifacts in the detector's signal. Potential ways to improve the disentangling algorithm are listed as future work in Section 6.2.

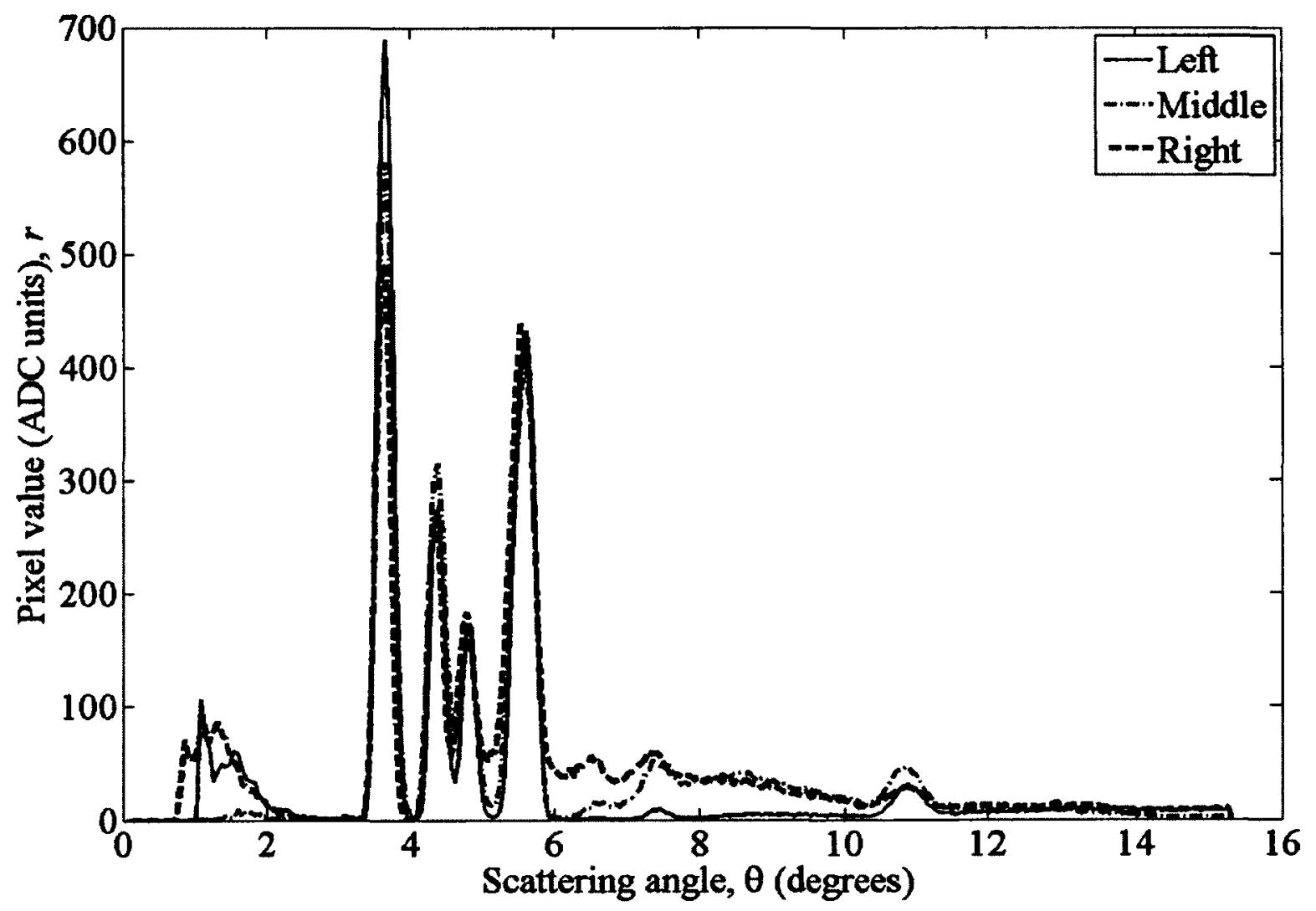

Figure 3.15: Three radial profiles of the uniform plastic bar after normalization.

Once each radial profile has been determined, the unique shape determined by the atomic or molecular structure can be utilized to obtain an image maximizing contrast between two materials. The pixel value for the scatter image is calculated using the following equation: 


$$
p(\mathrm{x}, \mathrm{y})=\int_{\theta_{i}}^{\theta_{f}} r^{n}(\mathrm{x}, \mathrm{y}, \theta) d \theta,
$$

where $\mathrm{x}$ and $\mathrm{y}$ are the horizontal and vertical spatial coordinates, respectively, and $\theta_{i}$ and $\theta_{f}$ are the initial and final angles, respectively. Ideal values for $\theta_{i}$ and $\theta_{f}$ require knowledge of the coherent form factor of the material of interest. With the form factors of two materials the cross sections of these materials can be calculated using Equation 2.2. With these cross sections the angular range which maximizes the contrast between two materials can be computed numerically using Equations 1.4 and 3.2. An important benefit of this imaging technique is that one scan can be used to generate multiple images, simply by varying $\theta_{i}$ and $\theta_{f}$. Generally it is useful for images to be generated which maximize the contrast between some target and background material. Figure 3.16 illustrates how to maximize the contrast between polyethylene and polystyrene.

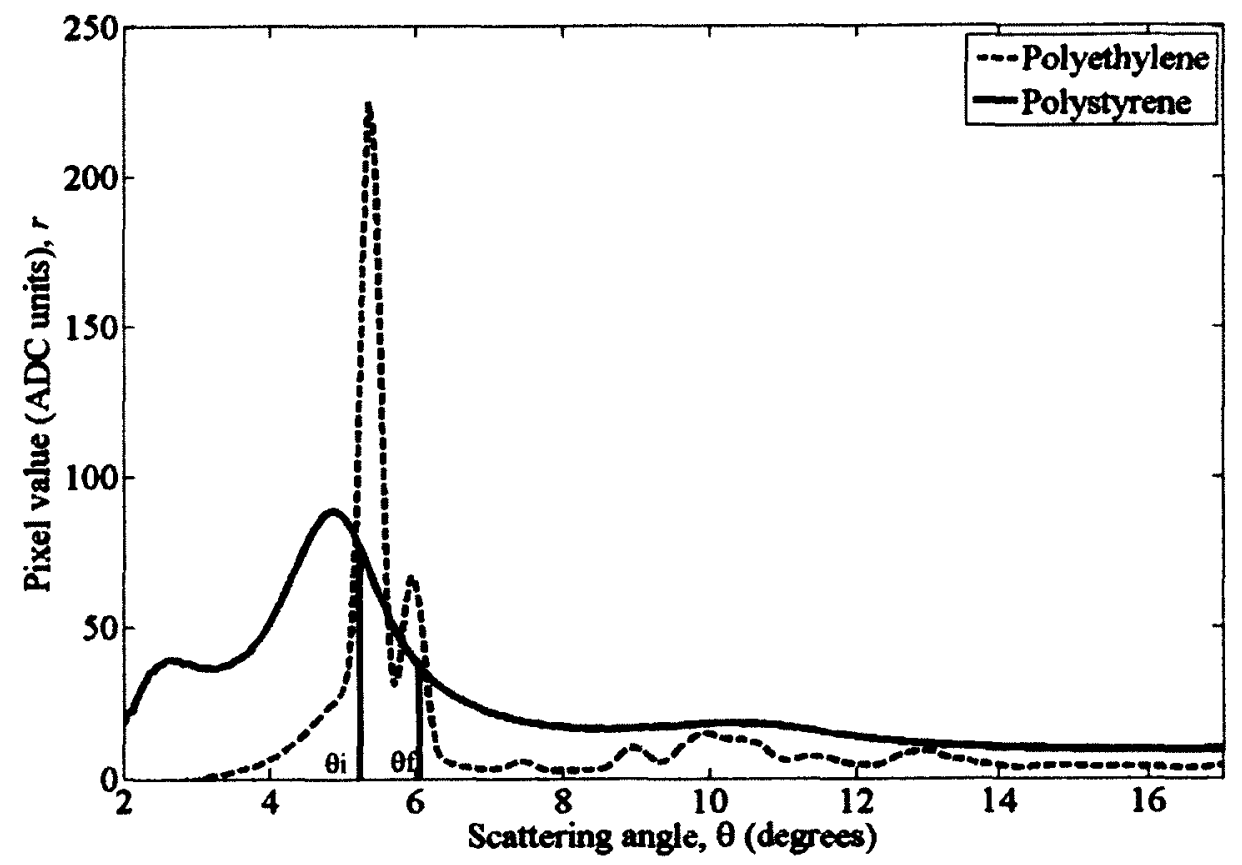

Figure 3.16: Radial profiles of polyethylene and polystyrene. The angular range which would maximize the contrast between these two materials is shown for illustration purposes. 
Ideal angular ranges which maximize the contrast (Equation 1.4) between fat, muscle and bone have been calculated and are displayed in Table 3.3 using the data from King, Landheer and Johns. ${ }^{31}$ The values $1.32^{\circ}$ and $17.12^{\circ}$ are the minimum and maximum measured angles. They vary slightly based on the sample location and tungsten bar placement from one experiment to another.

Table 3.3: Angular ranges to maximize contrast between two biological tissues. Multiple angle ranges are separated by a semicolon.

\begin{tabular}{|l|l|l|}
\hline \multicolumn{1}{|c|}{ Material 1 } & \multicolumn{1}{|c|}{ Material 2 } & \multicolumn{1}{c|}{ Angular range (degrees) } \\
\hline Fat & Muscle & {$[3.50,5.57]$} \\
\hline Fat & Bone & {$[1.328 .52 ; 8.8817 .12]$} \\
\hline Muscle & Bone & {$[1.3217 .12]$} \\
\hline
\end{tabular}

\subsection{Kerma measurement}

The air kerma was measured as a metric to compare to other imaging modalities currently used. From this value the overall effective dose can be calculated for various procedures which can be used as a way to calculate the risk involved in a scan.

Air kerma was measured using $\mathrm{Al}_{2} \mathrm{O}_{3}: \mathrm{C}$ optically-stimulated luminescent dosimeters (OSLDs) cut from Luxel tape (Landauer, Glenwood, Illinois) using a standard hole-punch. The detectors were bleached for 6-8 hours prior to irradiation using a bleaching unit consisting of four $26 \mathrm{~W}$ fluorescent lights in an aluminum box, to ensure vacancy of all electron traps. They were then wrapped individually in light-proof electrical tape. The dosimeter crystals were $7.3 \mathrm{~mm}$ in diameter and $0.4 \mathrm{~mm}$ in thickness. 
Each of the five beams was measured twice for a total of 10 OSLDs. Two additional OSLDs were kept as controls. After return from the synchrotron, the OSLDs were read out using a custom built apparatus. ${ }^{39}$ The crystals were illuminated using $530 \mathrm{~nm}$ emission peak green LEDs (LXML-PM01-0100 Green Rebel LED, Philips Lumileds Light Company, San Jose, California), and the filter had a $405 \mathrm{~nm}$ transmission peak (5113 Optical filter, Kopp Glass Inc., Pittsburgh, Pennsylvania). A photomultiplier tube (P25PC-02 S/N 270, Sens-Tech limited, Langley, United Kingdom) was used to record the counts. Each OSLD was read out at room temperature for $240 \mathrm{~s}$, with the last $20 \mathrm{~s}$ used as the background.

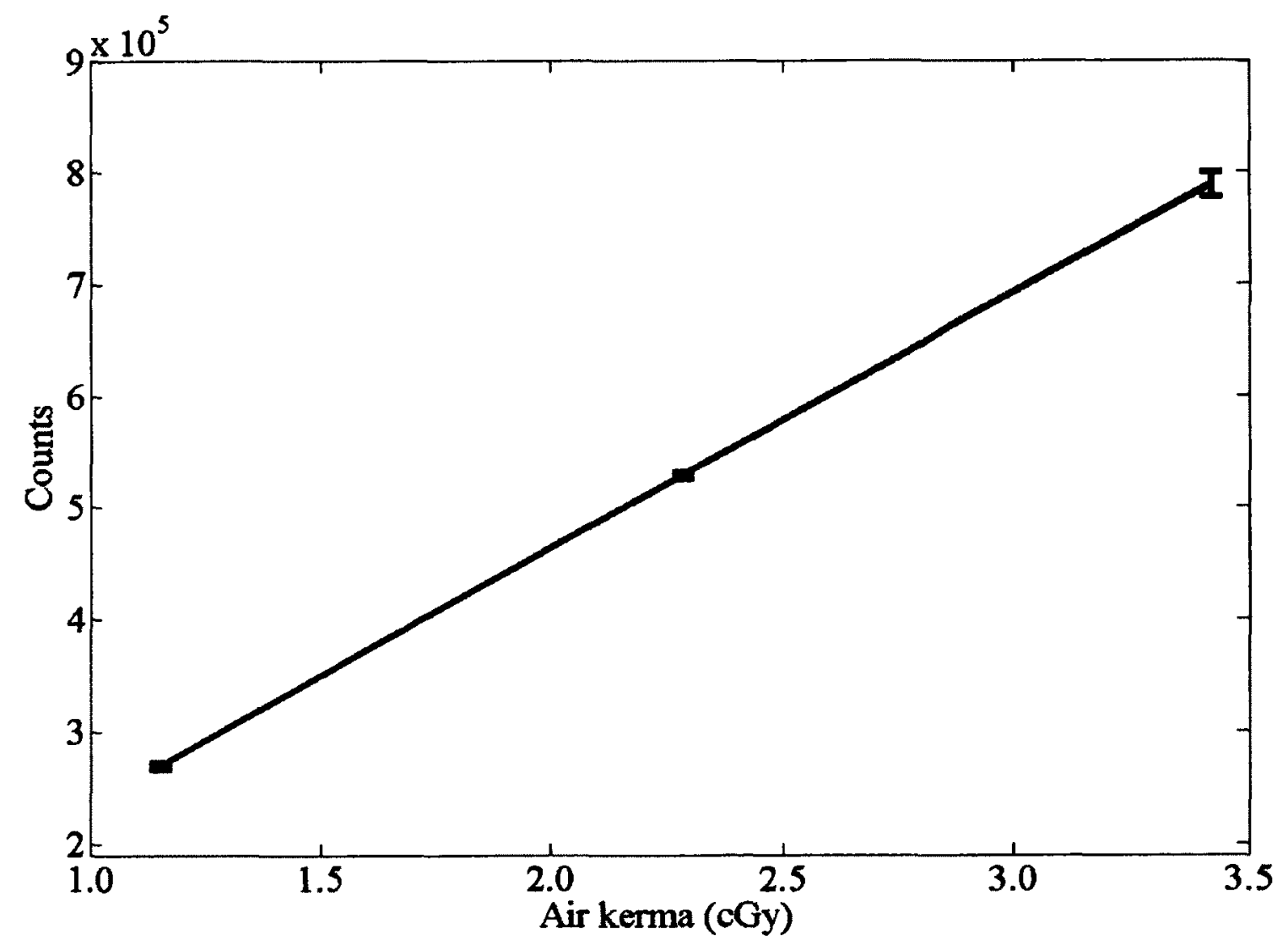

Figure 3.17: Plot of optical read-out photons versus measured air kerma. This is used to calibrate the OSLDs. 
An experiment was performed to calibrate the OSLDs by measuring the air kerma using a calibrated Precision Radiation Measurement (Nashville, Tennesee) Model D-15 $15 \mathrm{~cm}^{3}$ pancake diagnostic air chamber and the number of optical read-out photons from the OSLDs. The source was an x-ray tube at $65 \mathrm{kVp}$. Figure 3.17 is a plot of read-out photons versus air kerma.

At doses below $50 \mathrm{~Gy}$ the response of OSLDs is linear, ${ }^{40}$ so we use a leastsquares regression to fit the data to a linear equation. The number of photons as a function of air kerma is given by:

$$
O=2.3 \times 10^{5} K_{\text {air }}+5.5 \times 10^{3},
$$

where $O$ is the number of optical photon counts and $K_{\text {air }}$ is the air kerma in cGy. 


\section{Chapter 4: Data Processing}

\subsection{Outline}

This chapter describes the algorithms developed to process the data from the flat panel detector. The various filters which were required before any disentangling algorithm could be run are described. This filtering was necessary due to detector damage (defect pixels being unphysically high) or unwanted scatter (i.e., scatter from other objects in the room instead of the desired scatter from the target). The two disentangling algorithms necessary for multi-beam experiments (least-squares and MLEM) are developed and tested.

\subsection{Filtering}

For the data from the December 2011 experiment significant filtering had to be done due to artifacts appearing on the detector from unwanted scatter. For these images the raw data from the detector are first median filtered to remove any defect pixels. We designate the result as the matrix $\boldsymbol{D}$. A uniform plastic bar was used to evaluate the disentangling algorithms and to find the centre locations of the three beams; a photograph of it is given in Figure 3.12. The median filtered scatter pattern of this plastic bar using three pencil beams is shown in Figure 4.1.

The data from December 2011 are then high-pass filtered to remove the lowfrequency artifact on the detector. In Figure 4.1 the bottom half of the scatter pattern is significantly darker than the top half due to this low-frequency artifact. This step was not 
necessary for the May 2012 experiment since we used additional shielding to remove the unwanted scatter. The filtered scatter pattern is given by the following equation:

$$
\boldsymbol{T}=\boldsymbol{D}-\boldsymbol{D} *\left[\frac{a}{2 \pi \sigma^{2}} \exp \left[\frac{-\left(\mathrm{x}^{2}+\mathrm{y}^{2}\right)}{2 \sigma^{2}}\right] \Pi\left(\frac{\mathrm{x}}{l}\right) \Pi\left(\frac{\mathrm{y}}{l}\right)\right],
$$

where $\sigma$ is the standard deviation of the Gaussian, $l$ is the width of the rectangle function $\Pi$, and $a$ is a constant to ensure the kernel is properly normalized. The kernel is found by iterative manual adjustment to remove as much of the artifacts as possible without affecting the signal. The convolution kernel ( $\sigma$ and $l$ ) can be adjusted depending on the amount of low-frequency information in the system. Then each column of the filtered scatter pattern, $T$, has the mean of the top 20 rows subtracted from it to remove the bandlike structure present from the physical structure of the detector. The final result of the filtering is designated by $\boldsymbol{S}$ :

$$
S_{i, j}=T_{i, j}-\frac{1}{20} \sum_{i^{\prime}=1}^{20} T_{i^{\prime}, j} .
$$

Figure 3.13 is the median filtered, high-pass, and background subtracted scatter pattern for a three-beam exposure of the plastic bar shown in Figure 3.12. For all other experiments a simple median filter was sufficient.

\subsection{Least-squares}

For reference the filtered scatter pattern of a single beam exposure of the same plastic bar is given in Figure 4.2 (from the May 2012 experiment), with its extracted radial profile given in Figure 4.3. This radial profile is used as the gold standard as it does not require any disentangling algorithm. 


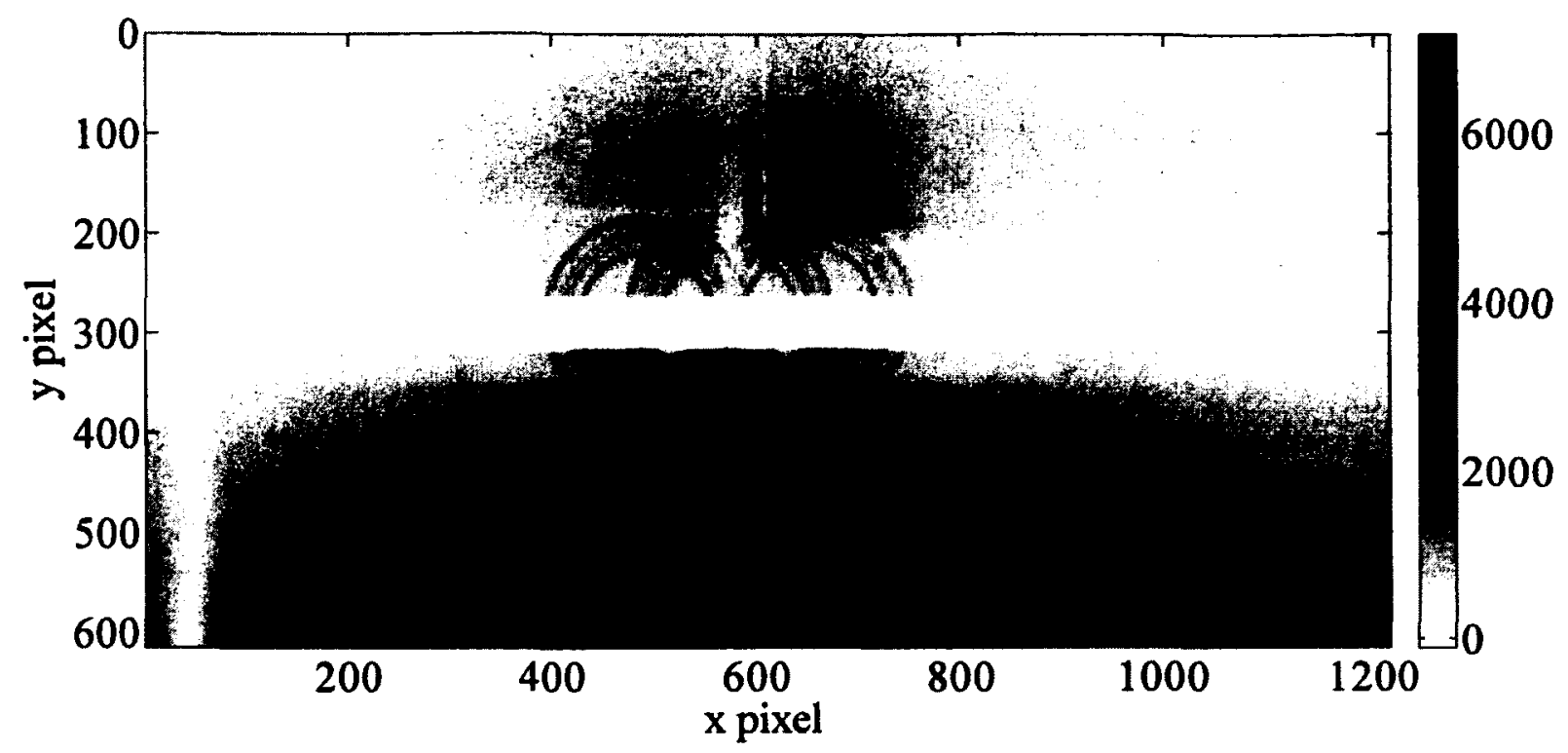

Figure 4.1: Median-filtered scatter pattern of a plastic bar. Three individual pencil beams were used. These data correspond to $D$ in Equation 4.1.

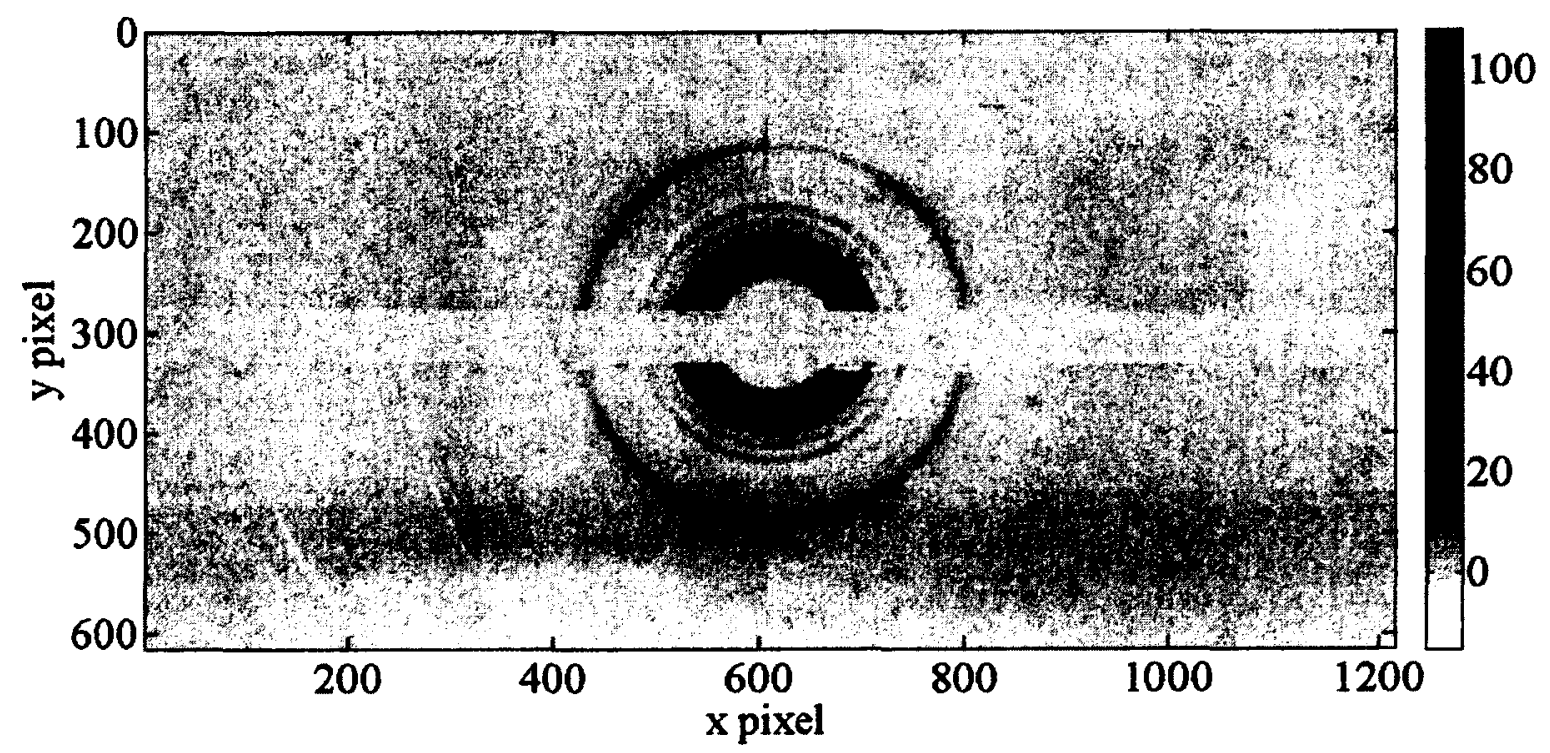

Figure 4.2: Median filtered data of a single beam exposure of a uniform plastic bar. Some damaged columns are noticeable but are excluded in the algorithm. 


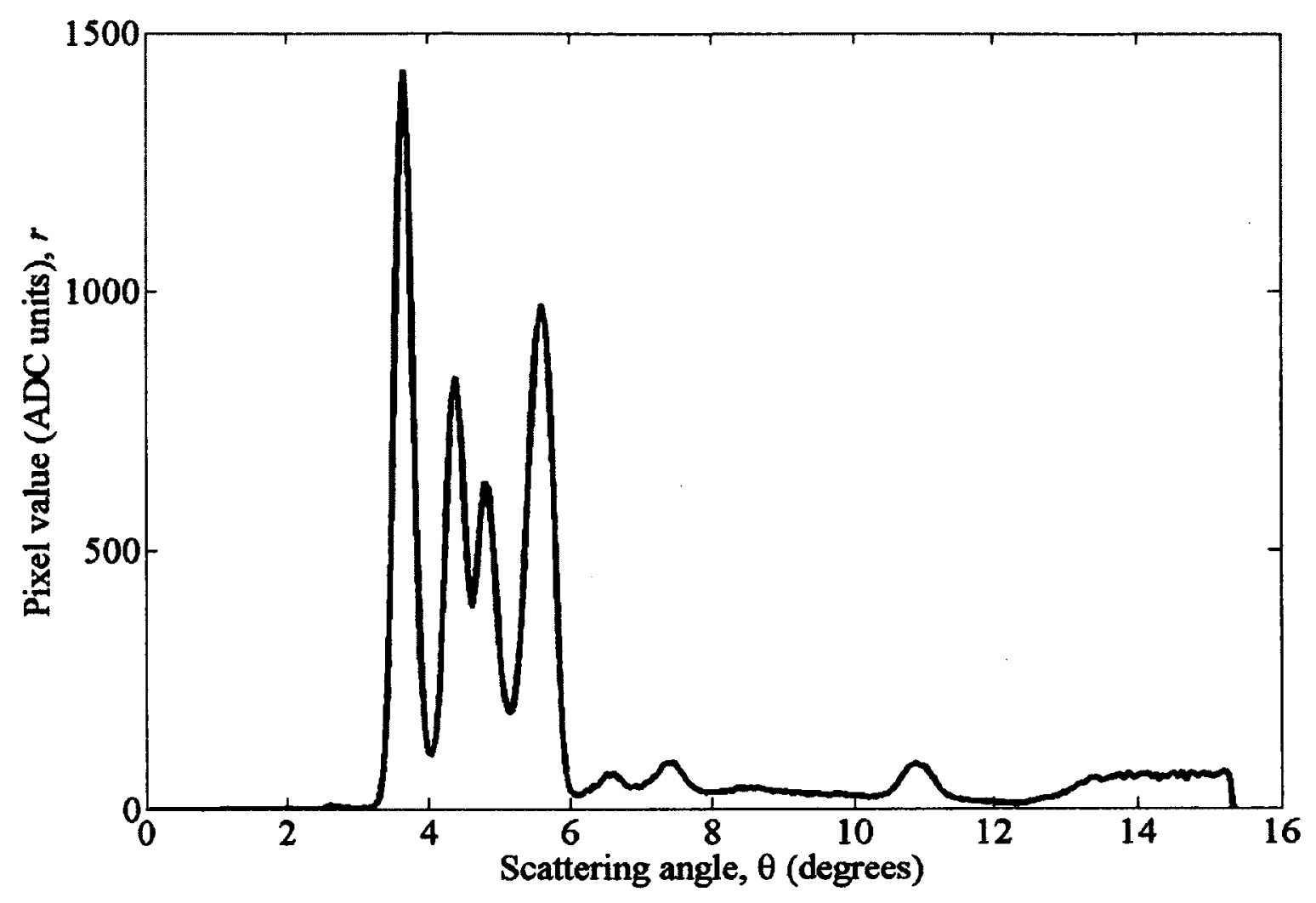

Figure 4.3: Radial profile of the single beam scatter pattern Figure 4.2 from the uniform plastic bar. This radial profile is used as gold standard for the disentangling algorithms.

A least-squares method was developed to extract the individual superimposed scatter patterns shown in Figure 3.13. Let $S$ represent the filtered data from the flat panel digital detector. If $S$ is of size $n$ by $m$ then we let the vector $b$ be the column vector of $S$. The column vector, $b$, is given by:

$$
b=\left(\begin{array}{c}
S_{1,1} \\
S_{2,1} \\
S_{3,1} \\
\vdots \\
S_{n, 1} \\
S_{1,2} \\
S_{2,2} \\
\vdots \\
S_{n, m}
\end{array}\right)
$$


$\boldsymbol{A}$ is defined to be the system matrix (scattering operator) which maps our radial profile row vector, $r$, to the measured data $b$ :

$$
A r=b .
$$

$\boldsymbol{A}$ is determined by the geometry of the setup. In particular a priori knowledge of the centres of the beam must be known. These are found by obtaining a single-beam scatter pattern of the plastic bar and running an algorithm which varies the centre location and finds the position where the peaks are sharpest. This position is the centre of the scatter pattern. This must be done individually for each beam.

If we are looking for the least-squares solution to this equation, $r$, it is given by rearranging Equation 4.4 to give:

$$
r=\left(A^{t} A\right)^{-1}\left(A^{t} b\right)
$$

Using Equation 4.5 to obtain the least-squares solution for $r$ from the data displayed in Figure 3.13 the three radial profiles shown in Figure 4.4 are obtained. 


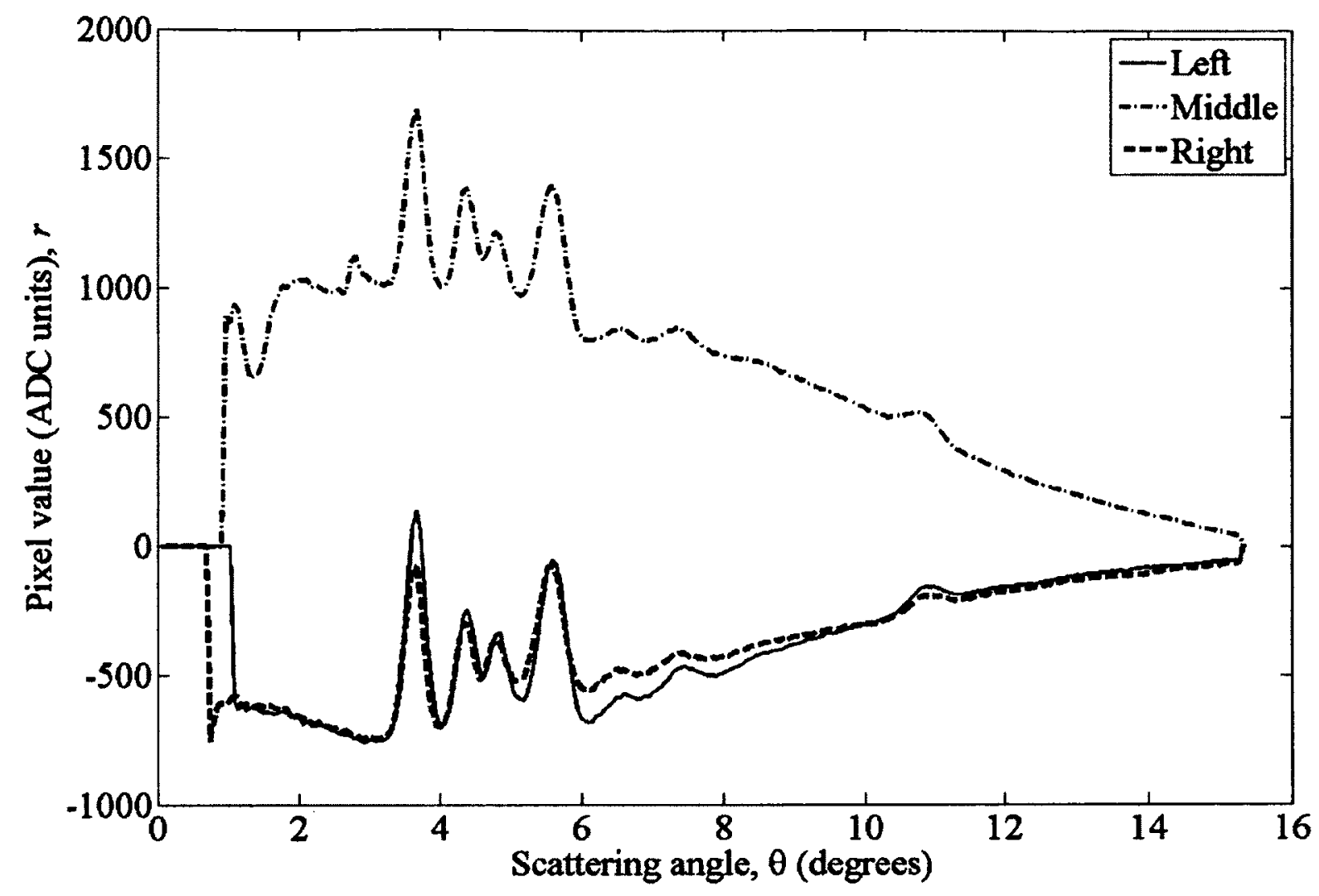

Figure 4.4: Three radial profiles extracted using least-squares from the data displayed in Figure 3.13 .

Although these radial profiles give the least-squares solution to the problem they are not physically realistic (there should be no negative values). It is evident when comparing Figure 4.4 to Figure 4.3 that the large negative regions are not a realistic solution and so we investigated the non-negative least-squares (NNLS) solution using the built-in MATLAB function Isqnonneg to Equation 4.4. Using this technique the result of Figure 4.5 is obtained. 


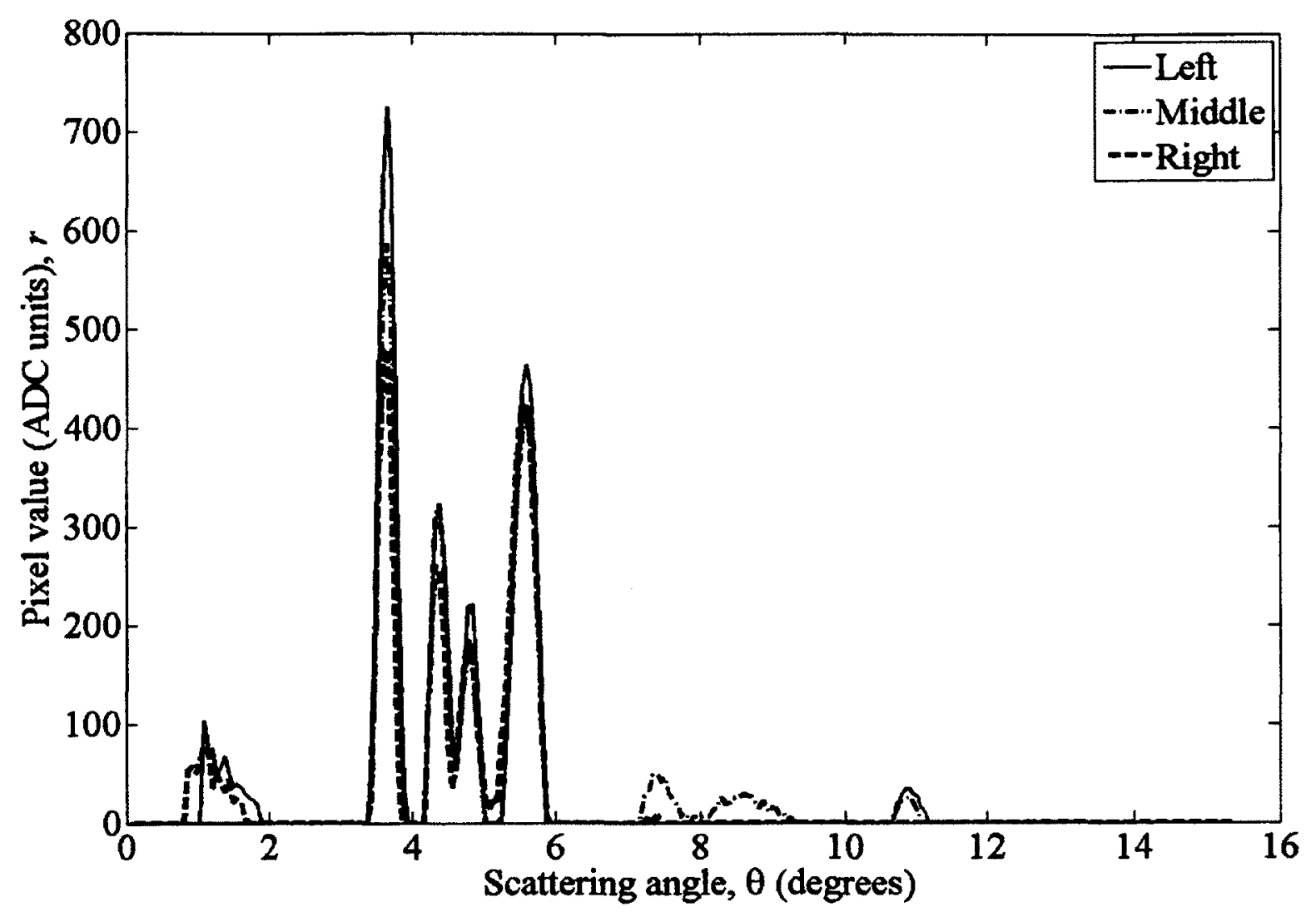

Figure 4.5: Three radial profiles extracted from the data shown in Figure 3.13 using a non-negative least-squares algorithm.

This solution is much closer to the desired result but it still has significantly different features when compared with the single beam radial profile (Figure 4.3). Additionally there is no physical reason why the NNLS solution to Equation 4.4 is the desired one. For these reasons we developed an alternative maximum-likelihood expectation-maximization algorithm.

\subsection{Maximum-Likelihood Expectation Maximization (MLEM)}

An alternative solution to Equation 4.4 is the maximum-likelihood expectation maximization solution. This was initially developed for emission and transmission 
tomography by Lange and Carson, ${ }^{41}$ but can be applied here as a disentangling algorithm to extract the independent overlapping radial profiles. We can alternatively express Equation 4.4 as:

$$
\bar{b}_{i}=\sum_{j} A_{i, j} r_{j}
$$

where $A_{i, j}$ is the $i^{\text {th }}$ row, $j^{\text {th }}$ column element of the system matrix $A, r_{j}$ is the $j^{\text {th }}$ element in the radial profile vector $r$, and $\bar{b}_{i}$ is the expected number of counts in the $i^{\text {th }}$ element of the column vector $b$. Assuming the number of counts in a bin is a Poisson random variable, the probability that there are $b_{i}$ counts in the $i^{\text {th }}$ position of the vector $b$ is given by:

$$
P\left(b_{i}\right)=\frac{e^{-\bar{b}_{i}} \bar{b}_{i}^{b_{i}}}{b_{i} !}
$$

Since the Poisson variables are independent the overall probability, or likelihood function, is the product of all the individual probability elements:

$$
L(r)=\prod_{i} \frac{e^{-\bar{b}_{i} \bar{b}_{i} b_{i}}}{b_{i} !} .
$$

Taking the logarithm and substituting in Equation 4.6 we obtain:

$$
\ln [L(r)]=\sum_{i}\left[-\sum_{j} A_{i, j} r_{j}+b_{i} \ln \sum_{j} A_{i, j} r_{j}-\ln \left(b_{i} !\right)\right] .
$$

The MLEM solution for $r$ can be solved by taking the derivative of the log-likelihood function, with respect to $r_{j}$ and setting that equal to zero (which maximizes likelihood, since the logarithm is a monotonic function): 


$$
\frac{\partial \ln [L(r)]}{\partial r_{j}}=-\sum_{i} A_{i, j}+\sum_{i} \frac{b_{i}}{\sum_{t} A_{i, t} r_{t}} A_{i, j}=0 .
$$

Due to the difficulty of solving Equation 4.10 analytically for $r$ we look for an iterative solution. Rearranging this and multiplying both sides by $r_{j}$ the following equation is obtained:

$$
r_{j}=\frac{r_{j}}{\sum_{i} A_{i, j}}\left(\sum_{i} \frac{b_{i}}{\sum_{t} A_{i, t} r_{t}} A_{i, j}\right) .
$$

This equation is used to create an iterative MLEM algorithm, which converges on the most-likely solution to Equation 4.4 :

$$
r_{j}^{(k+1)}=\frac{r_{j}^{k}}{\sum_{i} A_{i, j}}\left(\sum_{i} \frac{b_{i}}{\sum_{t} A_{i, t} r_{t}^{k}} A_{i, j}\right),
$$

where the superscript $k$ is used to denote the iteration number. The factor $\left(\sum_{i} \frac{b_{i}}{\sum_{t} A_{i, t} r_{t}^{k}} A_{i, j}\right)$ in Equation 4.12 is the backprojection of the ratio of the real data, $b$, to the forward projection of the previous iteration's radial profile. The factor $\sum_{i} A_{i j}$ in the denominator is simply for normalization. Equation 4.12 is equivalent to the more intuitive method explained in the following paragraph. The matrix method, however, has a computational advantage if the system matrix $\boldsymbol{A}$ has already been characterized. For the first iteration the solution, $r$, is defined to be a vector of all ones.

The MLEM solution of Equation 4.4 can alternatively be done iteratively via four operations: 1. A scatter pattern is generated from the radial profile (this is initially all ones). 2. The filtered data, $S$, are divided by the generated scatter pattern, element by element, resulting in an update scatter ratio. 3 . The update scatter pattern is averaged over concentric circles resulting in multiplicative radial profile factors. 4. The previous 
iteration's radial profiles are multiplied by the multiplicative radial profile factors, resulting in the new radial profile. The whole procedure iterates until the rms value of the radial profiles changes by less than $10 \%$. The stopping criterion used is the rms change from one iteration to the previous, defined as:

$$
\mathrm{rms}=\sqrt{\frac{1}{n m} \sum_{j=1}^{m} \sum_{i=1}^{n}\left(\frac{r_{i, j}^{k+1}}{r_{i, j}^{k}}-1\right)^{2}},
$$

where $r$ is the radial profile, $j$ is a position index on the detector, $i$ is the index of beam number and the superscript $k$ is used to denote the iteration number. A pictorial representation of the disentangling algorithm is shown in Figure 4.6.

Applying the algorithm explained in Figure 4.6 to the data shown in Figure 3.13 the three radial profiles shown in Figure 4.7 are obtained. It should be noted that the profiles displayed are all of the same plastic but all 3 beams are not the exact same size or intensity, resulting in a difference in amplitude. A normalization was done to correct this effect. Changing the convolution kernel can provide a better result for sharp peaks such as these but will filter out low-frequency information. A forward projection of the data displayed in Figure 4.7 is shown in Figure 4.8.

Figure 4.9 is the five-beam exposure of the same plastic bar, and Figure 4.10 shows the five normalized radial profiles extracted from the data, with the position counting starting from the left. Figure 4.11 is a plot of the rms change versus iterations completed, for the disentanglement of the data shown in Figure 4.9. This algorithm typically takes between 5 and 10 iterations to reach the finishing criterion, depending on how clean the scatter pattern is. For Figure 4.11 the loop would terminate after 6 iterations. The upper bound is set at 25 iterations to avoid a computational snag. 

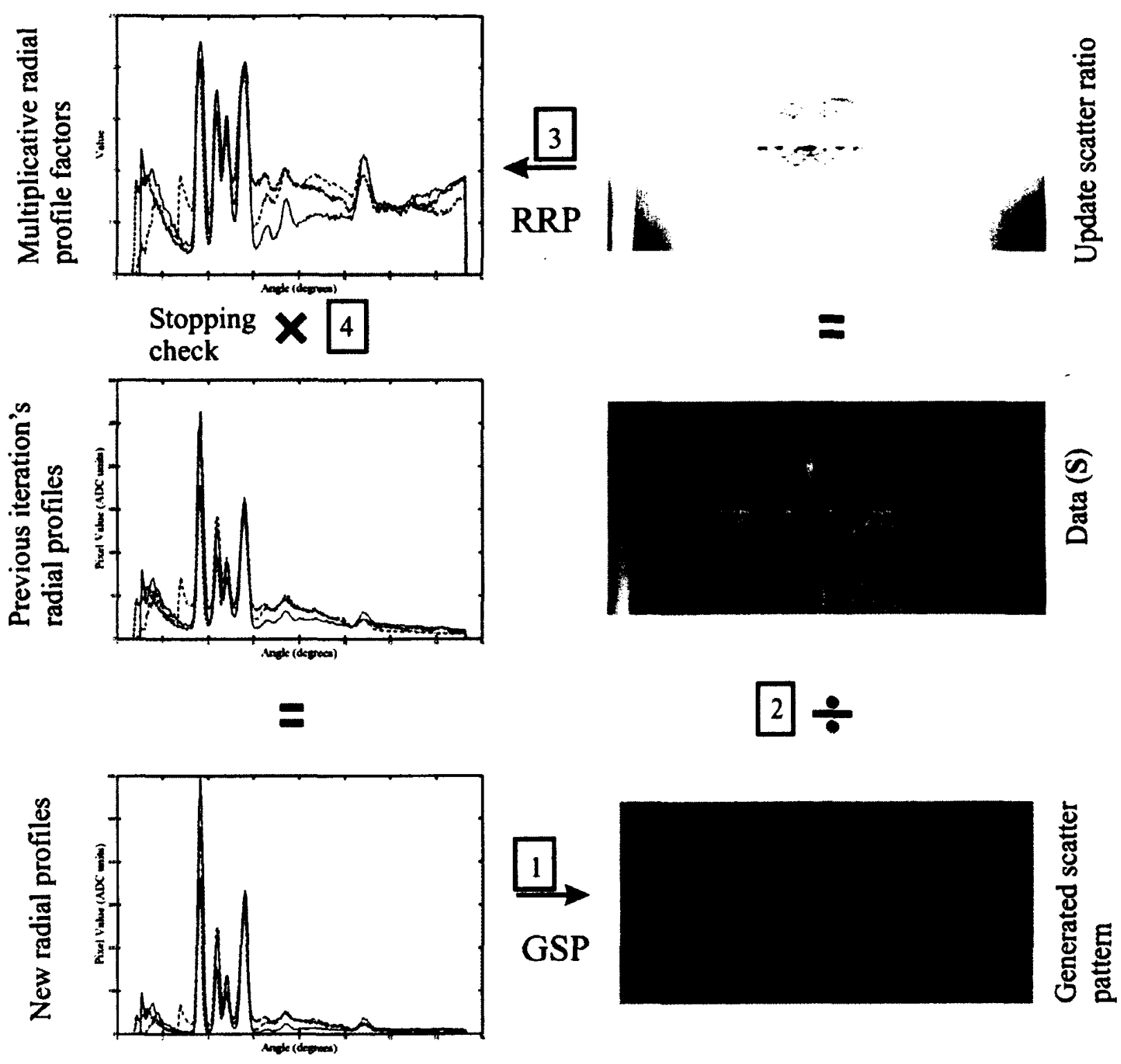

Figure 4.6: Flow chart representation of MLEM algorithm used to obtain multiple radial profiles from overlapped scatter patterns. The acronyms GSP and RRP stand for Generate Scatter Pattern and Recover Radial Profiles. The first step is to generate the scatter pattern from the radial profiles (initially all ones). The second step is to divide the data by the generated scatter pattern, resulting in the update scatter ratio. The third step is to recover the radial profiles from this update scatter ratio by averaging over concentric circles. These are the multiplicative radial profile factors. The fourth step is to multiply the previous iteration's radial profiles by the multiplicative radial profile factors. This results in the new radial profiles and the cycle starts again. At step 4 the stopping criterion is evaluated and if the rms change of the radial profile from one iteration to the next is less than $10 \%$ the looping terminates. 


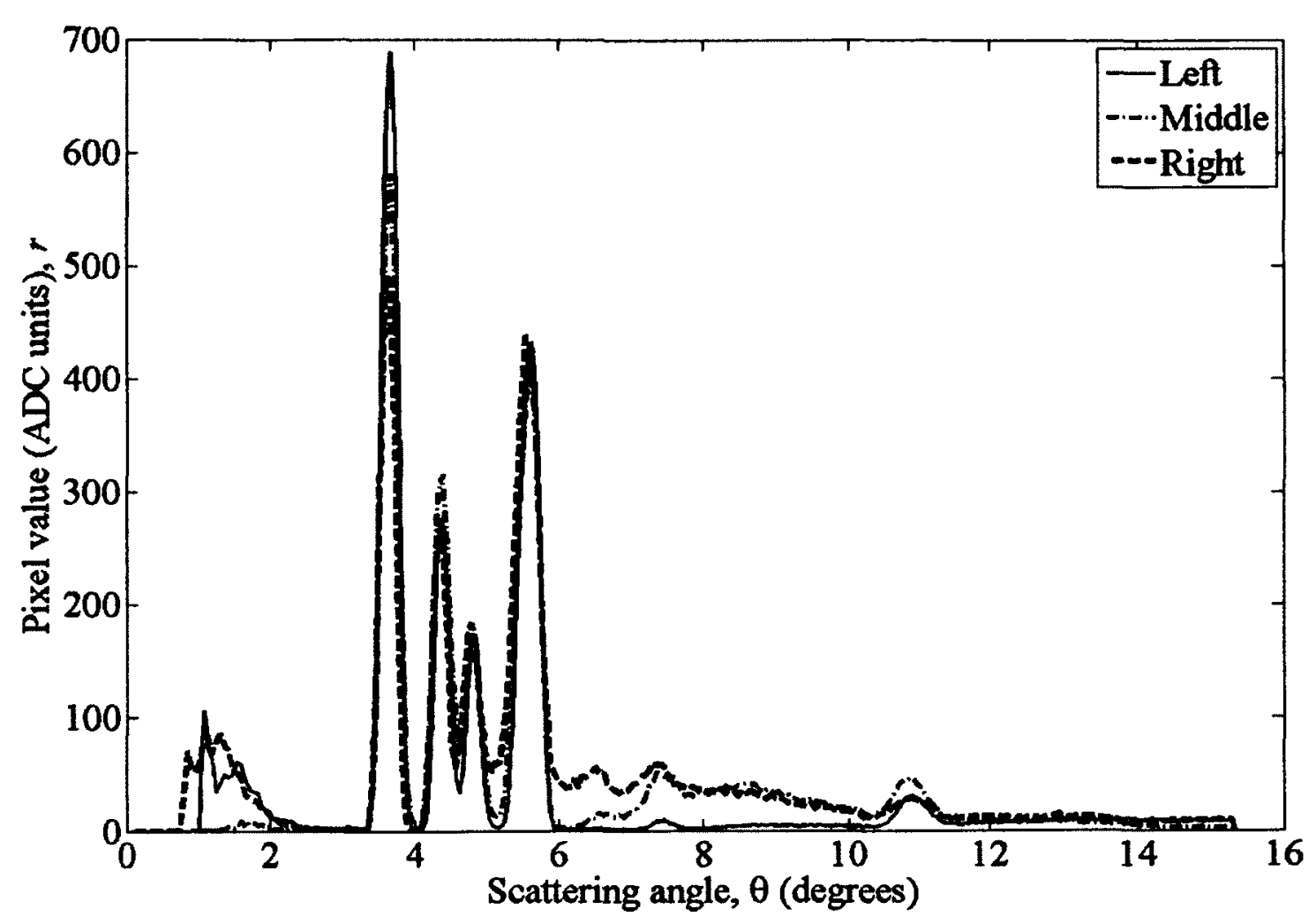

Figure 4.7: The three normalized radial profiles extracted using MLEM from the data shown in Figure 3.13.

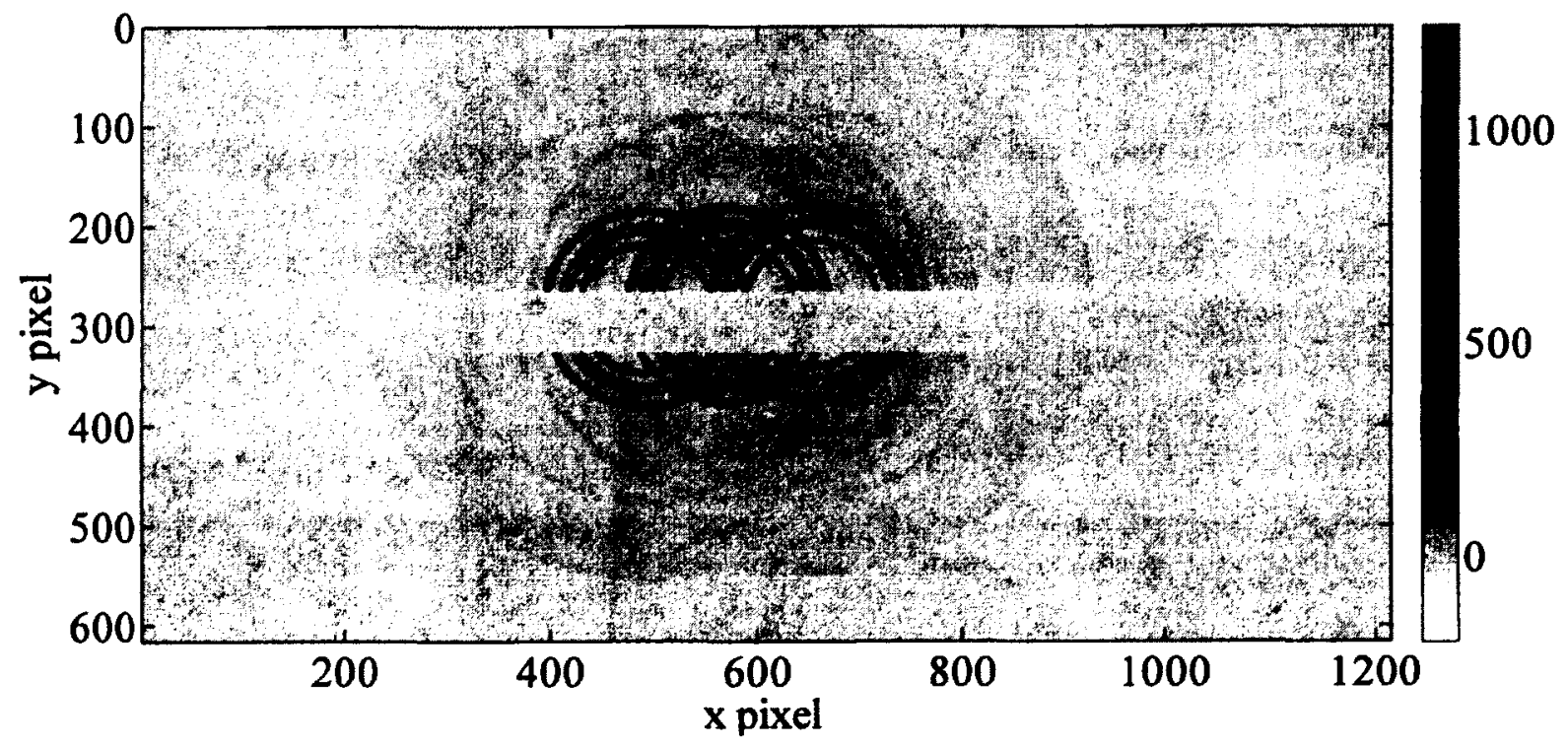

Figure 4.8: Forward projection of the three radial profiles shown in Figure 4.7. This should be similar to the raw data displayed in Figure 3.13. 


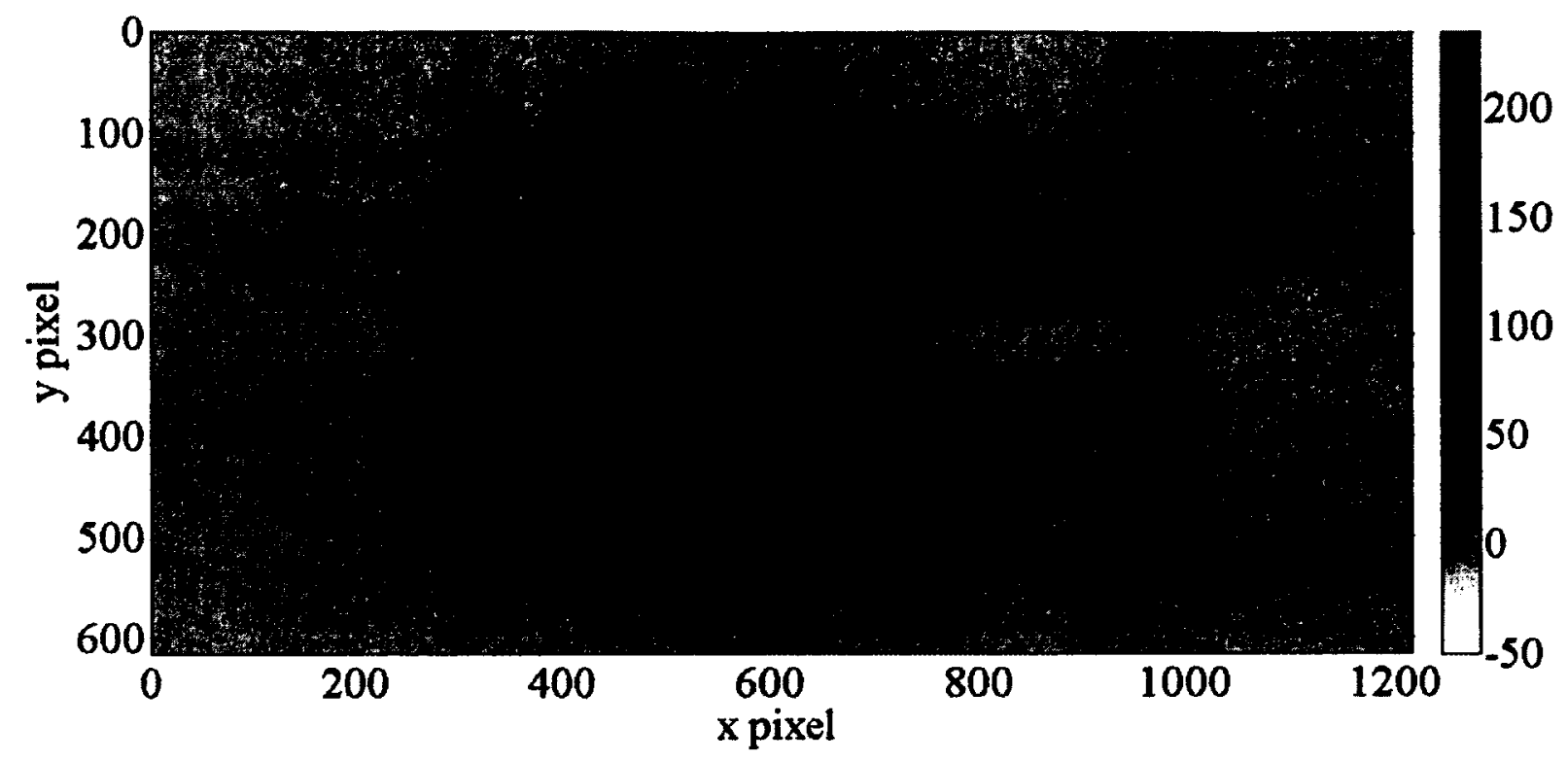

Figure 4.9: Median-filtered five-beam exposure of uniform plastic bar.

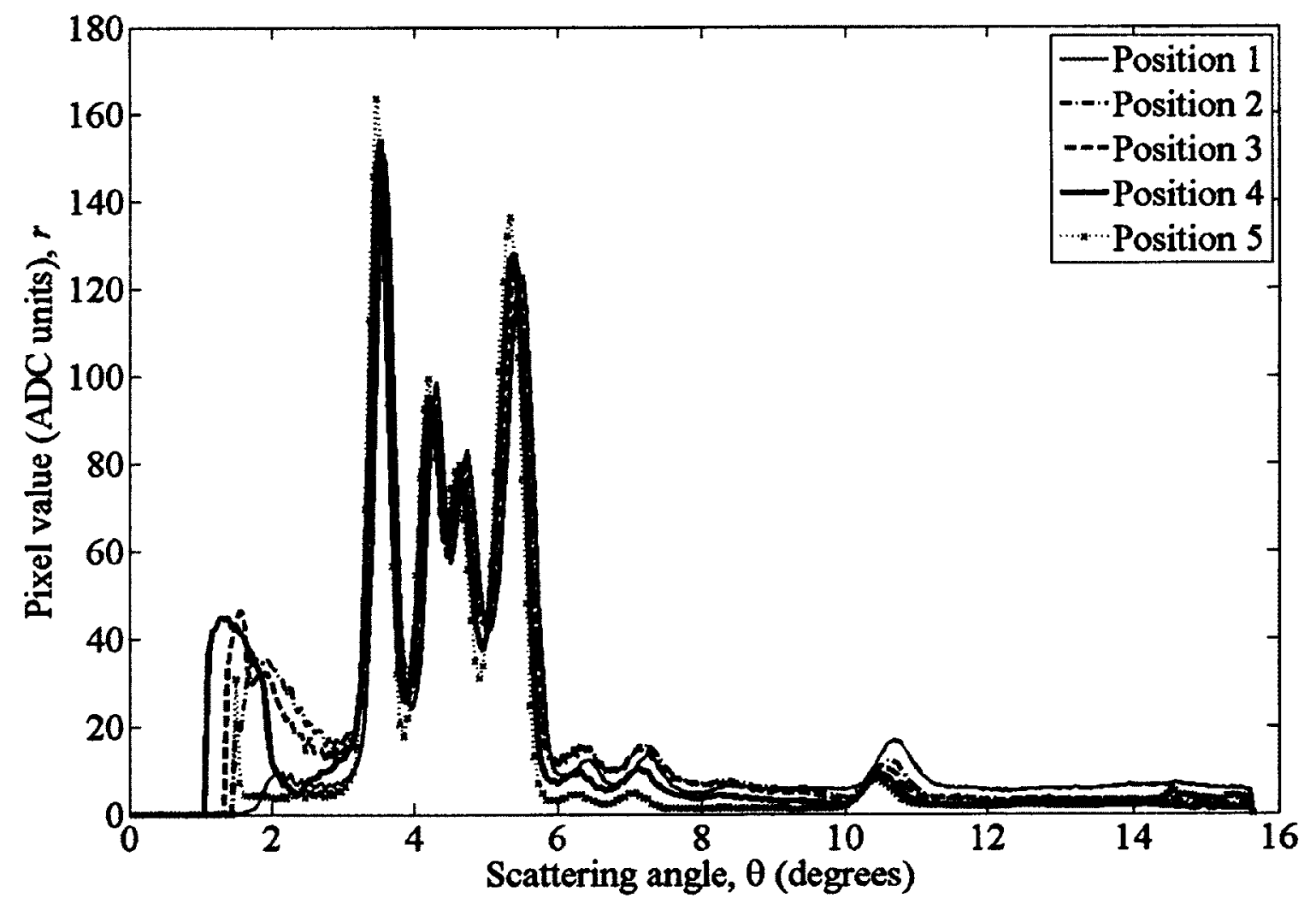

Figure 4.10: Five normalized radial profiles extracted from the data displayed in Figure 4.9. The position counting starts from the left. 


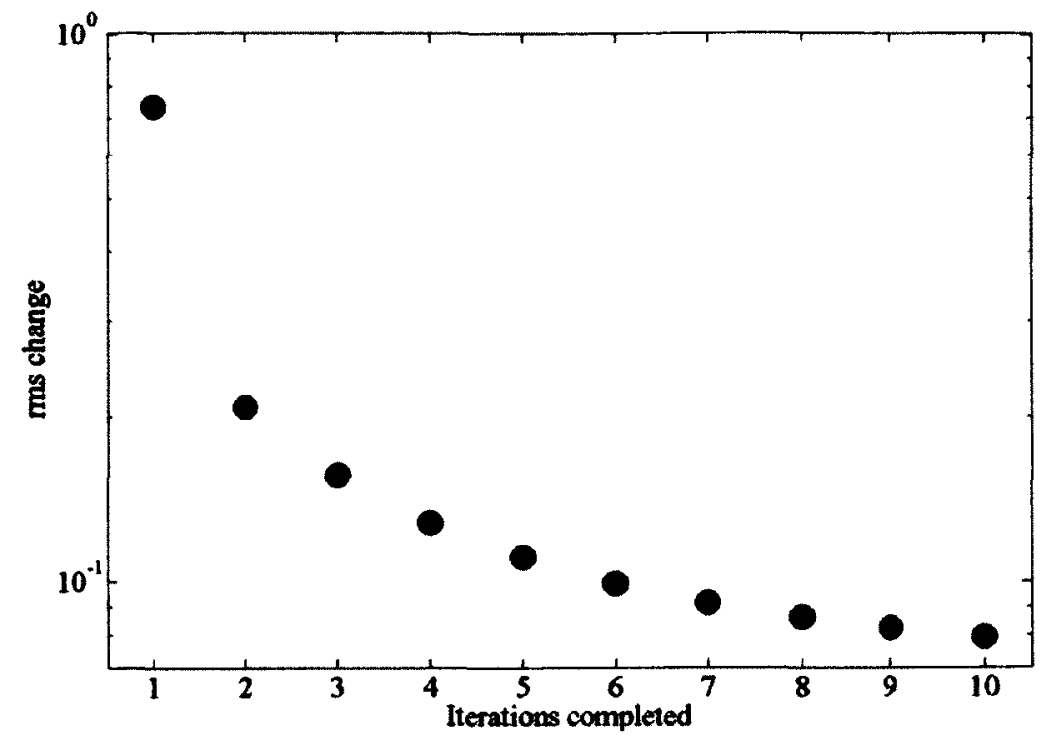

Figure 4.11: Semi-log plot of rms change from one iteration to the next versus iterations completed. 


\section{Chapter 5: Results}

\subsection{Outline}

The results are given in the chronological order they were obtained in. This is also the logical progression as we successively increased the number of beams. Table 5.1 gives a brief outline of the type of phantom, the date of the experiment, total scan duration, pixel beam-time, size of the image and scan time per pixel. The pixel beam-time is chosen in increments of $33 \mathrm{~ms}$ (one detector readout frame) to provide reasonable statistics while minimizing the scan time. Throughout this chapter both scatter and primary images for single and multiple beams are shown of tissue phantoms and plastic/tissue phantoms. The image quality and the scan time per pixel improved with each subsequent visit. All contrasts (the metric used to compare image quality) in this thesis are calculated by averaging a pixel region deemed (by inspection) to be of a particular tissue, and using Equation 1.4.

\subsection{Single beam}

Single beam results are the gold standard for this technique as they do not require any disentangling algorithm. All that is required to extract the radial profile is averaging over concentric circles (which the MLEM algorithm described in Section 4.4 reduces to in the case of one beam). For biological samples the angular range which maximizes fat to muscle is generally used as coherent scatter imaging has much greater inherent soft-tissue contrast, whereas primary is much better for contrast between bone and soft tissues. 
Figure 5.1 shows a porcine phantom which we used to make both primary and scatter images. Figure 5.2, Figure 5.3 and Figure 5.4 are the median-filtered scatter patterns for fat, muscle and bone regions of this phantom, respectively, with a beam time of $3.3 \mathrm{~s}$. There are clearly significant differences between all three tissues; in particular there is a sharp low-angle peak for fat, a very sharp higher angle peak for bone and a smoother pattern for muscle. The radial profiles of these three scatter patterns are shown in Figure 5.5.

Table 5.1: Summary of several characteristics of images presented in this chapter.

\begin{tabular}{|l|l|l|l|l|l|l|}
\hline $\begin{array}{l}\text { Figure } \\
\text { numbers }\end{array}$ & $\begin{array}{l}\text { Date of } \\
\text { experiment }\end{array}$ & $\begin{array}{l}\text { Image } \\
\text { description }\end{array}$ & $\begin{array}{l}\text { Total } \\
\text { scan } \\
\text { duration } \\
\text { (h) }\end{array}$ & $\begin{array}{l}\text { Pixel } \\
\text { beam } \\
\text {-time } \\
\text { (ms) }\end{array}$ & $\begin{array}{l}\text { Image } \\
\text { array (rows } \\
\text { by } \\
\text { columns) }\end{array}$ & $\begin{array}{l}\text { Scan } \\
\text { time } \\
\text { per } \\
\text { pixel } \\
\text { (s) }\end{array}$ \\
\hline $5.6-5.8$ & June 2011 & $\begin{array}{l}\text { 1-beam image } \\
\text { of porcine } \\
\text { tissue phantom }\end{array}$ & 10 & 3300 & $58 \times 50$ & 12 \\
\hline $5.10-5.13$ & $\begin{array}{l}\text { December } \\
2011\end{array}$ & $\begin{array}{l}\text { 2-beam image } \\
\text { of tissue/plastic } \\
\text { phantom }\end{array}$ & 4 & 3300 & $50 \times 42$ & 6 \\
\hline $5.15-5.16$ & $\begin{array}{l}\text { December } \\
2011\end{array}$ & $\begin{array}{l}\text { 1-beam image } \\
\text { of porcine } \\
\text { tissue phantom }\end{array}$ & 7 & 3300 & $50 \times 42$ & 12 \\
\hline $5.17-5.18$ & $\begin{array}{l}\text { December } \\
2011\end{array}$ & $\begin{array}{l}\text { 2-beam image } \\
\text { of porcine } \\
\text { tissue phantom }\end{array}$ & 6 & 3300 & $50 \times 42$ & 6 \\
\hline 5.20 & May 2012 & $\begin{array}{l}\text { 1-beam image } \\
\text { of porcine } \\
\text { tissue phantom }\end{array}$ & 4 & 990 & $58 \times 50$ & 5 \\
\hline 5.21 & May 2012 & $\begin{array}{l}\text { 3-beam image } \\
\text { of porcine } \\
\text { tissue phantom }\end{array}$ & 2 & 990 & $58 \times 60$ & 2 \\
\hline $5.22-5.25$ & May 2012 & $\begin{array}{l}\text { 5-beam image } \\
\text { of porcine } \\
\text { tissue phantom }\end{array}$ & 1 & 990 & $58 \times 50$ & 1 \\
\hline
\end{tabular}




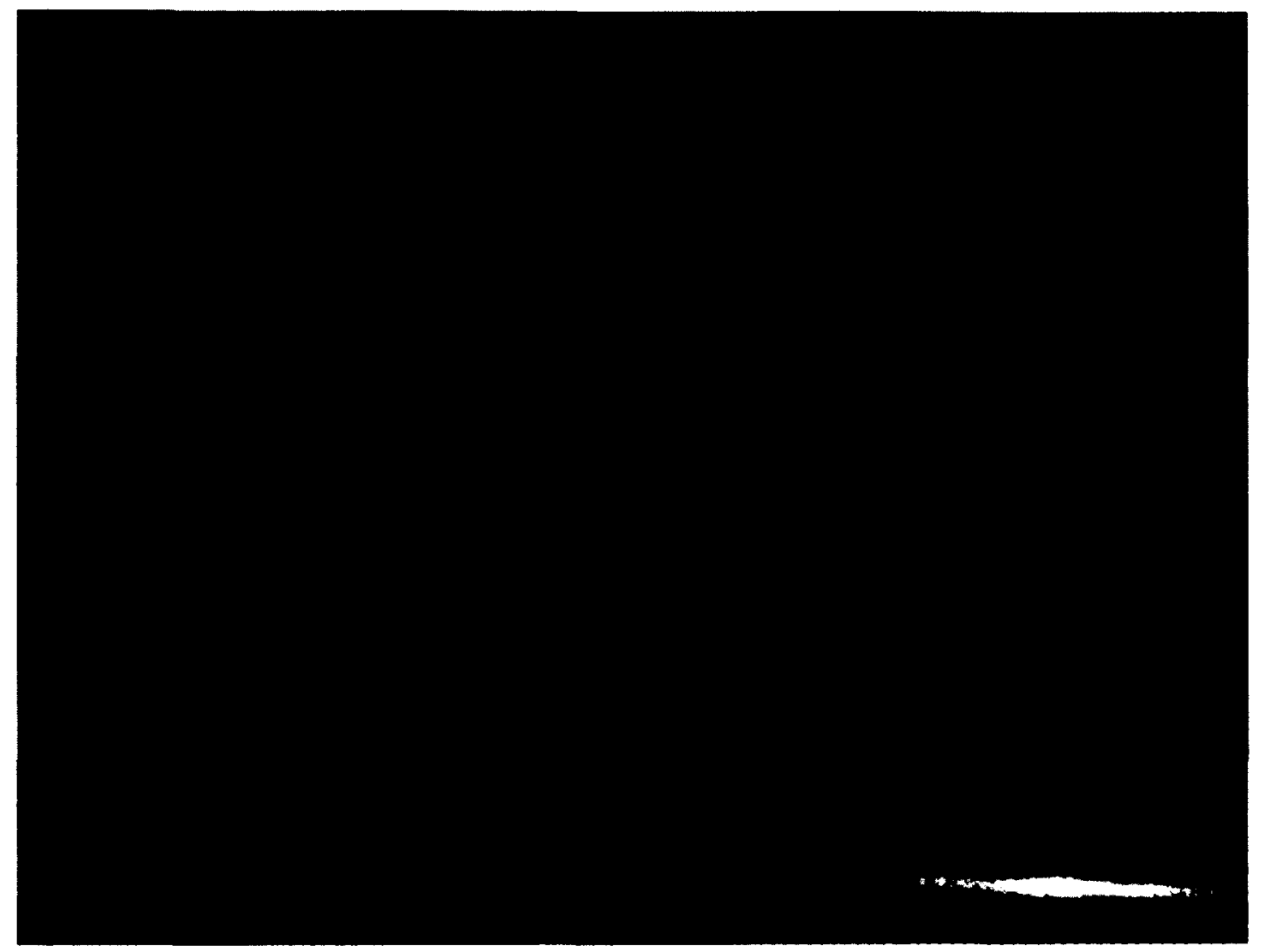

Figure 5.1: Photograph of porcine phantom. Figures 5.2 - 5.8 were made by imaging this sample.

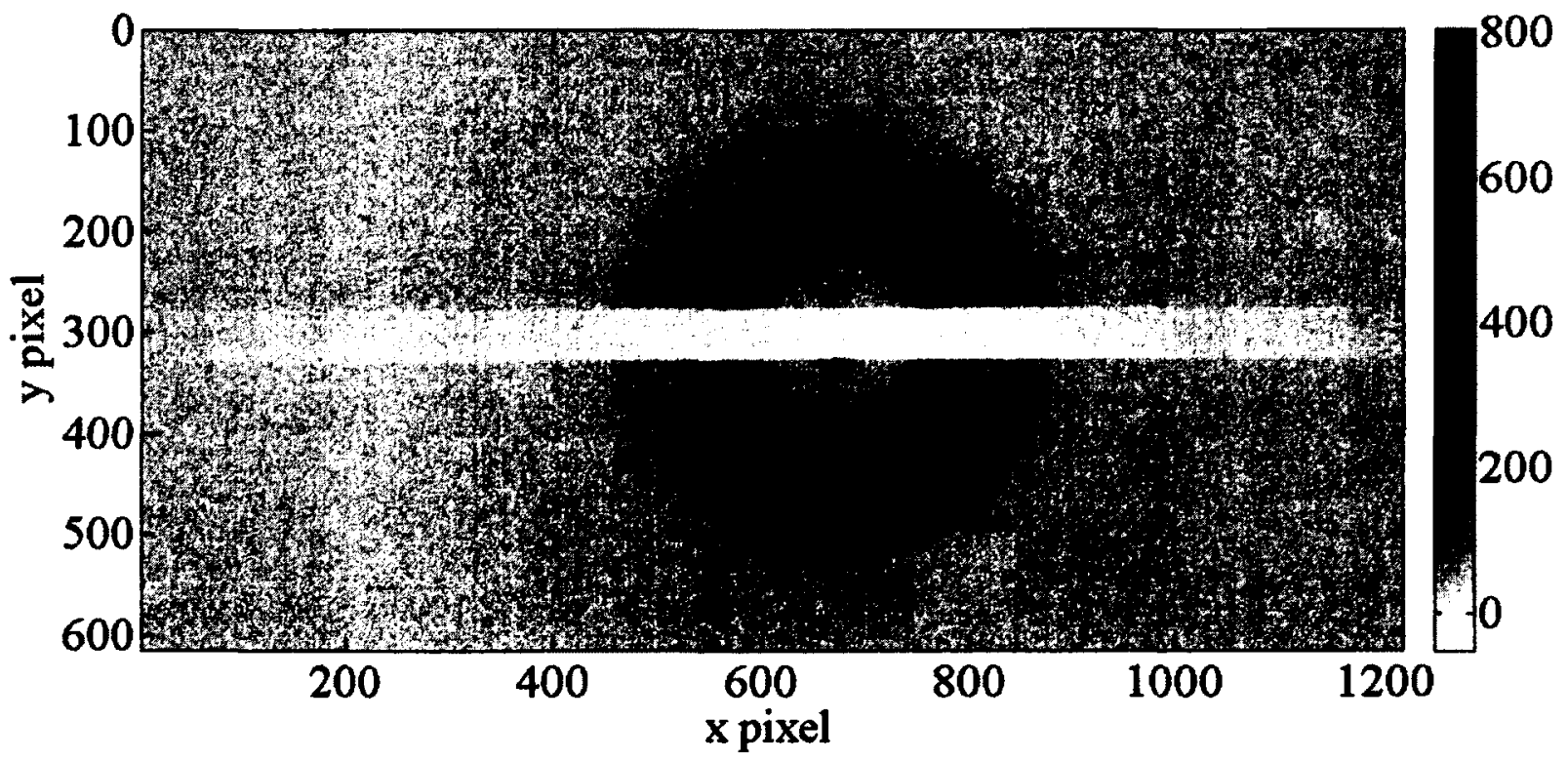

Figure 5.2: Median filtered scatter pattern of pork fat. 


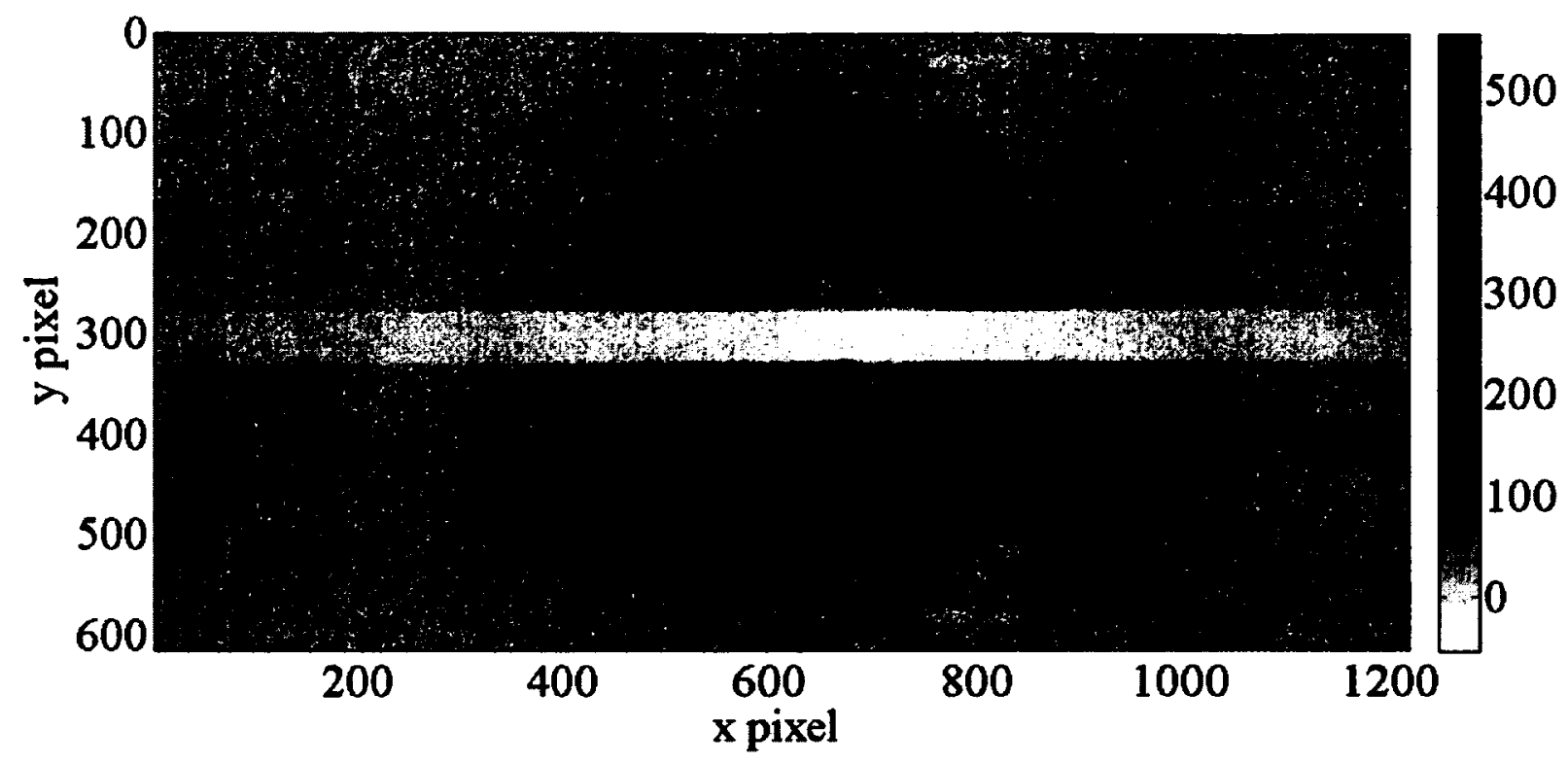

Figure 5.3: Median filtered scatter pattern of pork muscle.

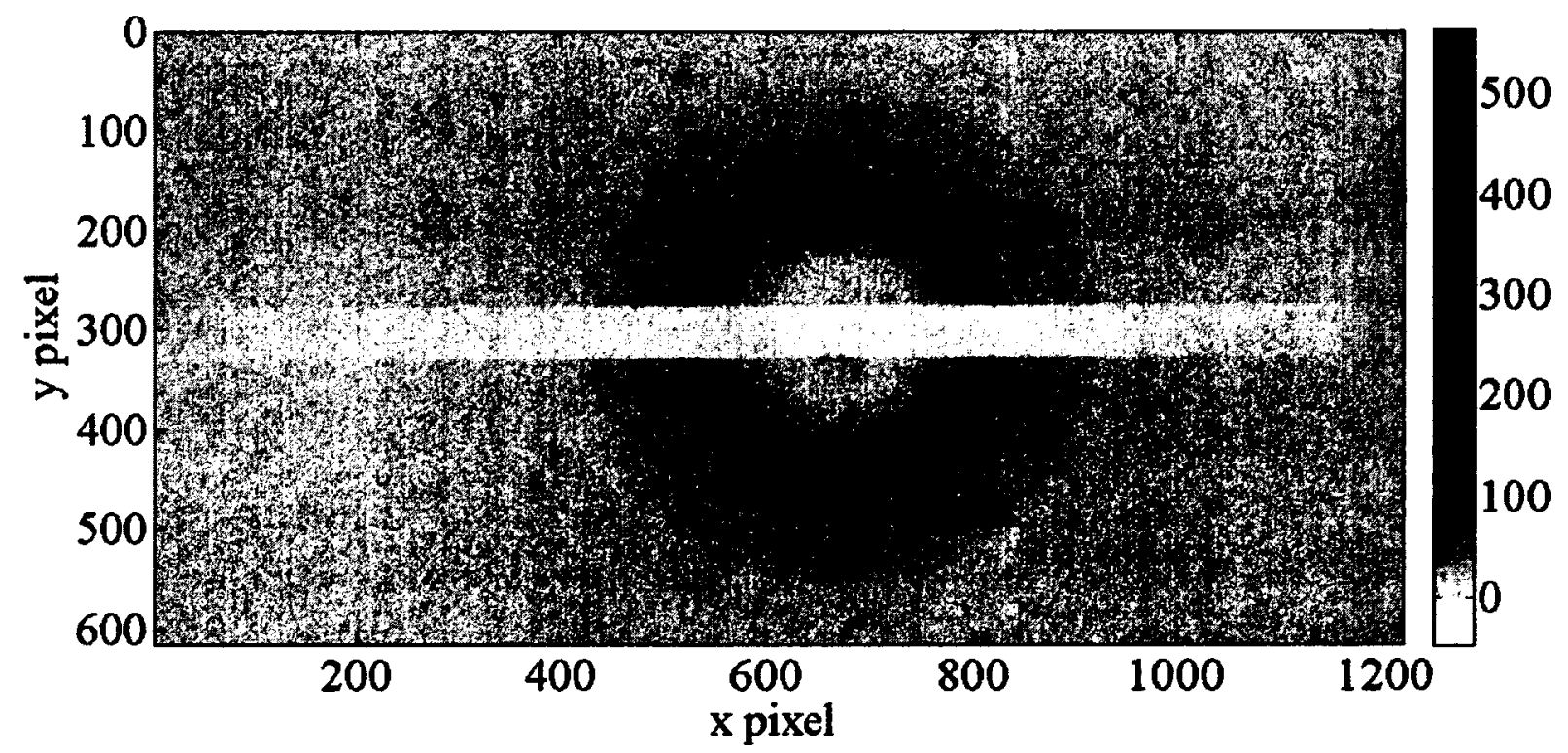

Figure 5.4: Median filtered scatter pattern of pork cortical bone. 


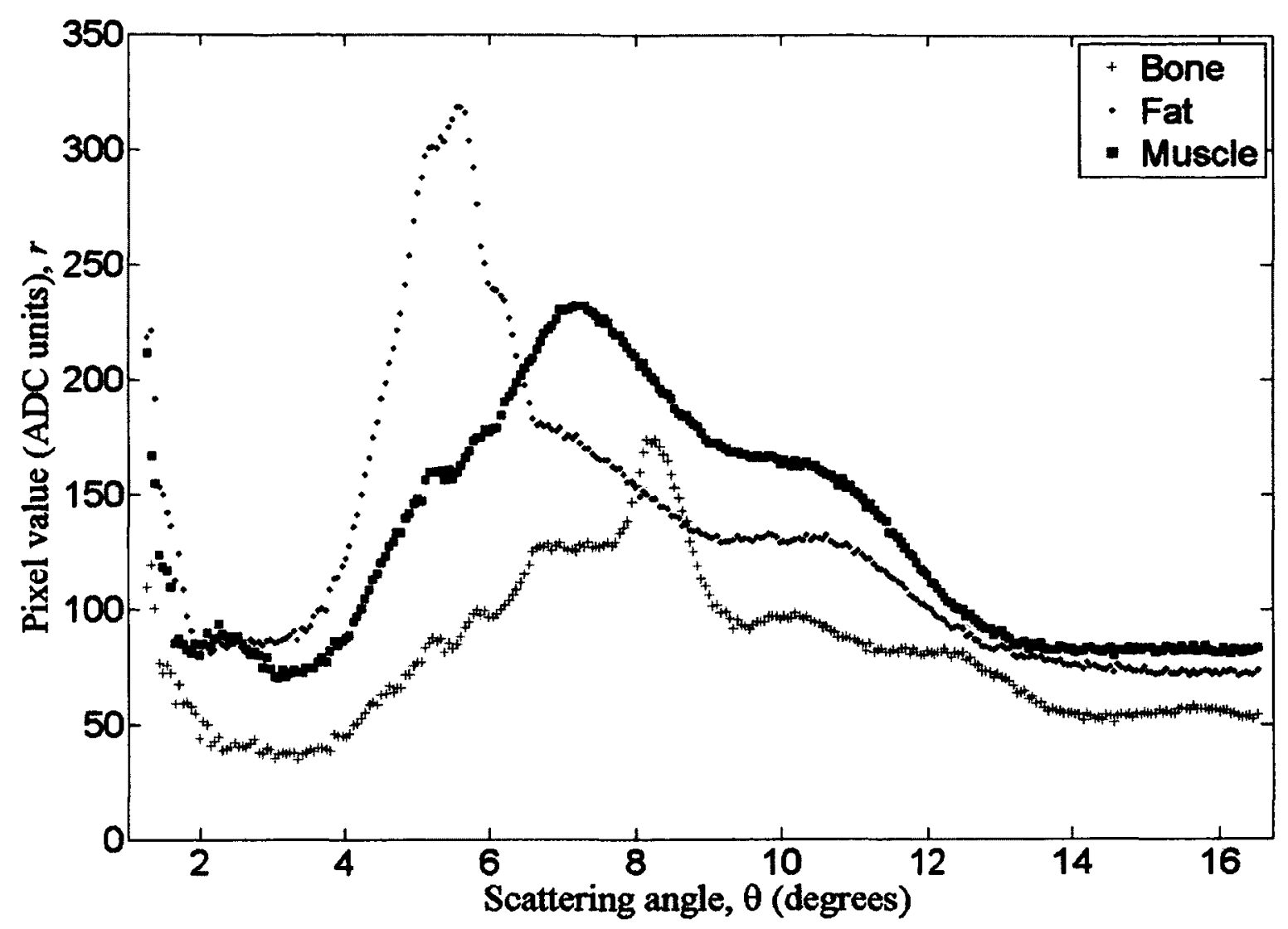

Figure 5.5: Radial profiles of fat, muscle and bone. Certain defect pixels in Figures 5.2 5.4 due to detector damage were excluded in the averaging algorithm.

Figure 5.6 is the primary image for reference of the porcine phantom. Figure 5.7 is the scatter image, acquired simultaneous to Figure 5.6, and was generated by integrating the angular range $3.50^{\circ} \leq \theta \leq 5.57^{\circ}$ which maximizes the fat to muscle contrast, corrected for attenuation. To correct for attenuation the scatter images were divided, pixel-by-pixel, by the primary image. Figure 5.8 is the scatter image generated by integrating over the entire angular range which maximizes contrast of muscle to bone, corrected for attenuation. Note how scatter imaging reveals fat in the lower left region not visible in the primary image but clear from the photograph of the sample. 


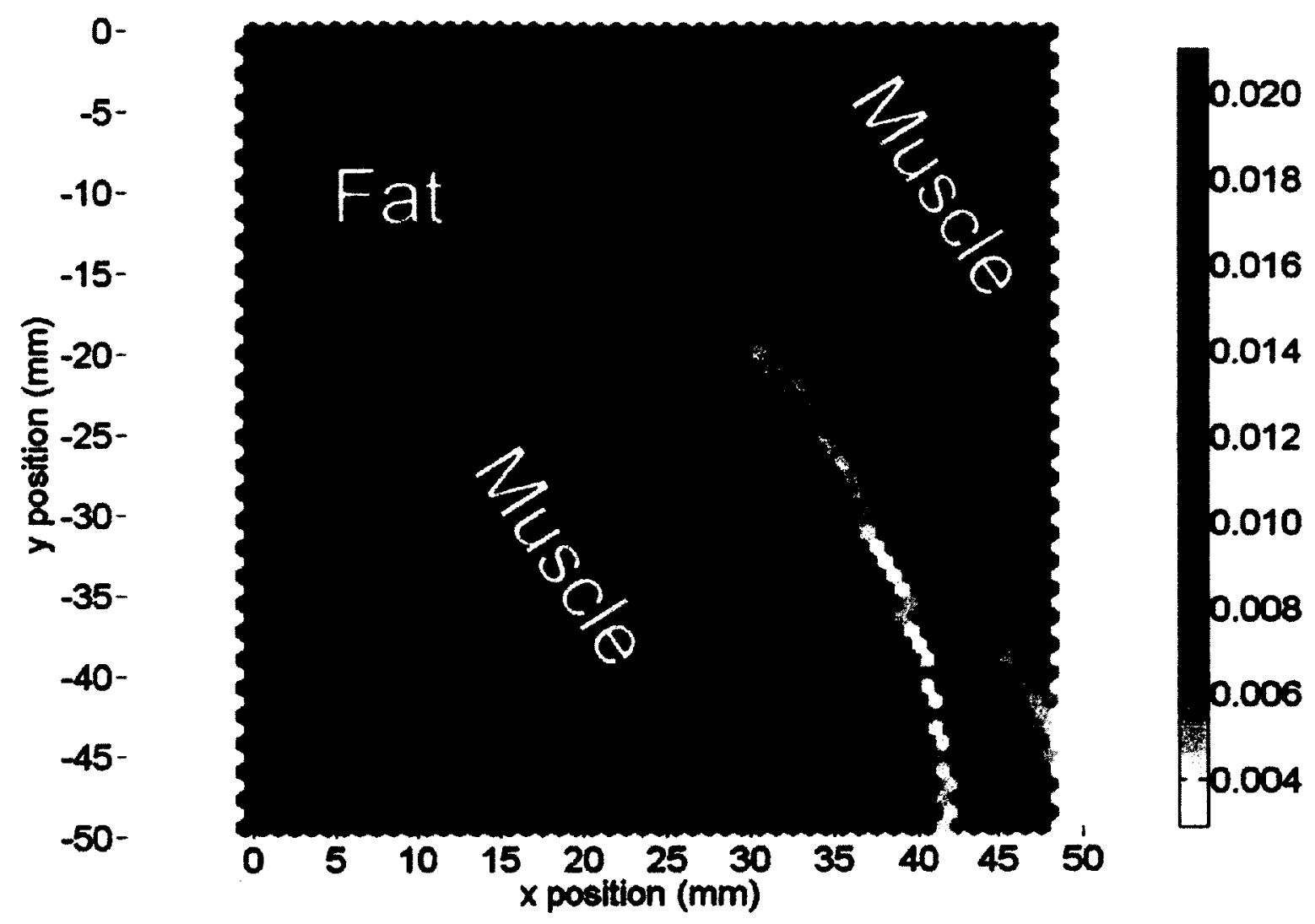

Figure 5.6: Primary image of porcine phantom of Figure 5.1. The data used to make this figure were obtained simultaneously with the data used to make Figure 5.7 and Figure 5.8. Tissue regions have been labeled for illustrative purposes.

The contrast between fat, muscle and bone of both the primary and scatter (corrected for attenuation) single beam images are given in Table 5.2. The angular range which maximizes the contrast between the two materials of interest was used. 


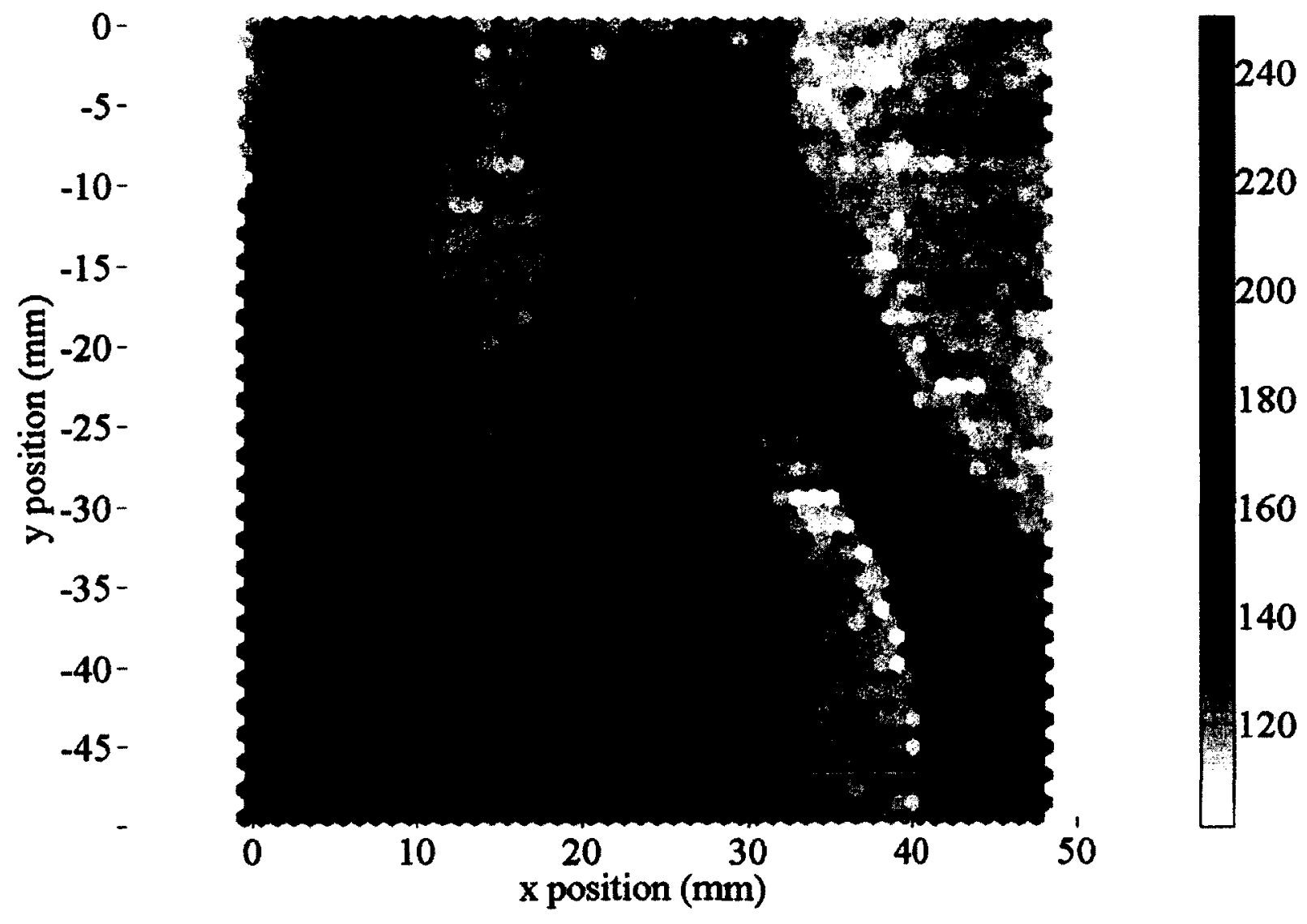

Figure 5.7: Scatter image of porcine phantom, generated by integrating the angular range $3.50^{\circ} \leq \theta \leq 5.57^{\circ}$ which maximizes the fat to muscle contrast, corrected for attenuation. 


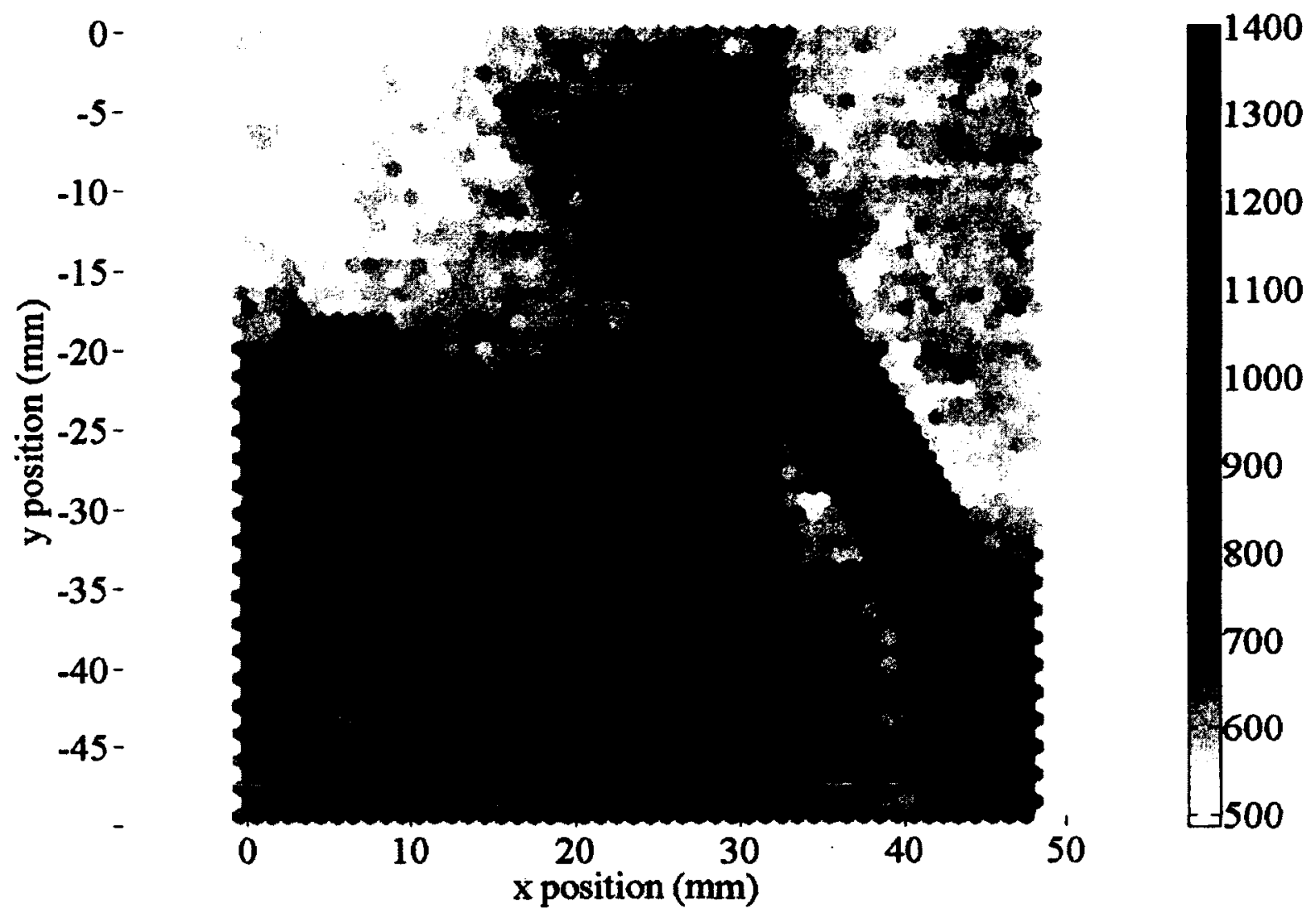

Figure 5.8: Scatter image of porcine phantom, generated by integrating over the entire angular range which maximizes muscle to bone contrast, corrected for attenuation.

Table 5.2: Contrast for single beam measurements for both primary and scatter (corrected for attenuation) images, June 2011 experiment.

\begin{tabular}{|l|l|c|l|c|}
\hline Material 1 & Material 2 & Primary contrast & $\begin{array}{l}\text { Angular range } \\
\text { (degrees) }\end{array}$ & Scatter contrast \\
\hline Fat & Muscle & $0.03 \pm 0.01$ & {$[3.495 .57]$} & $0.41 \pm 0.01$ \\
\hline Fat & Bone & $0.74 \pm 0.02$ & {$[1.328 .52 ;$} & $0.22 \pm 0.02$ \\
& & & $8.8817 .12]$ & \\
\hline Muscle & Bone & $0.77 \pm 0.02$ & {$[1.3217 .12]$} & $0.36 \pm 0.02$ \\
\hline
\end{tabular}


It is clear from Table 5.2 that the scatter soft-tissue contrast is significantly greater than the primary contrast, as predicted by our lab's semianalytic model. ${ }^{28}$ This singlebeam scan, however, took $10 \mathrm{~h}$ to complete which is far too long for any clinical application. Thus we investigated scatter projection imaging using multiple beams.

\subsection{Multi beam}

The multi-beam results are dependent on how well the disentangling algorithm extracts the (theoretically) independent radial profiles. It is reasonable to believe that the disentangling algorithm will perform better with fewer beams, so for this reason a twobeam setup was the first investigation after one beam. For two and three beams the spacing between beams was $32 \mathrm{~mm}$. For five beams the spacing was $16 \mathrm{~mm}$.

Figure 5.9 is a photograph of a phantom which incorporates different plastics, pork bone, and $10 \mathrm{~mm}$ thick cuvettes of pork fat, pork muscle and water on top of a series of $8.4 \mathrm{~mm}$ thick Lucite (polymethyl methacrylate) steps. The total thickness of Lucite ranges from $8.4 \mathrm{~mm}$ to $33.6 \mathrm{~mm}$. Figure 5.10 is the primary $\mathrm{x}$-ray image for comparison, with the materials labeled. Figure 5.11 is the scatter image generated by integrating the angular range $2.00^{\circ} \leq \theta \leq 4.00^{\circ}$. The data of Figure 5.12 were generated by dividing those of Figure 5.11 by Figure 5.10 to correct for attenuation. The data displayed in Figure 5.13 are identical to that of Figure 5.12 but have been windowed and leveled to reduce contrast so that a greater range of signals can be observed.

Figure 5.10, Figure 5.11 and Figure 5.12 have been windowed and leveled so that all three have the contrast of water to acrylic held constant. These two materials were 


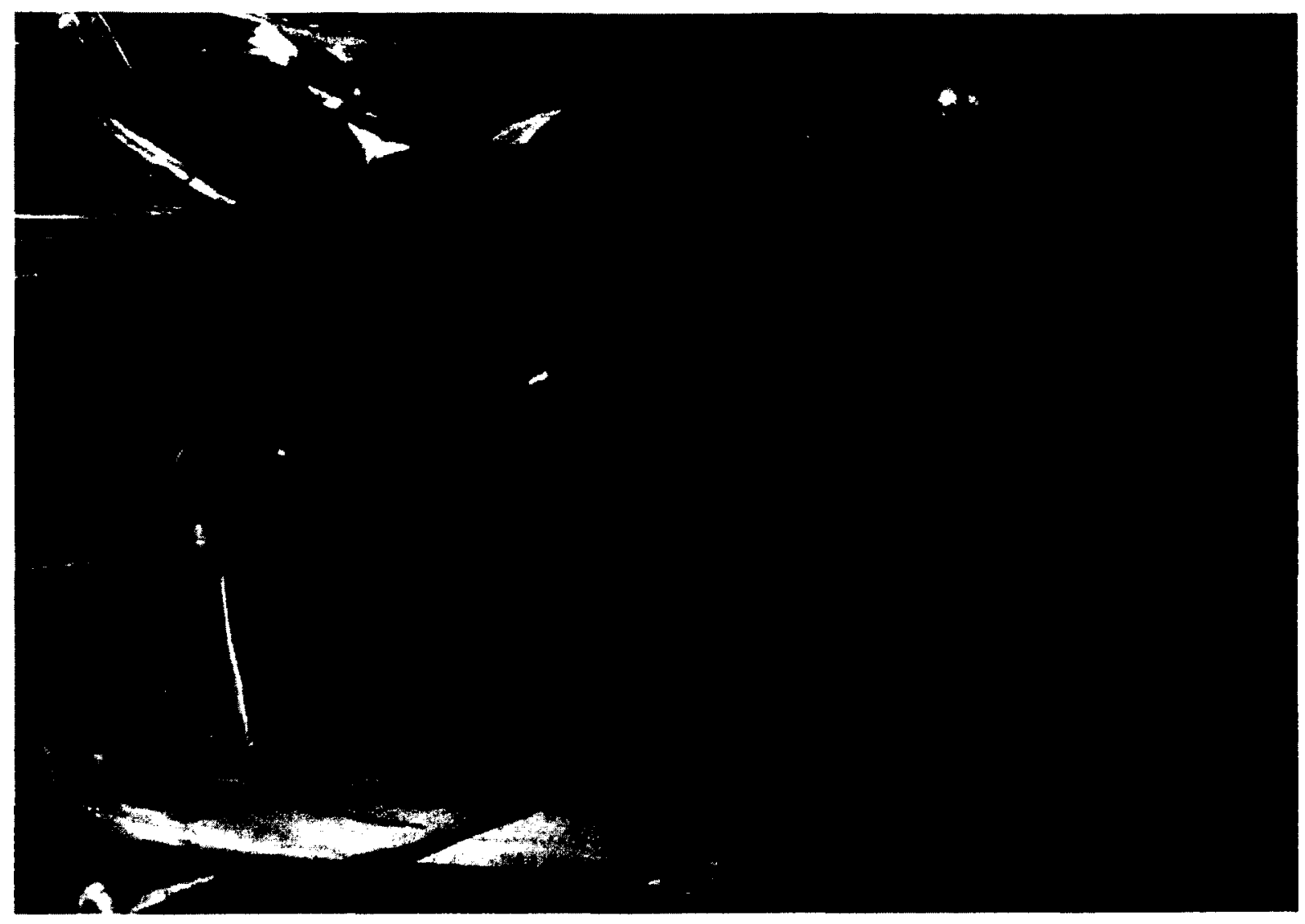

Figure 5.9: Photograph of the tissue/plastic phantom used to generate images shown in Figures $5.10-5.13$. The steps were constructed out of Lucite.

chosen so that all three images would not have a significant portion of the data truncated while still allowing the data to span the entire grayscale. It should be noted that there is substantially greater contrast between differing materials in the scatter image. In particular muscle/fat, polyethylene/Lexan and polystyrene/acrylic appear almost identical in the primary image, while in the scatter image they look substantially different. 


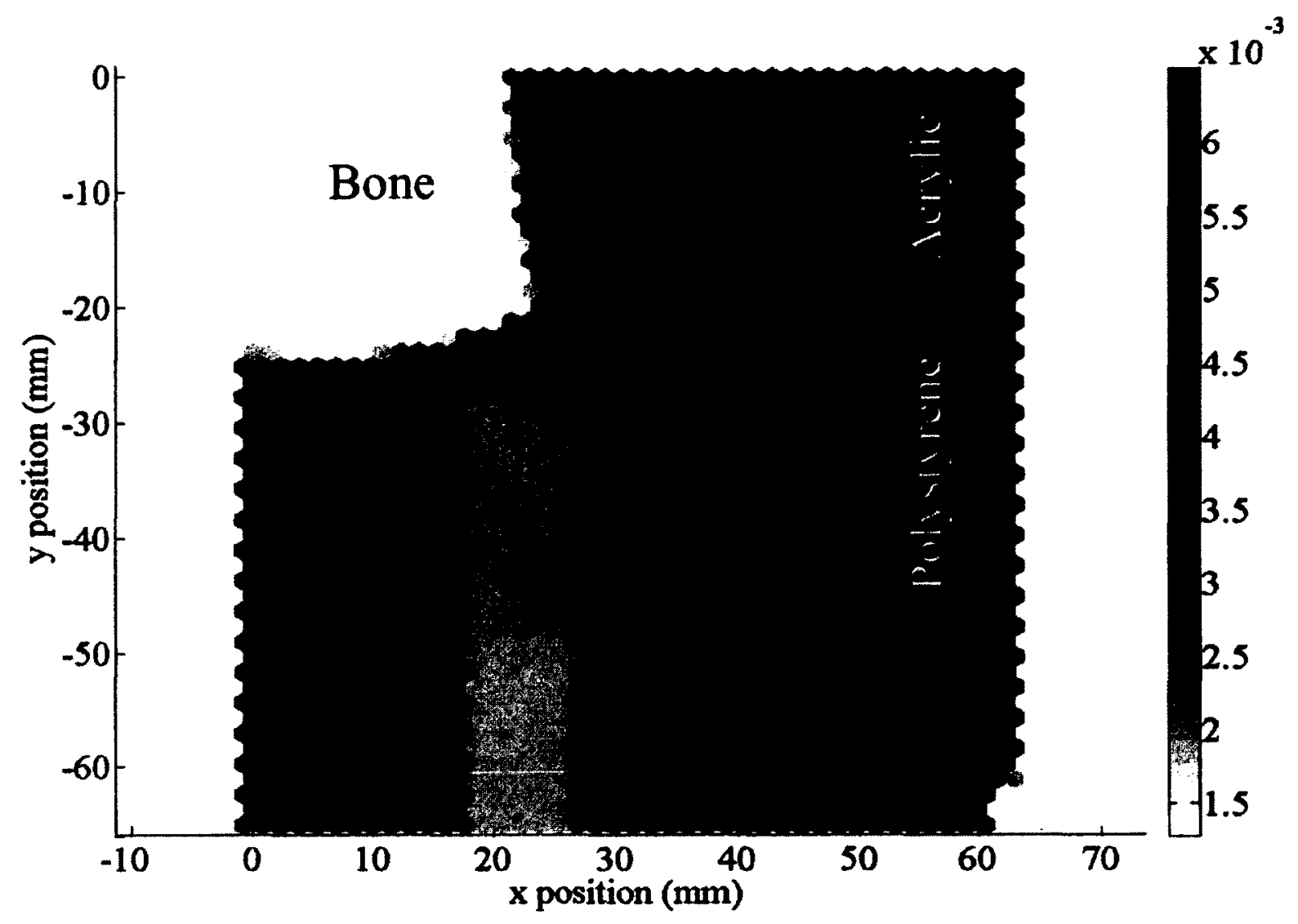

Figure 5.10: Two-beam primary image of tissue/plastic phantom, a photograph of which is shown in Figure 5.9. Individual materials are labeled for identification.

Values of contrast between pairs of materials were calculated and are displayed in Table 5.3; unlike the data displayed in Table 5.2 the ideal angle ranges have not been used to calculate all the contrast values. This is because knowledge of all the materials' form factors through the path of the beam must be known in order to calculate the ideal angle ranges. Not all the materials used have been measured but the energy-dispersive technique developed in our lab ${ }^{29,30}$ is able to do so. With the exception of the highattenuating bone the scatter contrast between pairs of materials is significantly greater than the primary contrast. 


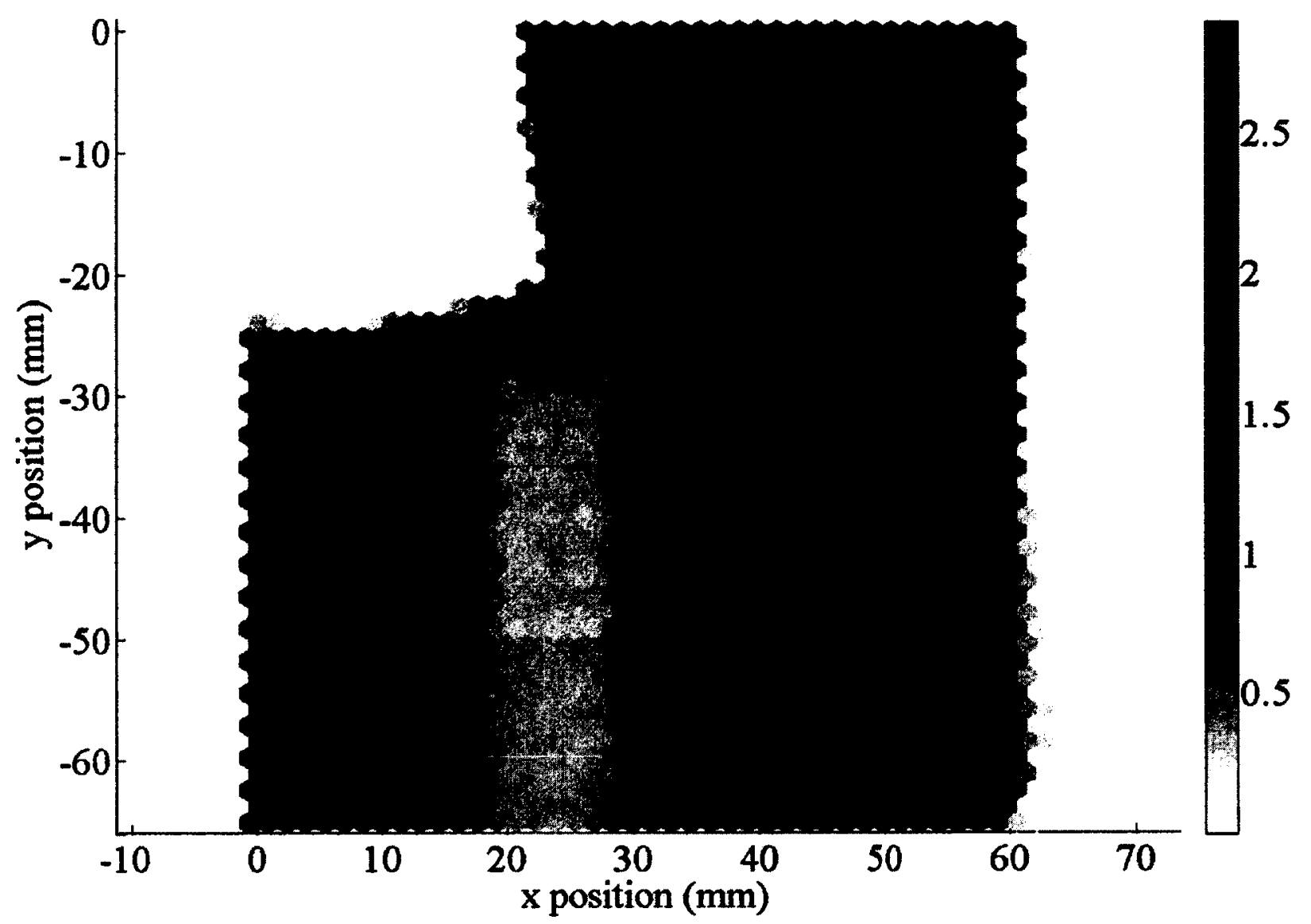

Figure 5.11: Two-beam coherent scatter image generated by integrating the radial profiles over the angular range $2.00^{\circ} \leq \theta \leq 4.00^{\circ}$. 


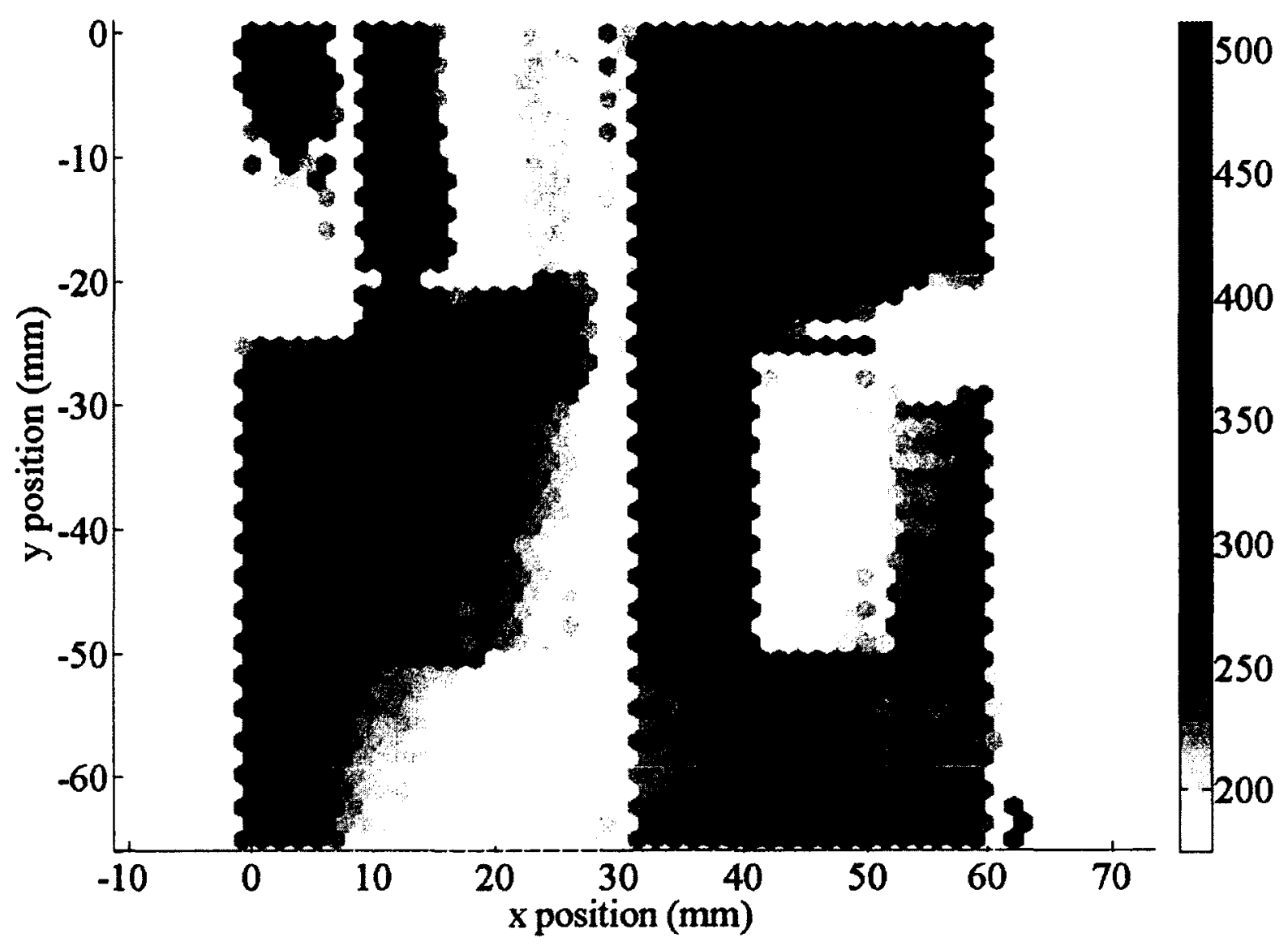

Figure 5.12: Two-beam coherent scatter image divided by the primary image to remove contrast due to attenuation. These are the data displayed in Figure 5.11 divided by the data displayed in Figure 5.10. 


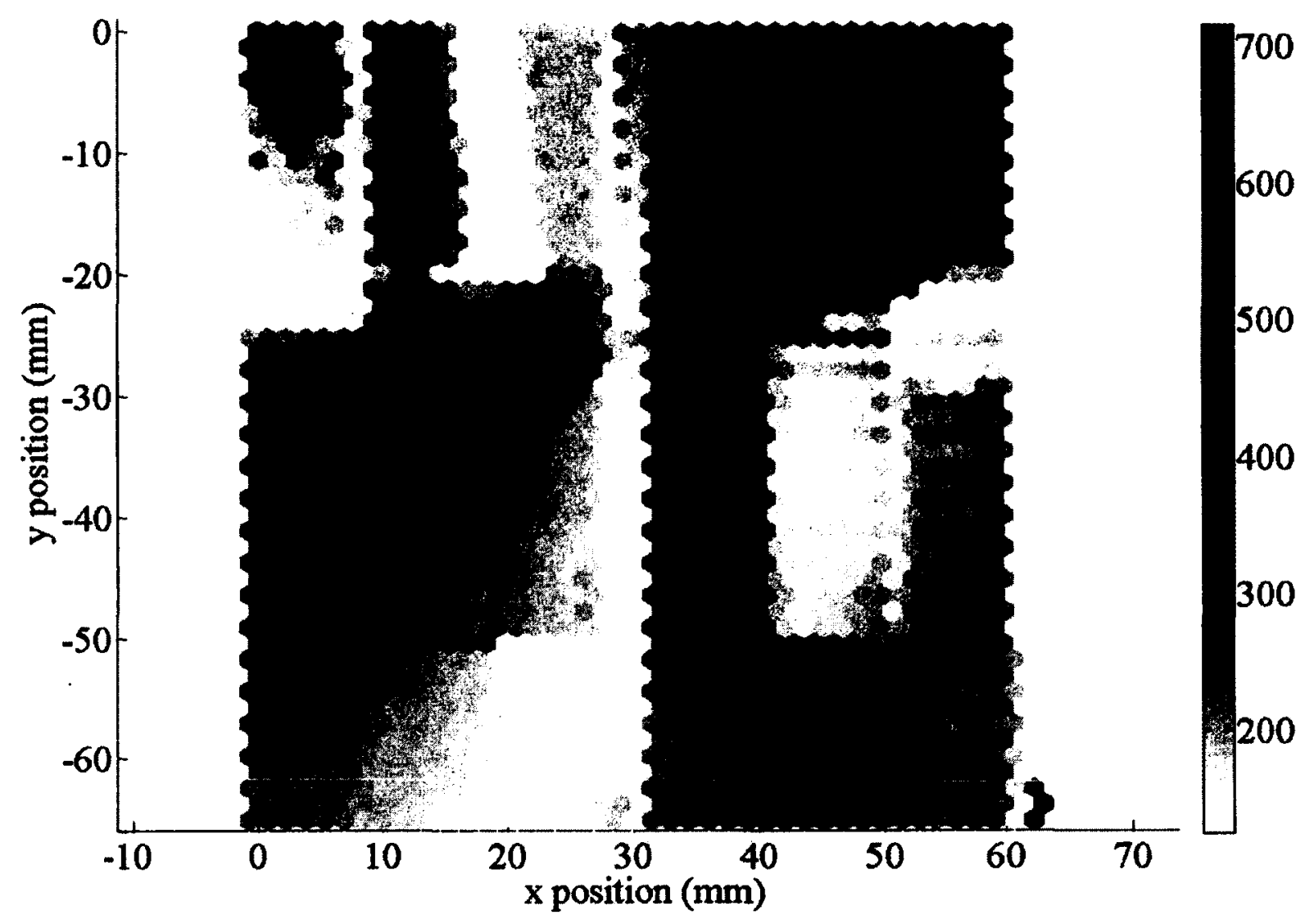

Figure 5.13: Two-beam coherent scatter image divided by the primary image to remove contrast due to attenuation. These are the data displayed in Figure 5.12 windowed and leveled to reduce contrast and display a larger range of values. 
Table 5.3: Contrast between pairs of materials for two-beam measurements for both primary and scatter (corrected for attenuation), images of December 2011. The angular range used for all scatter contrasts was $2.00^{\circ} \leq \theta \leq 4.00^{\circ}$.

\begin{tabular}{|l|l|c|c|}
\hline \multicolumn{1}{|c|}{ Material 1 } & \multicolumn{1}{|c|}{ Material 2 } & Primary contrast & Scatter contrast \\
\hline Polyethylene & Lexan & $0.04 \pm 0.01$ & $0.95 \pm 0.01$ \\
\hline Polystyrene & Acrylic & $0.06 \pm 0.01$ & $0.72 \pm 0.02$ \\
\hline Muscle & Fat & $0.09 \pm 0.01$ & $0.91 \pm 0.02$ \\
\hline Fat & Bone & $1.870 \pm 0.004$ & $0.84 \pm 0.03$ \\
\hline Muscle & Bone & $1.824 \pm 0.004$ & $0.08 \pm 0.04$ \\
\hline
\end{tabular}

From Table 5.3 it is clear that the contrast between these sets of materials from coherently scattered photons is several times that from the primary photons, with the exception of the high-attenuating bone.

A tissue phantom similar to the one shown in Figure 5.1 was also imaged with one and two beams. A photograph of the sample is shown in Figure 5.14. Figure 5.15 is the single beam primary image of the phantom. The dark region on the far right of the image is due to imaging past the boundary of the target (thus there is no attenuation and the signal is large). The strange pattern around the bottom edge of the image is likely due to imaging the sides of the Lucite container. Figure 5.16 is the scatter (maximizing fat to muscle), corrected for attenuation for one-beam. The far right edge is light because we imaged past the boundary of the material (thus there is no target so no scatter). The strange pattern around the edges is again due to imaging the bottom of the container. 


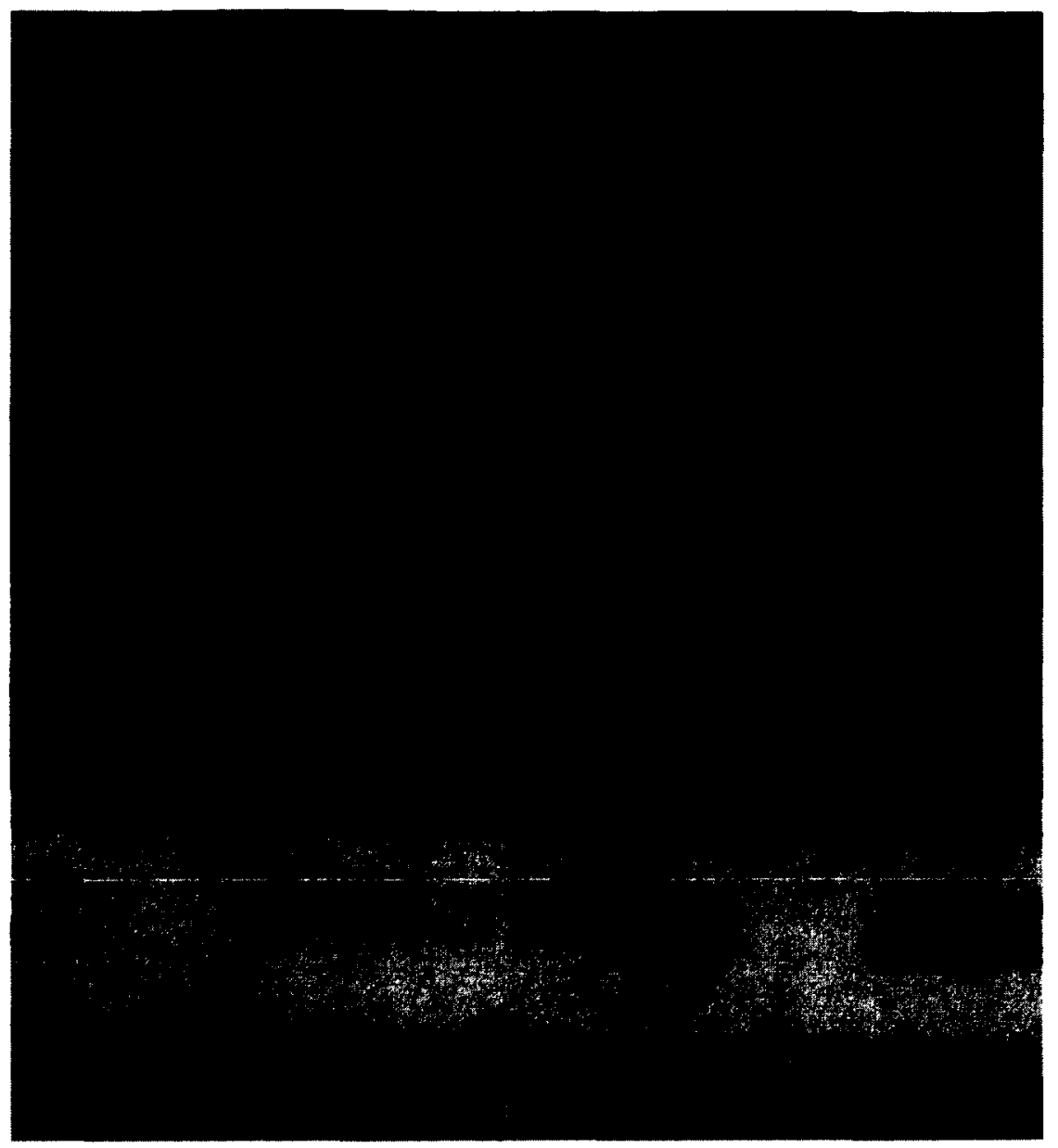

Figure 5.14: Photograph of porcine phantom. Figures 5.15 - 5.18 are primary and scatter images of this sample with various numbers of beams.

Figure 5.17 is the two-beam primary and Figure 5.18 is the two-beam scatter (maximizing fat to muscle), corrected for attenuation. Again there is much greater softtissue contrast in the scatter image when compared to the primary image. 


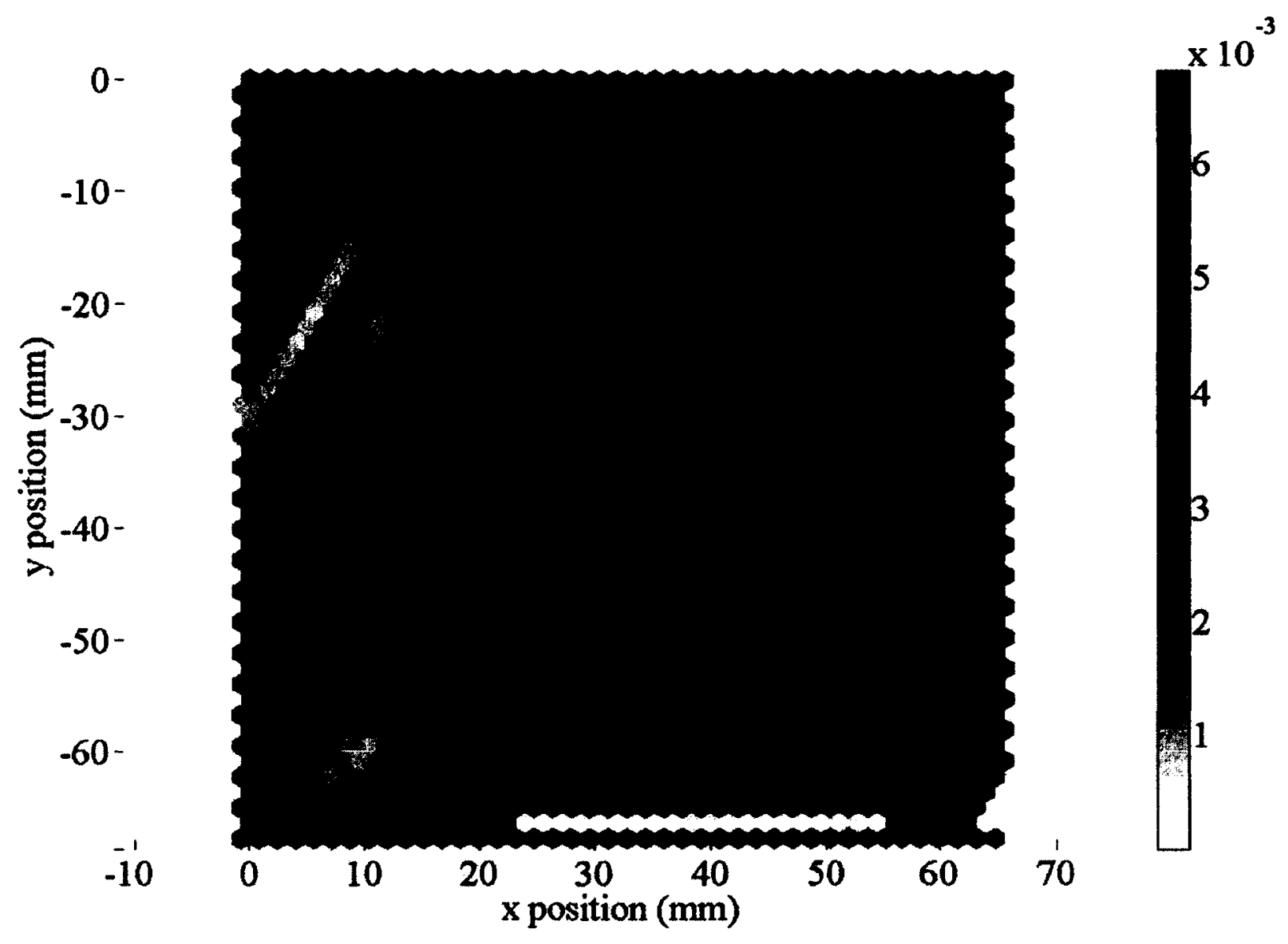

Figure 5.15: Primary image of porcine phantom using a single beam. The strange patterns around the edges of the image are artifacts. 


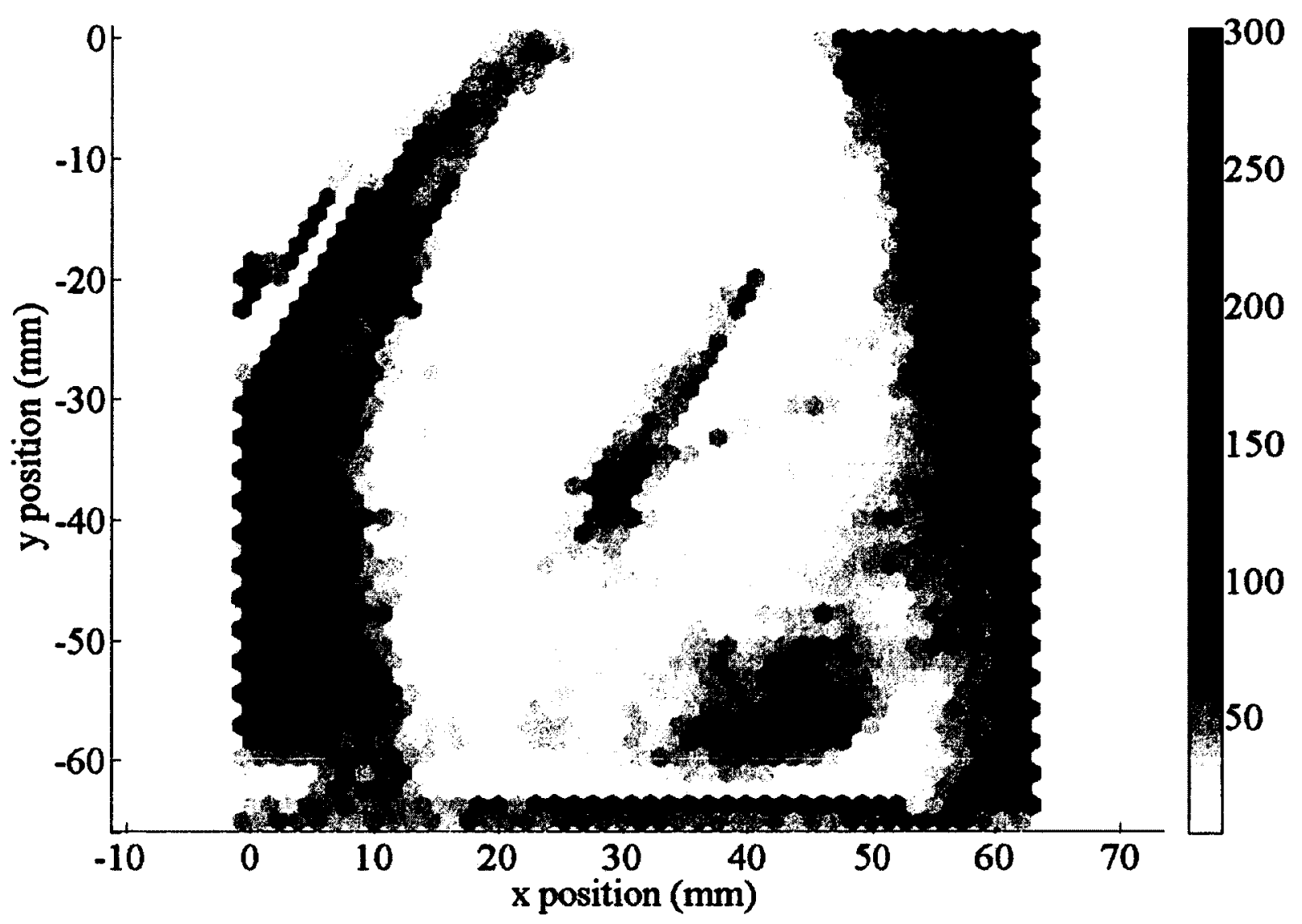

Figure 5.16: Scatter divided by primary image to correct for attenuation using one beam. Contrast of fat to muscle was maximized. The strange patterns around the edges of the image are artifacts. 


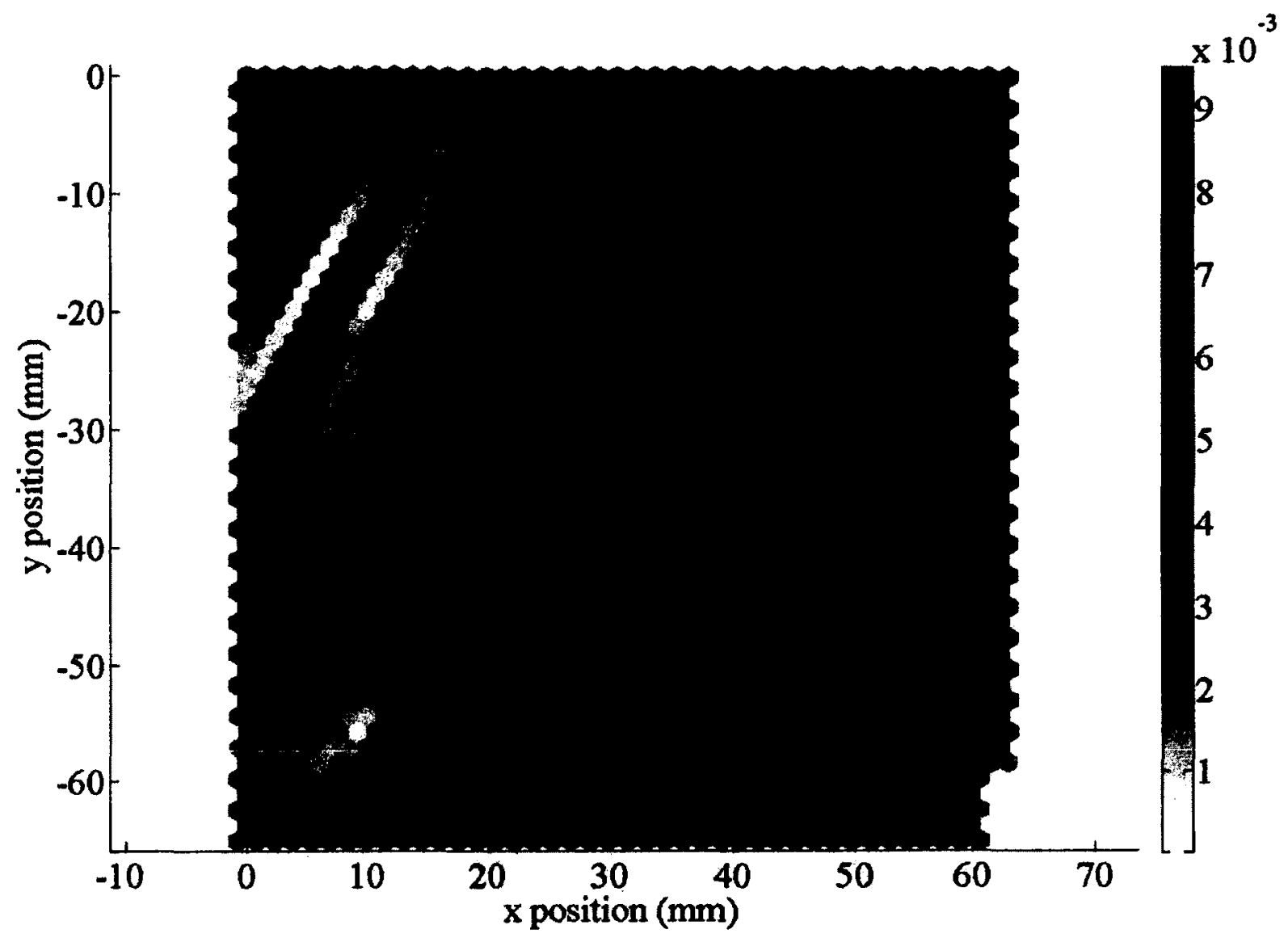

Figure 5.17: Primary image of porcine phantom made using two simultaneous pencil beams.

The contrasts for the one and two-beam measurements of this phantom are given in Table 5.4. The angular ranges for the scatter contrast used were the ones which maximize the contrast between the two materials of interest. Again the scatter contrast for fat to muscle is significantly higher than the primary contrast. It should be mentioned that the uncharacteristically high scatter contrast of fat-bone in the 2 beam image is likely due to a ghosting artifact (due to poor scatter image quality from unwanted background scatter), as it was not this high for any previous or subsequent image. 


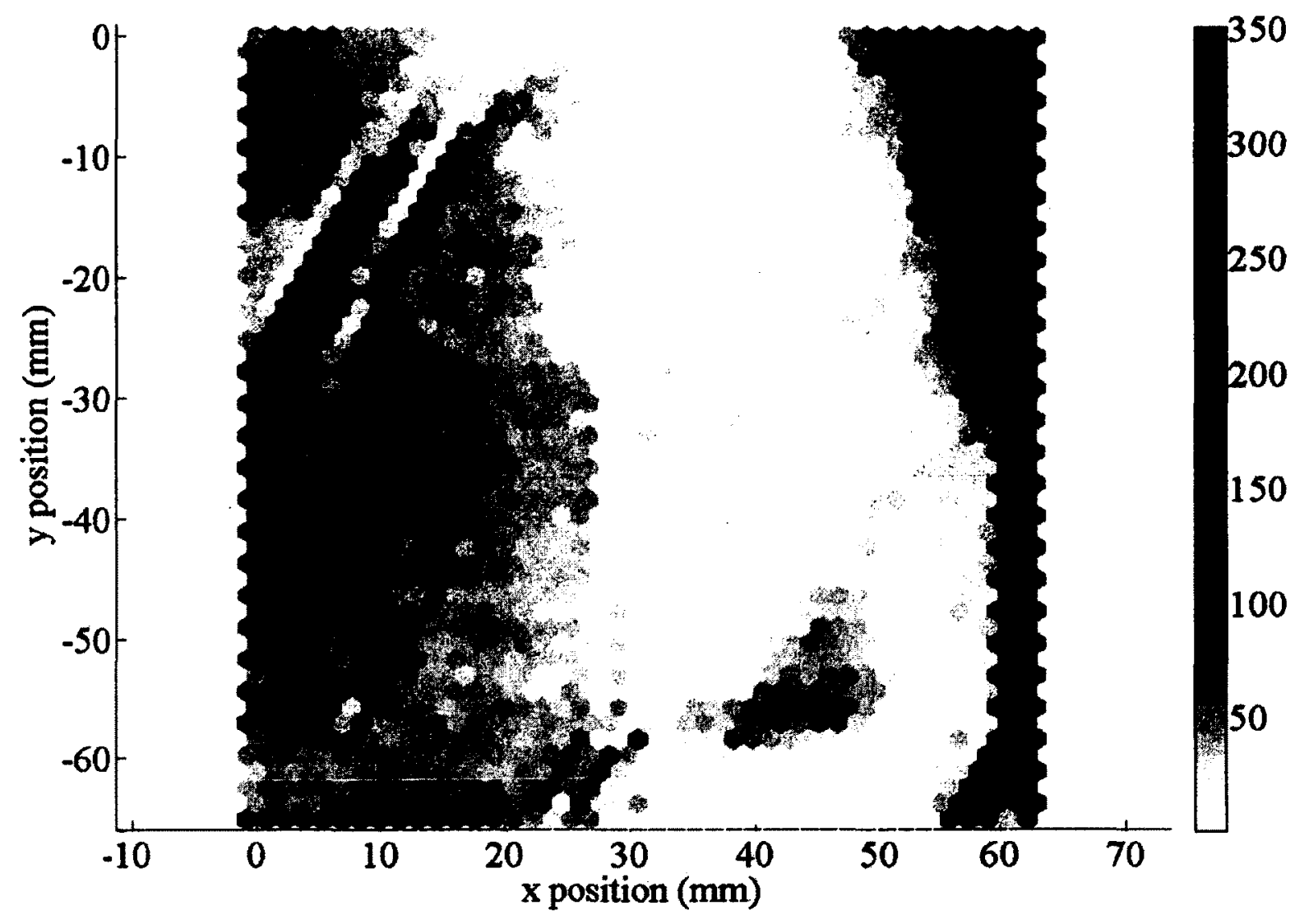

Figure 5.18: Scatter image maximizing contrast between fat and muscle, corrected for attenuation. This image was made using two individual beams. 
Table 5.4: Primary and scatter contrast for both single and double beam measurements of the phantom shown in Figure 5.14, December 2011 experiment.

\begin{tabular}{|c|c|c|c|c|c|c|}
\hline $\begin{array}{l}\text { Material } \\
1\end{array}$ & $\begin{array}{c}\text { Material } \\
2\end{array}$ & $\begin{array}{l}\text { Primary } \\
\text { contrast } \\
\text { (1-beam) }\end{array}$ & $\begin{array}{l}\text { Primary } \\
\text { contrast } \\
\text { (2-beam) }\end{array}$ & $\begin{array}{c}\text { Angular } \\
\text { range } \\
\text { (degrees) }\end{array}$ & $\begin{array}{l}\text { Scatter } \\
\text { contrast } \\
\text { (1-beam) }\end{array}$ & $\begin{array}{l}\text { Scatter } \\
\text { contrast } \\
\text { (2-beam) }\end{array}$ \\
\hline Fat & Muscle & $\begin{array}{l}0.01 \\
\pm 0.04\end{array}$ & $\begin{array}{l}0.02 \\
\pm 0.05\end{array}$ & {$[3.505 .57]$} & $\begin{array}{l}1.76 \\
\pm 0.03\end{array}$ & $1.5 \pm 0.1$ \\
\hline$\overline{\text { Fat }}$ & Bone & $\begin{array}{l}0.71 \\
\pm 0.06\end{array}$ & $\begin{array}{l}0.77 \\
\pm 0.04\end{array}$ & $\begin{array}{l}\left.\begin{array}{ll}0.69 & 8.52 \\
8.88 & 15.32\end{array}\right] \\
\end{array}$ & $\begin{array}{l}0.59 \\
\pm 0.05\end{array}$ & $\begin{array}{l}1.02 \\
\pm 0.03\end{array}$ \\
\hline Muscle & Bone & $\begin{array}{l}0.70 \\
\pm 0.04\end{array}$ & $\begin{array}{l}0.75 \\
\pm 0.02\end{array}$ & {$\left[\begin{array}{lll}0.69 & 15.32\end{array}\right]$} & $\begin{array}{l}0.31 \\
\pm 0.05\end{array}$ & $0.3 \pm 0.1$ \\
\hline
\end{tabular}

There were several detector artifacts in this experiment (December 2011) which we focused on removing for the subsequent experiment (May 2012). Additionally a clear left-right boundary artifact is visible in Figure 5.18 possibly due to a mis-alignment in beams. For the May 2012 experiment we also we increased the number of beams to 5 .

A similar porcine phantom shown in Figure 5.19 was imaged using 1, 3 and 5 beams. Figure 5.20 is the 1 -beam scatter image integrated over $3.50^{\circ}$ to $5.57^{\circ}$, maximizing the contrast between fat and muscle. Figure 5.21 is the 3-beam muscle-fat scatter image. Again, there is significant soft-tissue contrast. The 1-beam primary and scatter images corrected for attenuation cannot be made because photodiode preamplifier \#1 went offline half-way through the five-beam scan. Figure 5.22 is the primary image of the porcine phantom using five beams. The bottom left portion of the image is missing 
due to the photodiode preamplifier. The bone shows up very clearly in this image but fat and muscle cannot be distinguished. Figure 5.23 is the scatter image generated by integrating the radial profile over the entire angular range; this maximizes the contrast between bone and muscle. The bone shows up much clearer in this image than the other scatter images, although the primary is still superior for distinguishing between bone and other tissues. The 5-beam scatter image took the MATLAB code $87 \mathrm{~min}$ to run on an Intel@ Core ${ }^{\mathrm{TM}}$ i7-2600 CPU $3.4 \mathrm{GHz}$ 4-core processor. A significant performance increase could be obtained by compiling the program in C or FORTRAN.

Figure 5.24 is the five-beam scatter image integrated over $3.50^{\circ}$ to $5.57^{\circ}$, maximizing the contrast between fat and muscle. Figure 5.25 is the fat-muscle scatter image, corrected for attenuation; the data displayed in Figure 5.25 are the data shown in Figure 5.24 divided pixel-by-pixel by the data shown in Figure 5.22. The fat-cap on the left can be easily distinguished from the surrounding muscle, whereas this cannot be done for the primary image (Figure 5.22).

Table 5.5 gives the calculated contrasts between the three tissues for both primary and scatter 5-beam images. The angular ranges for the scatter contrast used were the ones which maximize the contrast between the two materials of interest. The areas are $3 \times 3$ groups of pixels deemed to be a specific tissue; the pixels used are outlined in Figure 5.22 for display. The middle-left portion is fat, the centre portion is muscle and the top right group of pixels is bone. The soft-tissue contrast is much greater for the scatter image. 
Table 5.5: Contrast for five-beam measurements for both primary and scatter (corrected for attenuation) images, May 2012 experiment.

\begin{tabular}{|l|l|l|l|c|}
\hline Material 1 & Material 2 & Primary & Angular range (degrees) & Scatter \\
& & contrast & & contrast \\
\hline Fat & Muscle & $0.10 \pm 0.01$ & {$[3.505 .57]$} & $1.16 \pm 0.03$ \\
\hline Fat & Bone & $0.92 \pm 0.04$ & {$[1.058 .52 ; 8.8814 .83]$} & $0.67 \pm 0.03$ \\
\hline Muscle & Bone & $0.84 \pm 0.04$ & {$[1.0514 .83]$} & $0.78 \pm 0.03$ \\
\hline
\end{tabular}

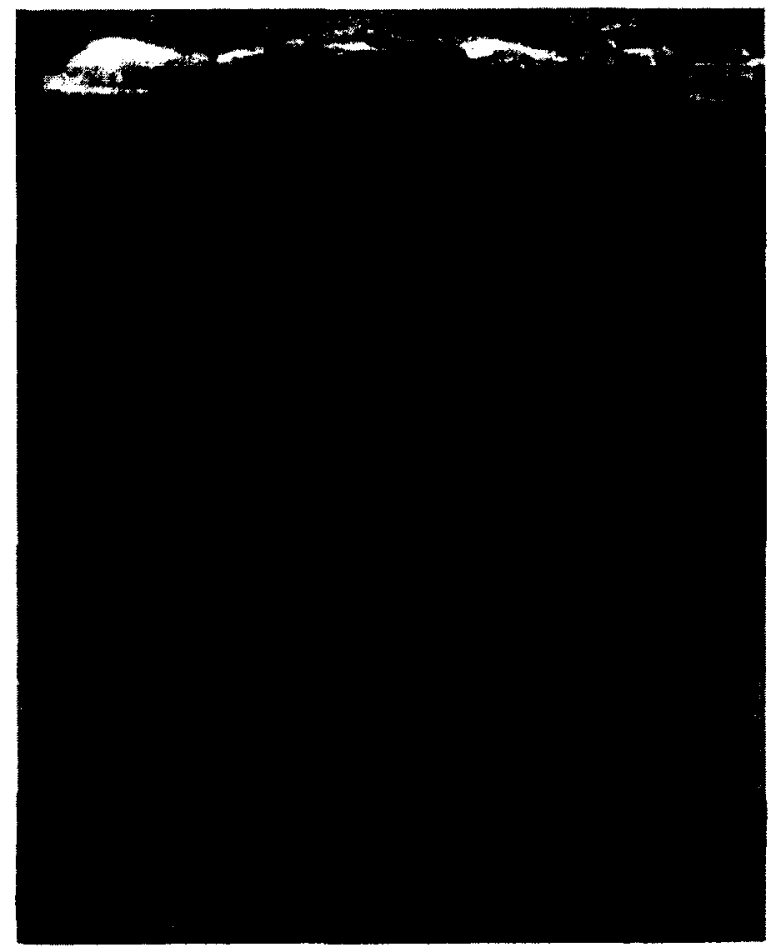

Figure 5.19: Photograph of porcine phantom for the experiments of May 2012. Figures $5.20-5.25$ are primary and scatter images of this sample with various numbers of beams. 


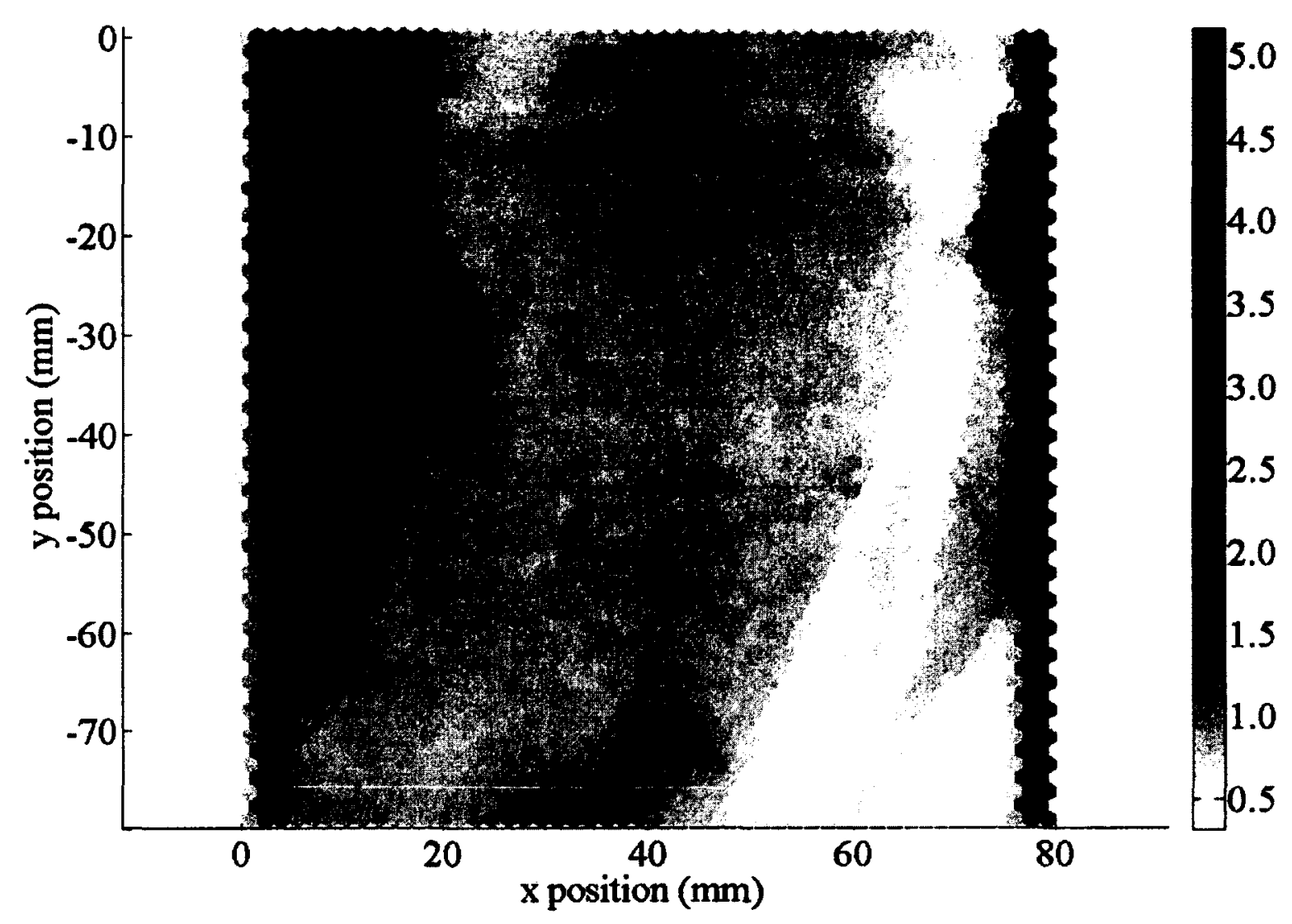

Figure 5.20: One-beam scatter image integrated over $3.50^{\circ}$ to $5.57^{\circ}$. This maximizes the contrast between fat and muscle.

From Table 5.5 it is clear that the soft-tissue contrast is significantly greater for scatter than primary. The one and three beam data cannot be compared due to the lack of data from the photodiode going offline. 


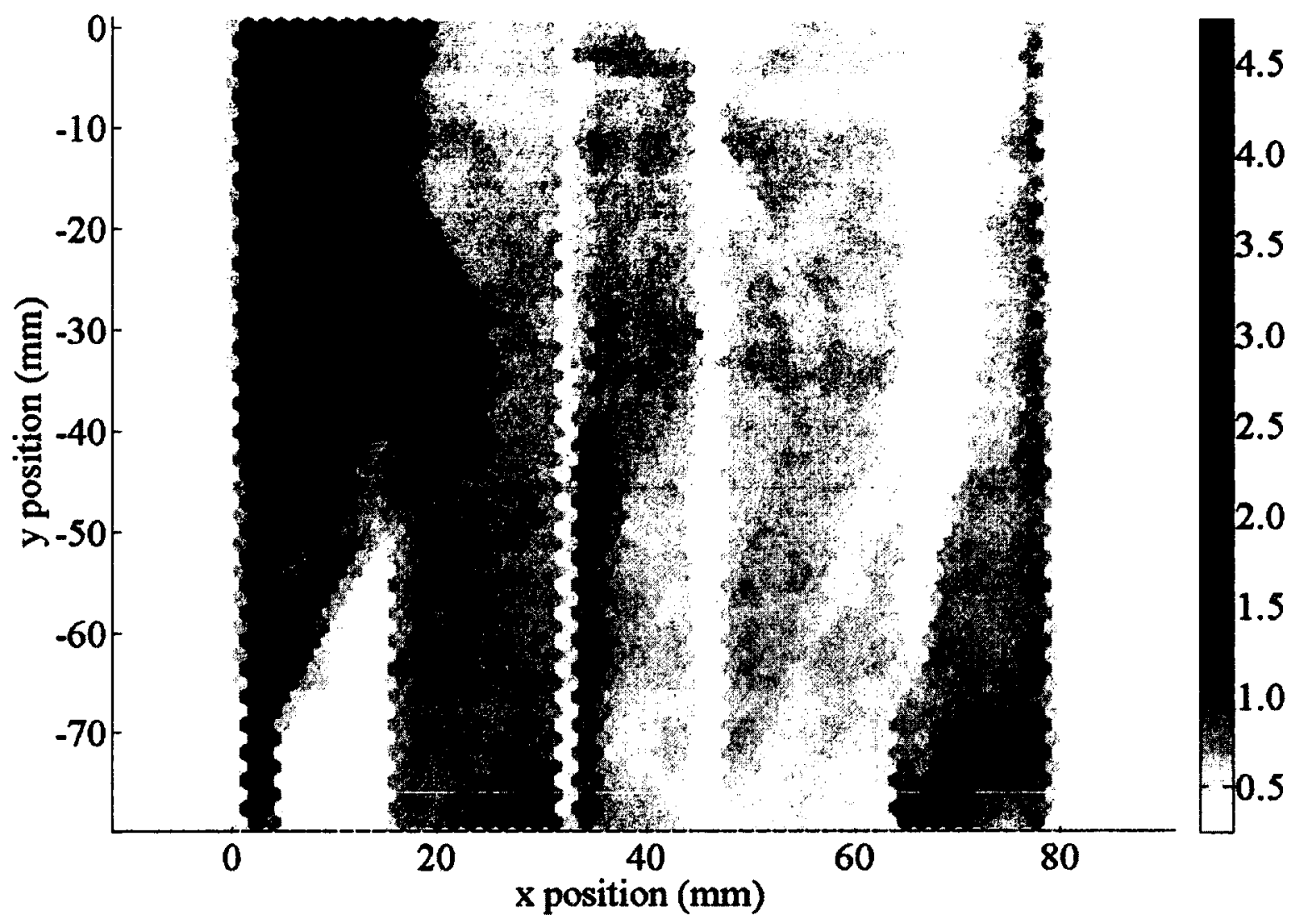

Figure 5.21: Three-beam scatter image integrated over $3.50^{\circ}$ to $5.57^{\circ}$. This is the threebeam equivalent of Figure 5.20 . 


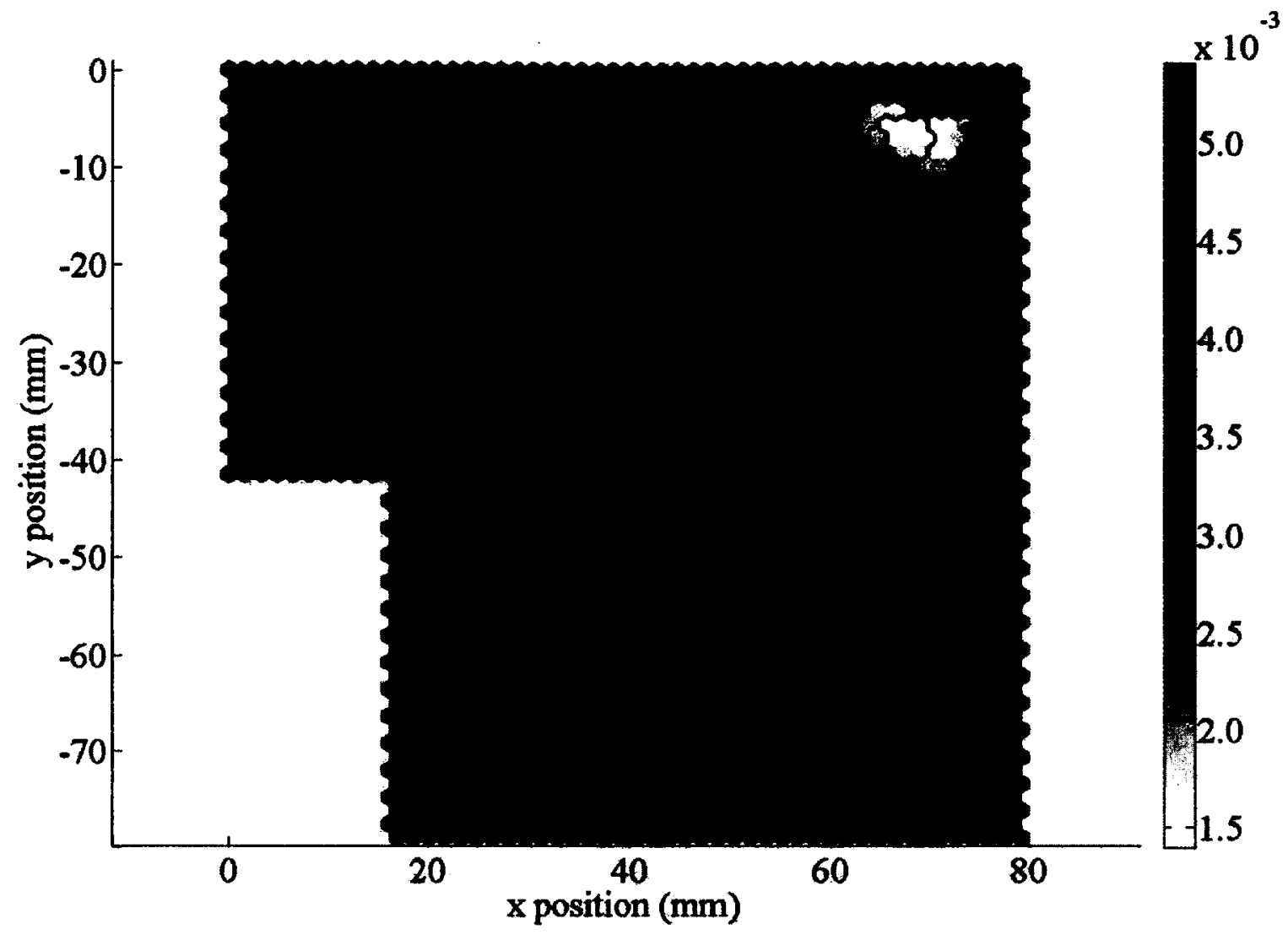

Figure 5.22: Five-beam primary image of porcine phantom. The bottom left corner of image is missing due to photodiode preamplifier \#1 going offline. The three 9-pixel regions used to calculate the contrast values in Table 5.5 are outlined (from left to right: fat, muscle, bone). 


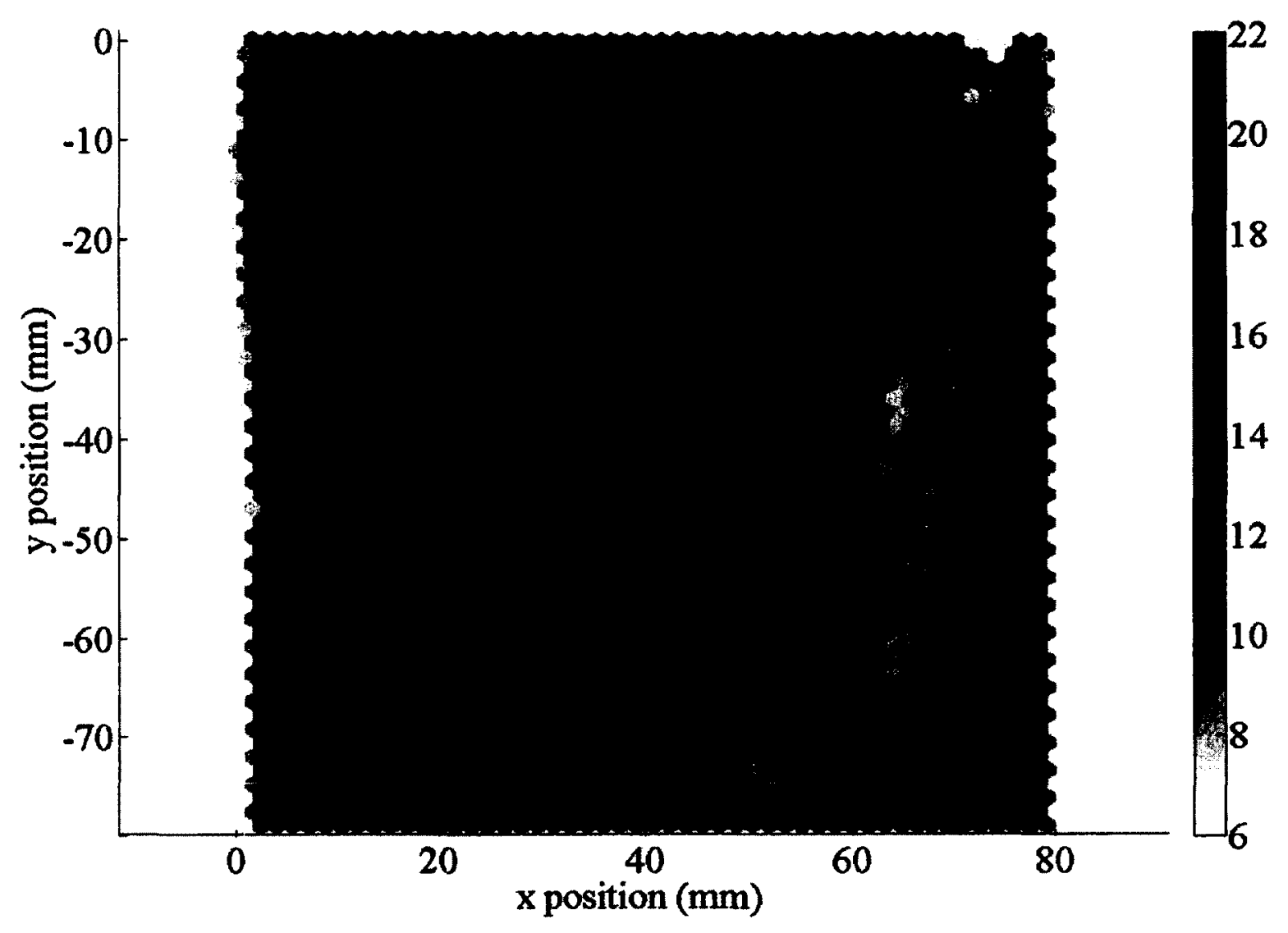

Figure 5.23: Five-beam scatter image integrated over the whole angular range. This maximizes the contrast between muscle and bone. 


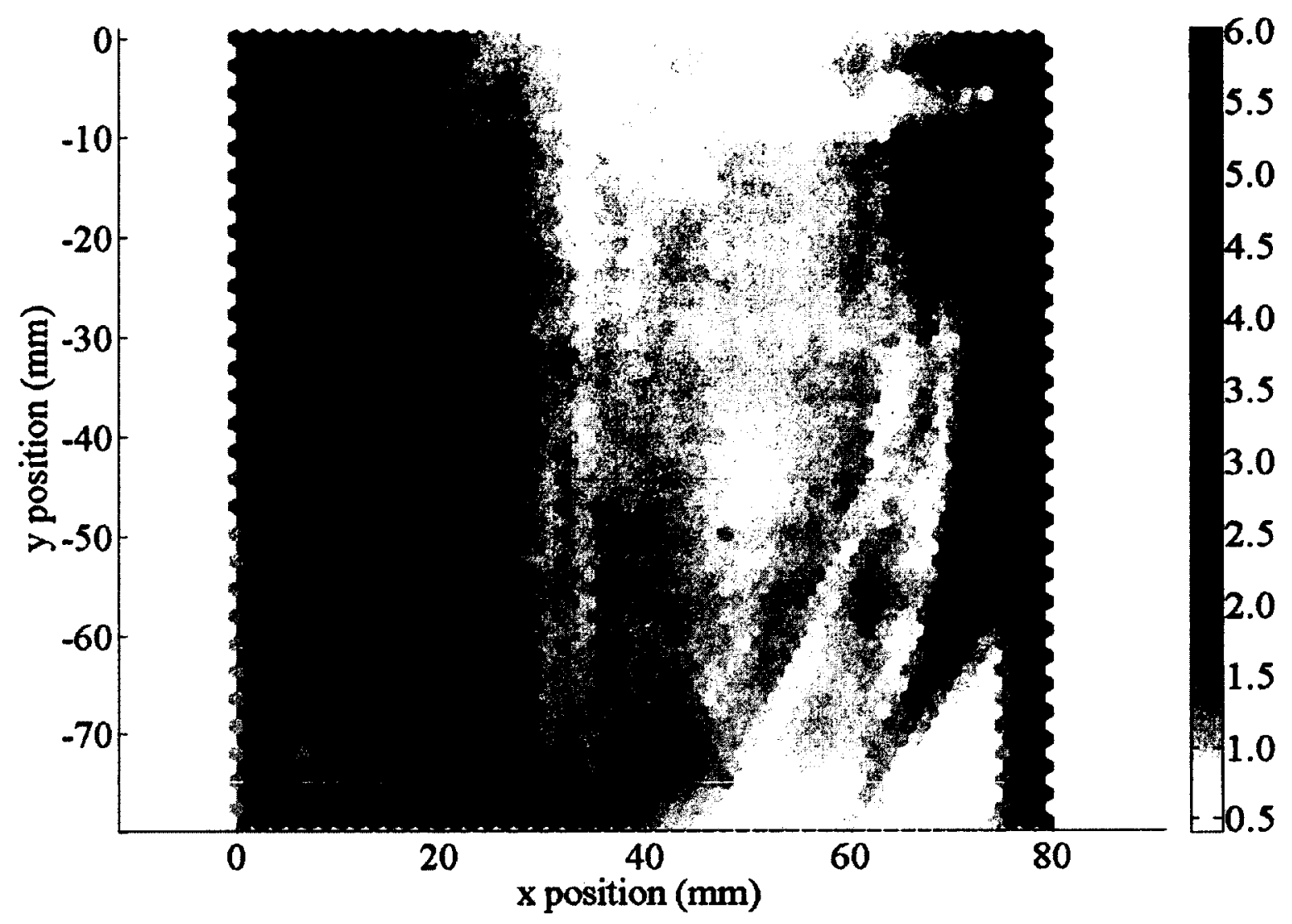

Figure 5.24: Five-beam scatter image integrated over $3.50^{\circ}$ to $5.57^{\circ}$. This is the fivebeam equivalent of Figure 5.20 . 


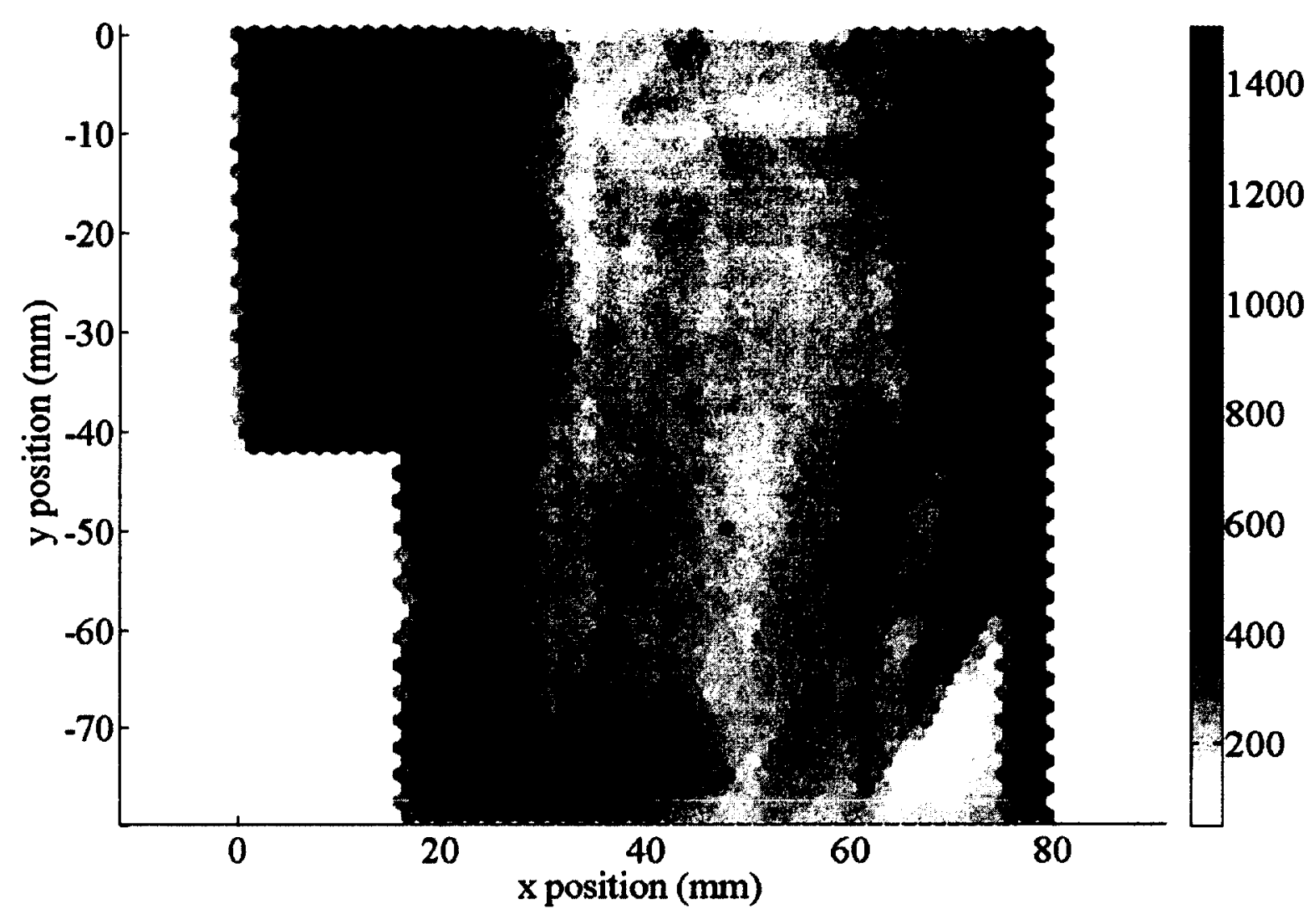

Figure 5.25: Five-beam scatter image maximizing fat to muscle contrast, corrected for attenuation. The data displayed here are the data shown in Figure 5.24 divided (pixel-bypixel) by the data of Figure 5.22 .

From comparing Figure 5.21 and Figure 5.24 to Figure 5.20 it is clear that while the disentangling algorithm does an adequate job, there are still ghosting artifacts that must be removed. In particular ghosting artifacts appear more strongly when one of the beams is of very high or very low intensity. They appear more strongly in the 3-beam image (Figure 5.21) because one beam went past the boundaries of the sample (thus the scatter signal was essentially zero for one of the beams). For the three-beam image the first beam generated the pixels of $x$ position 0 to $31 \mathrm{~mm}$, the second beam generated the pixels from $32 \mathrm{~mm}$ to $63 \mathrm{~mm}$, and the third beam went from $64 \mathrm{~mm}$ to $95 \mathrm{~mm}$. The right portion of the image (after $80 \mathrm{~mm}$ ) has been truncated to match the boundary of the 
sample, otherwise non-physical ghosting artifacts appear. A better disentanglement algorithm would help rectify the problem of ghosting when one beam is zero, and will be proposed in Section 6.2.

It should be mentioned that the fan-beam from the monochromator is not perfectly planar. On average there is about a millimeter horizontal difference from one beam to the next. Thus, although the beams line up horizontally at the boundary, one beam is actually probing a slightly different vertical location than its adjacent beam, resulting in some noticeable discontinuities in the multi-beam images (Figures 5.21 - 5.25). Although these artifacts exist the 1-beam scan took 5 times as long (over 4 hours in total) to complete which is far too long for any practical clinical applications. A possible way to reduce this is explained in Section 6.3.3.

\subsection{Kerma}

The air kerma was measured using OSLDs (see Section 3.5) for the five-beam configuration for the May 2012 experiment. The OSLDs were taped to Styrofoam thus the measurement was close to free in air. Table 5.6 is the number of read-out photon counts. Three corrections were necessary to find the kerma for the images. First, because the calibration was done exposing the entire area of the OSLD and the synchrotron beam does not cover the entire area of the OSLD, we scaled the air kerma by the ratio of the OSLD area $\left(A_{D}\right)$ to the average beam area $\left(A_{b}\right)$ to give the actual kerma within one of the pencil beams. Second, since with our imaging setup not all of the object is irradiated, i.e., the irradiation geometry is an array of beams separated by gaps, we scale the data to give the equivalent kerma for uniform object irradiation by multiplying by the ratio of the 
beam area $\left(A_{b}\right)$ to the pixel area $\left(A_{p}\right)$. This is so we obtain the area-averaged kerma for the image, which is a value we can use to compare to traditional images. Third, the air kerma must be scaled by the ratio of the pixel beam-time $\left(t_{p}=0.99 \mathrm{~s}\right)$ to the OSLD measurement time $\left(t_{m}=3.3 \mathrm{~s}\right)$. The area-averaged air kerma for the image is

$$
K_{a i r}^{\prime}=K_{a i r}\left(\frac{A_{D}}{A_{b}}\right)\left(\frac{A_{b}}{A_{p}}\right) \frac{t_{p}}{t_{m}}=K_{a i r}\left(\frac{A_{D}}{A_{p}}\right) \frac{t_{p}}{t_{m}}
$$

where the prime is used to indicate that the air kerma is averaged over the image.

Table 5.6: Number of read-out photons from the OSLDs exposed at the CLS for $3.3 \mathrm{~s}$.

\begin{tabular}{|l|c|c|c|c|c|}
\hline $\begin{array}{c}\text { Trial } \\
\text { number }\end{array}$ & $\begin{array}{c}\text { Position 1 } \\
\text { (photons) }\end{array}$ & $\begin{array}{c}\text { Position 2 } \\
\text { (photons) }\end{array}$ & $\begin{array}{c}\text { Position 3 } \\
\text { (photons) }\end{array}$ & $\begin{array}{c}\text { Position 4 } \\
\text { (photons) }\end{array}$ & $\begin{array}{c}\text { Position 5 } \\
\text { (photons) }\end{array}$ \\
\hline 1 & $4.6 \times 10^{5}$ & $5.2 \times 10^{5}$ & $4.7 \times 10^{5}$ & $6.2 \times 10^{5}$ & $4.9 \times 10^{5}$ \\
\hline 2 & $5.8 \times 10^{5}$ & $7.0 \times 10^{5}$ & $5.4 \times 10^{5}$ & $7.1 \times 10^{5}$ & $4.4 \times 10^{5}$ \\
\hline Mean & $\begin{array}{l}(5.2 \\
\pm 0.6) \times 10^{5}\end{array}$ & $\begin{array}{l}(6.1 \\
\pm 0.9) \times 10^{5}\end{array}$ & $\begin{array}{l}(5.0 \\
\pm 0.4) \times 10^{5}\end{array}$ & $\begin{array}{l}(6.6 \\
\pm 0.5) \times 10^{5}\end{array}$ & $\begin{array}{l}(4.6 \\
\pm 0.3) \times 10^{5}\end{array}$ \\
\hline
\end{tabular}

Using the measured values for the pixel area $\left(A_{p}=0.74 \mathrm{~mm}^{2}\right)$ and the OSLD area $\left(A_{D}=7.5 \mathrm{~mm}^{2}\right)$, Equations 3.3 and 5.1 and the data displayed in Table 5.6, we calculated the area-averaged air kerma for the entire image to be $(7.2 \pm 0.4) \mathrm{cGy}$. This value was verified using OSLD calibration data from the National Research Council of Canada. This was done by taking their calibration for Cobalt-60 data and scaling it to $33.17 \mathrm{keV}$ using the mass energy-absorption coefficients for the OSLDs, air and water. It should be noted due to small-beam dosimetry being very difficult that the value listed is likely only good to within a factor of two. This is because typically in dosimetry the beam is of uniform fluence covering the entire area of the detector. In our case the beam 
covered a very small portion of the detector, which results in electrons scattering outside of the target area but none scattering in. Thus an electronic disequilibrium exists and limits the accuracy of our result. 


\section{Chapter 6: Conclusions and}

\section{Future Work}

\subsection{Conclusions}

$\mathrm{X}$-ray projection scatter imaging has several benefits over traditional imaging. The two most important are the potential for increase of contrast between two materials of similar atomic makeup and the ability to generate multiple images from a single data set.

We have developed an automated system which generates coherent scatter projection images by measuring the scatter patterns of a material over the desired area. The primary beam was recorded simultaneously to create transmission images. We have demonstrated that, for projection imaging, the coherent scatter is not only free but is useful information, currently un-utilized in traditional imaging. Multiple pencil beams are required because acquiring a typical image of about 3000 pixels with a single beam in a step-and-shoot setup took over $4 \mathrm{~h}$. To obtain multiple pencil beams a customizable collimator was designed and built to segment a fan beam. To use the information from the detector two disentangling algorithms were developed, with MLEM performing the best for real data. The data displayed in Figure 5.24 took a total of $56 \mathrm{~min}$ to acquire. Including readout and stepping motor translation, the time per shot is $5.8 \mathrm{~s}$.

This 'work only investigated samples up to a few centimetres thick. For thicker phantoms multiple scatter is going to play a larger role, and attenuation (due to the beam paths being different for various locations at the same scattering angle) is going to have a 
greater effect on the MLEM algorithm. For these reasons this work would be most beneficial when applied to imaging which requires both significant soft-tissue contrast and have thin samples. Mammography is the most promising application, since the breast is compressed prior to imaging and fibroglandular tissue and adipose have inherently low contrast in traditional transmission imaging.

We have shown that for these phantoms the soft-tissue contrast is significantly greater in the scatter images than the primary images. The SNR cannot be compared because different detectors were used to measure the primary and scattered photons.

Various porcine and tissue/plastic phantoms were imaged using a variety of beam numbers. Pixels were acquired in a hexagonal lattice to maximize packing efficiency. Significantly greater contrast between pairs of plastics and tissue types was demonstrated with coherent scatter photons over primary photons. This work could ultimately be used to increase soft-tissue contrast, reduce dose or both.

The air kerma for the five-beam setup was measured using OSLDs. This is an important quantity to measure as it will set the guideline for the adjustment of the pixel beam-time.

\subsection{Disentangling algorithm improvements}

To improve the disentangling algorithm a more accurate generate scatter pattern (GSP) routine should be developed. In particular a matrix which accounts for the attenuation from radial profile to scatter pattern could be generated if the attenuation through each path was measured. This attenuation through each path could also be estimated by measuring the attenuation straight through the target over the whole area and estimating 
the actual path as an average of multiple normal-incidence sample paths. This information is available through the primary image. This would not significantly increase computation time and would provide a more accurate disentanglement. Also since the boundaries of the object are known (they can easily be found by looking at the data) the scatter image program should reduce the number of beams when one beam is imaging past the boundary of the sample (and thus its scatter signal is zero). A significant performance increase on the current algorithm could be obtained by compiling the program in C or FORTRAN. Another possibility would be to maximize or minimize some other metrics (besides likelihood-estimation or sum of residuals).

\subsection{Improvements on the current system at the CLS}

\subsubsection{Continuous motion}

Ultimately a system which utilizes continuous motion will be implemented to decrease acquisition time significantly. The five-beam scan of the porcine phantom took 56 min to complete, although x-ray data are only acquired for less than $10 \mathrm{~min}$. Continuous motion, therefore, should reduce the scan time to under $20 \%$ of the current system. To implement this system at the CLS all that must be done is continuous data acquisition while the sample is translated. A rotating shutter should be modified to turn the beam off when translating from one row to the next, to avoid unnecessary dose. A rudimentary implementation was done which continuously acquired data ( 1 exposure per second), while translating the sample at $1 \mathrm{~mm} / \mathrm{s}$. Figure 6.1 is a single beam scatter image (integrating from $2.00^{\circ}$ to $4.00^{\circ}$ ) of a Lucite step phantom of array size $6 \times 100$. The 
bottom row is not quite aligned, likely due to a slight miscalibration in the acquisition LabView program.

\subsubsection{Adjusting beam size}

Currently only portions of the sample are exposed (since the pixel size is larger than the beam size). This results in higher localized dose and zero dose elsewhere. If a living sample was imaged this would increase the probability of harmful effects. Thus the beam size should be reduced to match the pixel size, which can be done by using smaller aluminum spacers. This also has the result of improving spatial resolution. In the vertical direction the beam is focused and the setup should be further off-focus to widen the beam to the pixel size.

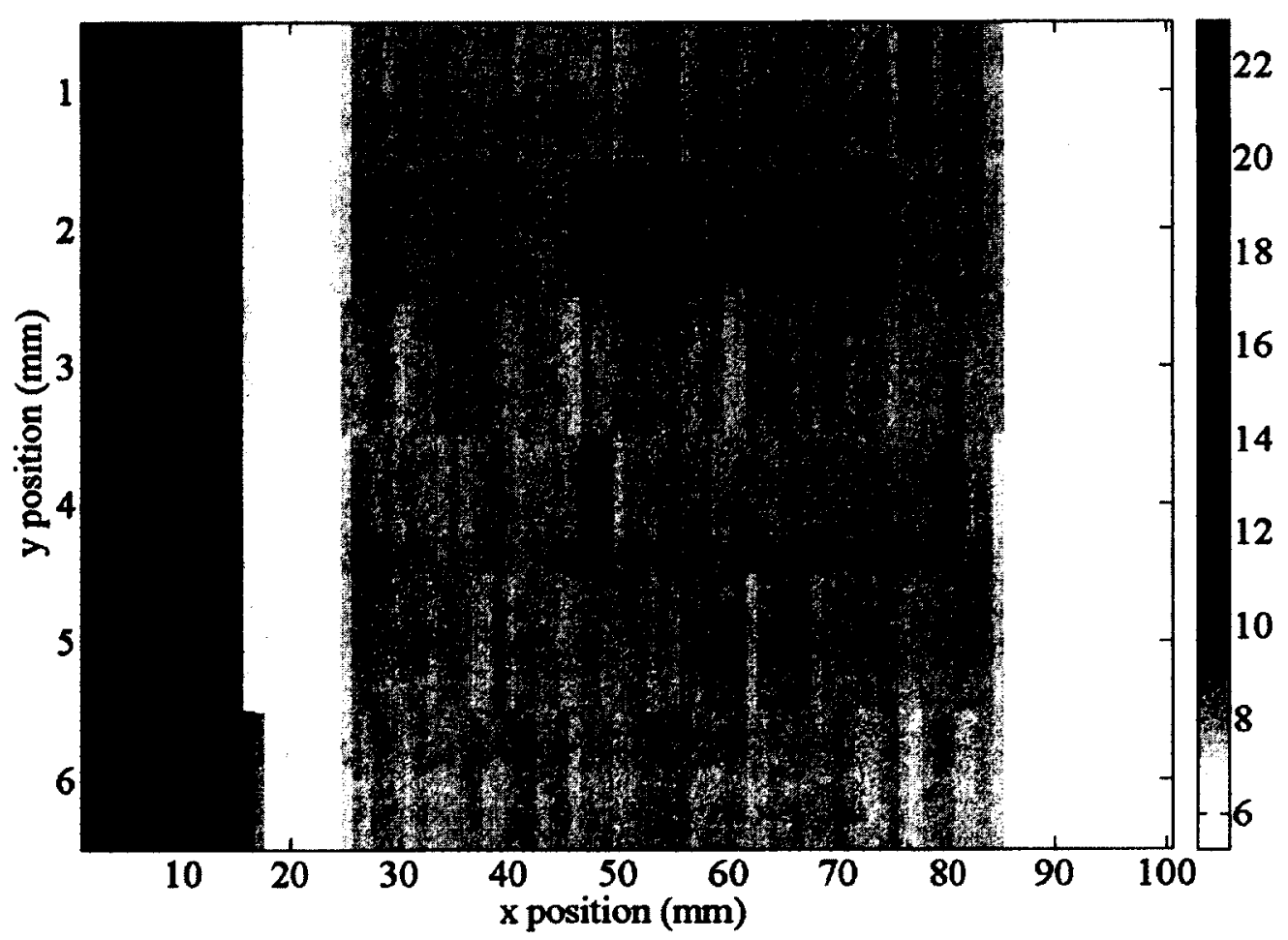

Figure 6.1: Single-beam scatter image of a Lucite step. The data were acquired with continuous motion. The bottom row is slightly misaligned, likely due to a problem in the acquisition software. The pixel array size is $6 \times 100$. 


\subsubsection{Minimizing registration artifacts}

The beam from the monochromator is not perfectly planar (as shown in Figure 3.6) causing registration artifacts to appear in multiple beam images at the boundaries. To account for this the pixels should be assigned to match the actual beam profile. To do this a higher resolution map must be made out of the current image.

\subsubsection{Reducing unwanted scatter}

There was significant unwanted scatter degrading the scatter patterns in the December 2011 experiment which was corrected in the May 2012 experiment by placing sheets of lead above and below the collimator. A sheet of high-attenuating material (such as tungsten-copper) with a window to allow the beams through should be used to eliminate this problem for future experiments.

\subsection{X-ray tube implementation}

The technique of multi-pencil beam projection coherent scatter imaging could be implemented with a standard $x$-ray tube and a flat panel detector. The configuration of pencil beams at the CLS was in a line because the beam there was a fan beam. For a standard $\mathrm{x}$-ray tube the beam will be two dimensional and thus to maximize the used area on the flat panel detector a hexagonal array of beams should be implemented. The collimator designed in this work is specifically for fan beams, so a new collimator to convert the 2D output from the $\mathrm{x}$-ray tube to a series of hexagonally spaced pencil beams will be needed. Conceptually this can be a sheet of tungsten with holes drilled into it, which the beam will travel through. This collimator is, however, not customizable and 
thus a more sophisticated multi-leaf collimator would be needed for clinical applications. The normalization will play a larger role due to the difference in beam intensities caused by $1 / r^{2}$ dependence of photon fluence. All the computer programs in this work do not require the beams to be in a line, only that the centre locations of the beams on the detector are known (which can easily be measured individually). By changing to a polychromatic source there will be a range of $x$, the momentum transfer argument, instead of a single value. This will result in some spectral blurring, degrading the quality of the images. It should be mentioned, however, that since the sample is not infinitesimally thin there is already some blurring due to scatter occurring at different depths. LeClair and Johns ${ }^{42}$ showed that for imaging liver versus fat $10 \mathrm{~mm}$ thick in a $250 \mathrm{~mm}$ spherical water phantom the coherent SNR for a polychromatic $100 \mathrm{kV}$ beam is $87.5 \%$ of the SNR obtained from an optimum monoenergetic beam. This result shows that a monoenergetic beam is not required to obtain high quality scatter images.

\subsection{Kerma}

The air kerma at the phantom entrance was measured under conditions close to free in air using OSLDs and was found to be $(7.2 \pm 0.4) \mathrm{cGy}$. The calibration, however, was done with a $65 \mathrm{kVp}$ unfiltered $\mathrm{x}$-ray beam, not for the actual $33.17 \mathrm{keV}$ monoenergetic beam used for the images. Thus the calibration data (using the calibrated air chamber and the OSLDs) should be acquired at the CLS, to get a more accurate value for the actual air kerma. The CLS calibration should be done in a wide beam (absent from the collimator) to minimize the lack of electronic disequilibrium. This calibration, however, was found to be consistent with the calibration from the National Research Council of Canada although 
the calibration at NRC was done in $50 \mathrm{~mm}$ water, not in air. Other dosimetry options such as TLDs should be investigated.

For comparison the average entrance skin doses for data from the United Kingdom $^{43}$ were $0.15 \mathrm{cGy}$ (skull, lateral), $0.016 \mathrm{cGy}$ (chest, post-anterior), $0.56 \mathrm{cGy}$ (abdomen, abdominal projection) and $0.61 \mathrm{cGy}$ (lumbar spine, abdominal projection). It is clear that our dose is about an order of magnitude larger and thus methods to reduce the dose will be implemented. One possibility is to reduce the exposure time (currently $0.99 \mathrm{~s}$ ) which will also have the benefit of shortening scan duration. This will not be a problem since our images are currently not noise limited. Ultimately in a continuous motion setup we will need to translate the sample sufficiently quickly to make the dose the desired value. 


\section{References}

'H.E. Johns and J.R. Cunningham, The Physics of Radiology (Charles C. Thomas Publisher, Springfield, Illinois, 1983), pp. 144-156.

${ }^{2}$ M.J. Yaffe and P.C. Johns, "Scattered radiation in diagnostic radiology: magnitudes, effects, and methods of reduction," J. Appl. Photogr. Eng. 9, 184-195 (1983).

${ }^{3}$ R.H. Morgan, "An analysis of the physical factors controlling the diagnostic quality of Roentgen images, Part III. Contrast and the intensity distribution function of a Roentgen image," Am. J. Roentgenol. 55, 67-89 (1946).

${ }^{4}$ J.W. Motz and M. Danos, "Image information content and patient exposure," Med. Phys. 5, 8-22 (1978).

${ }^{5}$ L.R.M. Morin and A. Berroir, "Calculation of $x$-ray single scattering in diagnostic radiology," Phys. Med. Biol. 28, 789-797 (1983).

${ }^{6}$ P.C. Johns and M.J. Yaffe, "Coherent scatter in diagnostic radiology," Med. Phys. 10, 40-50 (1983).

${ }^{7}$ E.P. Muntz, T. Fewell, R. Jennings and H. Bernstein, "On the significance of very small angle scattered radiation to radiographic imaging at low energies," Med. Phys. 10, 819823 (1983). 
${ }^{8}$ G. Harding, J. Kosanetzky and U. Neitzel, "Elastic scatter computed tomography," Phys. Med. Biol. 30, 183-186(1985).

${ }^{9}$ G. Harding and J. Kosanetzky, "Status and outlook of coherent x-ray scatter imaging," $J$. Opt. Soc. Am. A 4, 933-944 (1987).

${ }^{10}$ R.D. Speller and J.A. Horrocks, "Photon scattering-a 'new' source of information in medicine and biology?" Phys. Med. Biol. 36, 1-6 (1991).

${ }^{11}$ R.D. Luggar, W.B. Gilboy and N. MacCuaig, "Industrial potential of Rayleigh scattered x-rays for identification of low-Z materials," Proc. SPIE 2092, 378-386 (1993).

${ }^{12}$ R.D. Speller, J.A. Horrocks and R. Lacey, "X-ray scattering signatures for material identification," Proc. SPIE 2092, 366-377 (1993).

${ }^{13} \mathrm{G}$. Harding, "Optimization criteria for CXRS baggage inspection," Proc. SPIE 2511, 64-70 (1995).

${ }^{14} \mathrm{H}$. Strecker, "Automatic detection of explosives in airline baggage using elastic x-ray scatter," Medica Mundi 42, 30-33 (1998).

${ }^{15} \mathrm{G}$. Harding, "Effective density and atomic number determined from diffraction profiles," Proc. SPIE 6319, 631910-1 to 631910-9 (2006). 
${ }^{16} \mathrm{G}$. Harding, H. Fleckenstein, S. Olesinski and G. Zienert, "Liquid detection trial with $\mathrm{x}-$ ray diffraction," Proc. SPIE 7806, 78060G-1 to 78060G-8 (2010).

${ }^{17}$ R.W. Madden, J. Mahdavieh, R.C. Smith and R. Subramanian, “An explosive detection system for airline security using coherent x-ray scattering technology," Proc. SPIE 7079, 707915-1 to 707915-11 (2008).

${ }^{18}$ J.A. Grant, M.J. Morgan, J.R. Davis, D.R. Davies and P. Wells, "X-ray diffraction microtomography," Meas. Sci. Technol. 4, 83-87 (1987).

${ }^{19}$ M.S. Westmore, A. Fenster and I.A. Cunningham, "Angular-dependent coherent scatter measured with diagnostic x-ray image intensifier-based imaging system," Med. Phys. 23, 723-733 (1996).

${ }^{20}$ M.S. Westmore, A. Fenster and I.A. Cunningham, "Tomographic imaging of the angular-dependent coherent-scatter cross section," Med. Phys. 24, 3-10 (1997).

${ }^{21}$ D.L. Batchelar and I.A. Cunningham, "Material-specific analysis using coherent-scatter imaging," Med. Phys. 29, 1651-1660 (2002).

${ }^{22}$ D.L. Batchelar, M.T.M. Davidson, W. Dabrowski and I.A. Cunningham, "Bonecomposition imaging using coherent-scatter computed tomography: Assessing bone health beyond bone mineral density," Med. Phys. 33, 904-915 (2006). 
${ }^{23}$ S.R. Beath and I.A. Cunningham, "Pseudomonoenergetic x-ray diffraction measurements using balanced filters for coherent-scatter computed tomography," Med. Phys. 36, 1839-1847 (2009).

${ }^{24}$ R.J. LeClair, M.M. Boileau and Y. Wang, "A semianalytic model to extract differential linear scattering coefficients of breast tissue from energy dispersive $\mathrm{x}$-ray diffraction measurements," Med. Phys. 33, 959-967 (2006).

${ }^{25}$ C. Cui, S.M. Jorgensen, D.R. Eaker and E.L. Ritman, "Coherent $\mathrm{x}$-ray scattering for discriminating bio-compatible materials in tissue scaffolds," Proc. SPIE 7078, 70781S-1 to $70781 \mathrm{~S}-10$ (2008).

${ }^{26}$ T.W. Wysokinski, D. Chapman, G. Adams, M. Renier, P. Suortti and W. Thomlinson, "Beamlines of the biomedical imaging and therapy facility at the Canadian light sourcePart 1," Nucl. Instr. and Meth. Phys. Res. A 582, $73-76$ (2007).

${ }^{27}$ J. Cutler, E. Hallin, M. de Jong, W. Thomlinson and T. Ellis, "The Canadian Light Source: The newest synchrotron in the Americas," Nucl. Instr. and Meth. Phys. Res. A 582, 11-13 (2007).

${ }^{28}$ R.J. LeClair and P.C. Johns, "A semianalytic model to investigate the potential applications of x-ray scatter imaging," Med. Phys. 25, 1008-1020 (1998). 
${ }^{29}$ M.Z. Hasan and P.C. Johns, "Energy-dispersive technique to measure $\mathrm{x}$-ray scattering form factors over a wide momentum transfer range," Physics in Canada 60, 145 (2004) (abstract).

${ }^{30} \mathrm{~B}$. W. King and P.C. Johns, "An energy-dispersive technique to measure $\mathrm{x}$-ray coherent scattering form factors of tissues," Phys. Med. Biol. 55, 855-871 (2010).

${ }^{31}$ B.W. King, K.A. Landheer and P.C. Johns, "X-ray coherent scattering form factors of tissues, water and plastics using energy dispersion," Phys. Med. Biol. 56, 4377-4397 (2011).

${ }^{32}$ M. Nisar and P.C. Johns, "Coherent scatter x-ray imaging of plastic/water phantoms," Proc. SPIE 5578, 445-453 (2004).

${ }^{33}$ P.C. Johns, B.E. Bewer and L.D. Chapman, "Initial experiments in monoenergetic x-ray scatter imaging at the BioMedical Imaging and Therapy facility of the Canadian Light Source," Med. Phys. 36, 4306 (2009) (abstract).

${ }^{34} \mathrm{~K}$. Landheer and P.C. Johns, "Coherent scatter ring integration imaging," Med. Phys. 38, 3439 (2011) (abstract).

${ }^{35} \mathrm{~K}$. Landheer and P.C. Johns, "Coherent x-ray scatter projection imaging using an array of monoenergetic pencil beams," Proc. SPIE 8358, 835810-1 to $835810-10$ (2012). 
${ }^{36}$ M.J. Berger, J.H. Hubbell, S.M. Seltzer, J. Chang, J.S. Coursey, R. Sukumar, D.S. Zucker and K. Olsen. XCOM: Photon Cross Section Database (version 1.5) (2010). [Online] Available: http://physics.nist.gov/xcom [Tuesday, 17-Jul-2012 16:56:13 EDT]. National Institute of Standards and Technology, Gaithersburg, MD.

${ }^{37}$ P.C. Johns, R.J. LeClair and M.P. Wismayer, "Medical x-ray imaging with scattered photons," SPIE Technical Digest (Opto-Canada) TD01, 355-357 (2002).

${ }^{38}$ P.C. Johns and M.P. Wismayer, "Measurement of coherent x-ray scatter form factors for amorphous materials using diffractometers," Phys. Med. Biol. 49, 5233-5250 (2004).

${ }^{39}$ A. Scullion, E. Yukihara and G. Sawakuchi, "Construction of a cost-effective and versatile Optically Stimulated Luminescence Detector (OSLD) reader for research," Med. Phys. 38, 3503 (2011) (abstract).

${ }^{40}$ L. Botter-Jensen, S.W.S. McKeever and A.G. Wintle, Optically Stimulated Luminescence Dosimetry (Elsevier Science B.V., Amsterdam, The Netherlands, 2003), p. 75.

${ }^{41} \mathrm{~K}$. Lange and R. Carson, "EM reconstruction algorithms for emission and transmission tomography," J. Comp. Assist. Tomo. 8, 306-316 (1984). 
${ }^{42}$ R.J. LeClair and P.C. Johns, "Analysis of spectral blur effects in x-ray scatter imaging," Med. Phys. 26, 1811-1816 (1999).

${ }^{43}$ W. Huda, "Medical Radiation Dosimetry," pp. 29-40 in From Invisible to Visible - The Science and Practice of X-ray Imaging and Radiation Dose Optimization, edited by D.P. Frush and W. Huda (Radiological Society of North America, Inc, Oak Brook, Illinois, 2006). Refer to Table 5. 


\section{Appendix}

The codes in this Appendix are the important MATLAB programs to create a coherent scatter image from a series of scatter patterns. The code is run by calling klscatterImageMultiBeam, which calls klscatterPixelMultiBeam to calculate the pixel value from the scatter pattern. Within klScatterPixelMultiBeam a function klImageFilter is called which filters the scatter patterns to remove detector artifacts. Finally, klhexagonalDisplay is called from within klscatterImageMultiBeam to display the actual image in a hexagonal lattice. The files from the flat panel detector should be input as tab delimited text files (.txt).

\section{A.1 klscatterImageMultiBeam}

This program takes in the base filename (truncates the exact number of the text file, i.e., if an image from the data contained in

"118_H9252_apple_twobeam_threespacing_3300ms_70x64_2011Dec5_2173.txt" is desired then the filename should be input as

"118_H9252_apple_twobeam_threespacing_3300ms_70x64_2011Dec5") and returns the coherent scatter image using the input angular range.

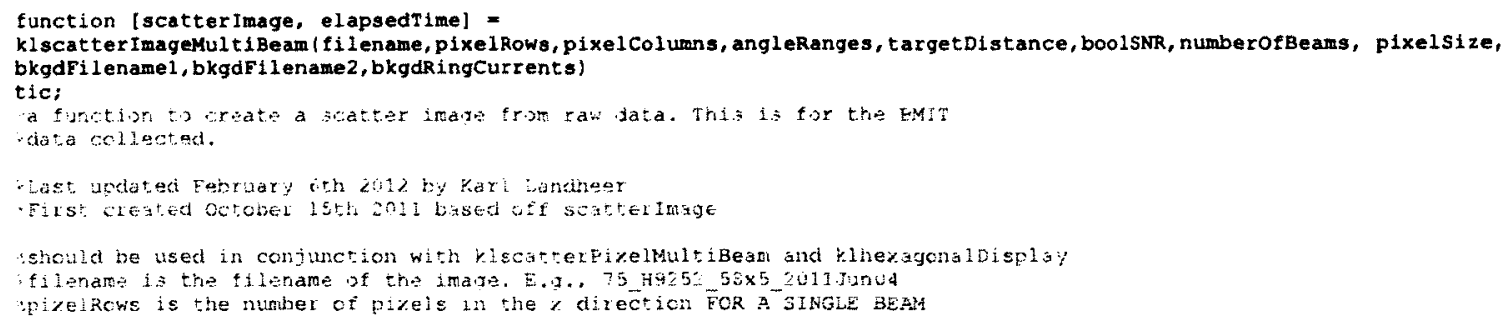




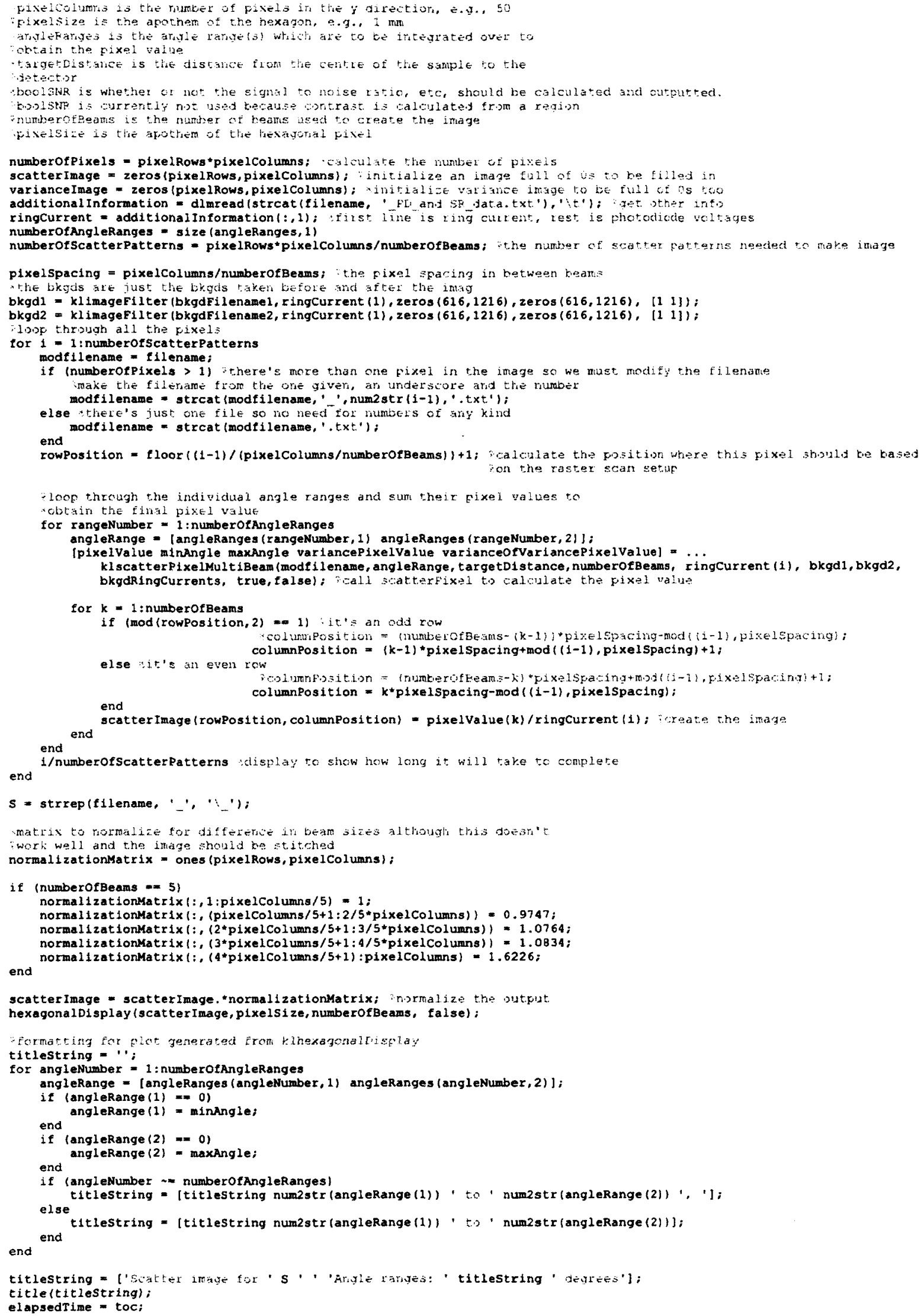




\section{A.2 klscatterPixelMultiBeam}

This is the program which contains the MLEM algorithm. This function essentially takes the filename where the scatter image is contained and returns an array of pixel values. It makes use of the subroutine klimageFilter which is shown in Appendix A.3.

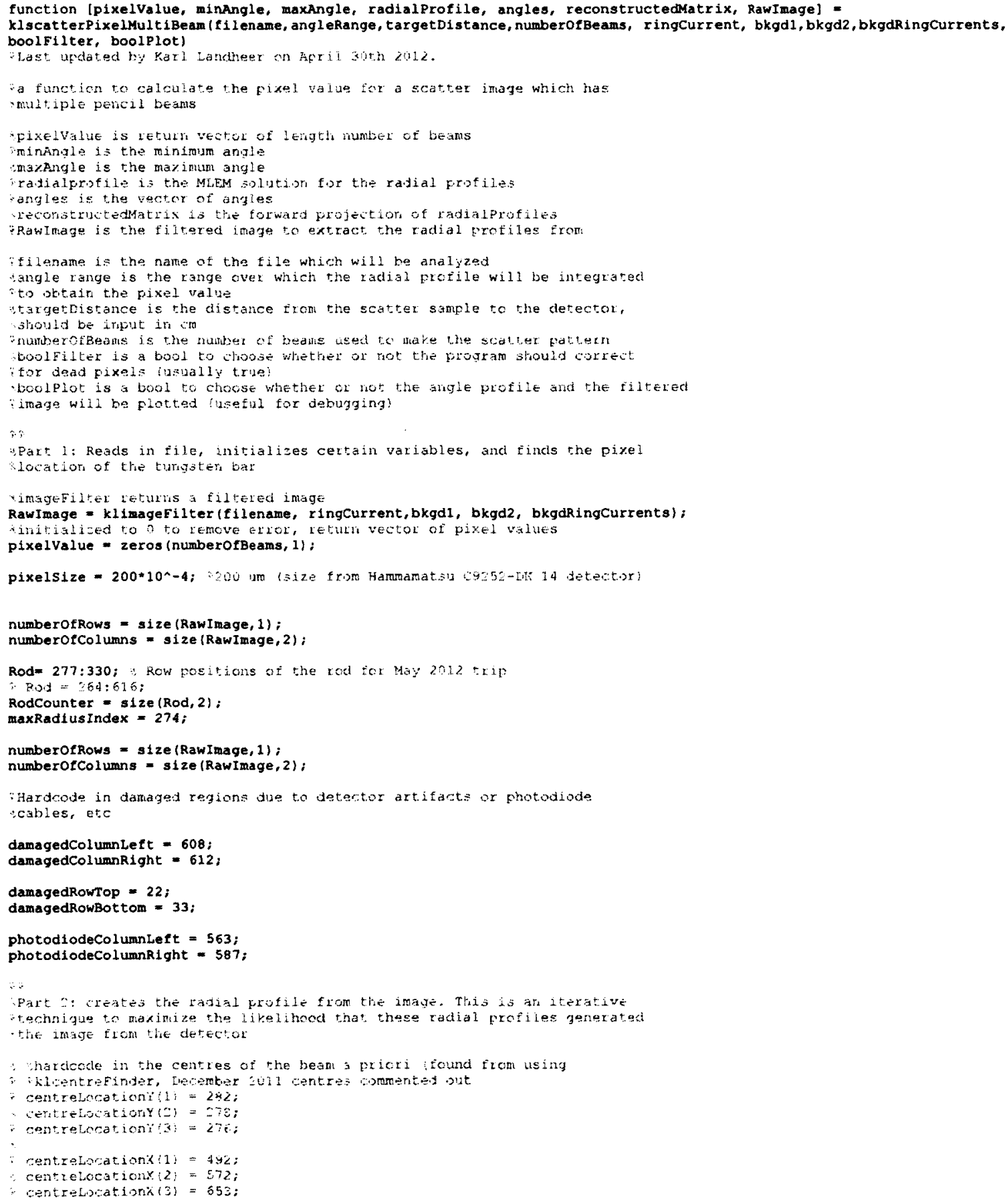




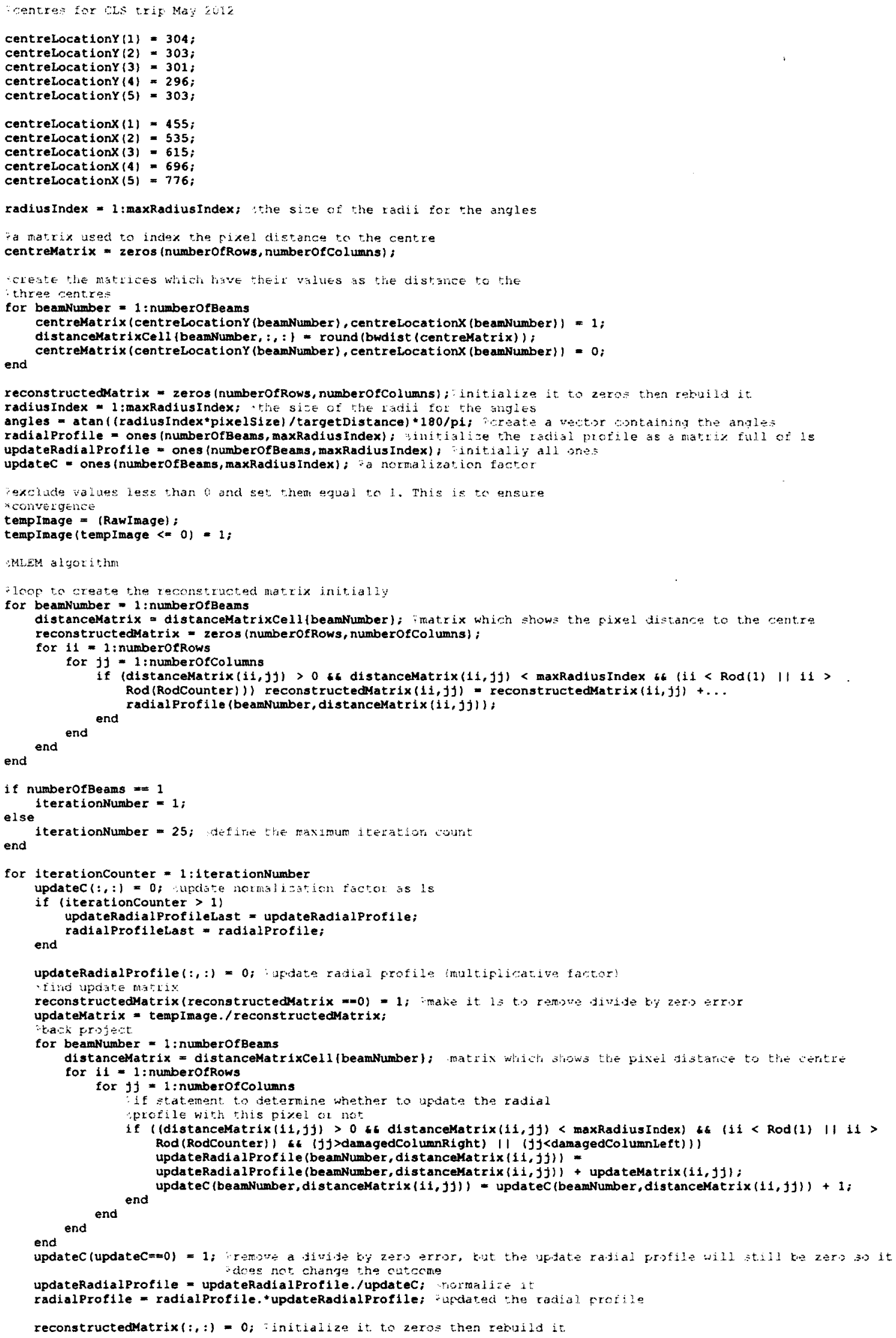

reconstructedMatrix = zeros (numberofRows, numberotcolumns); initialize it to zeros then rebuild it 


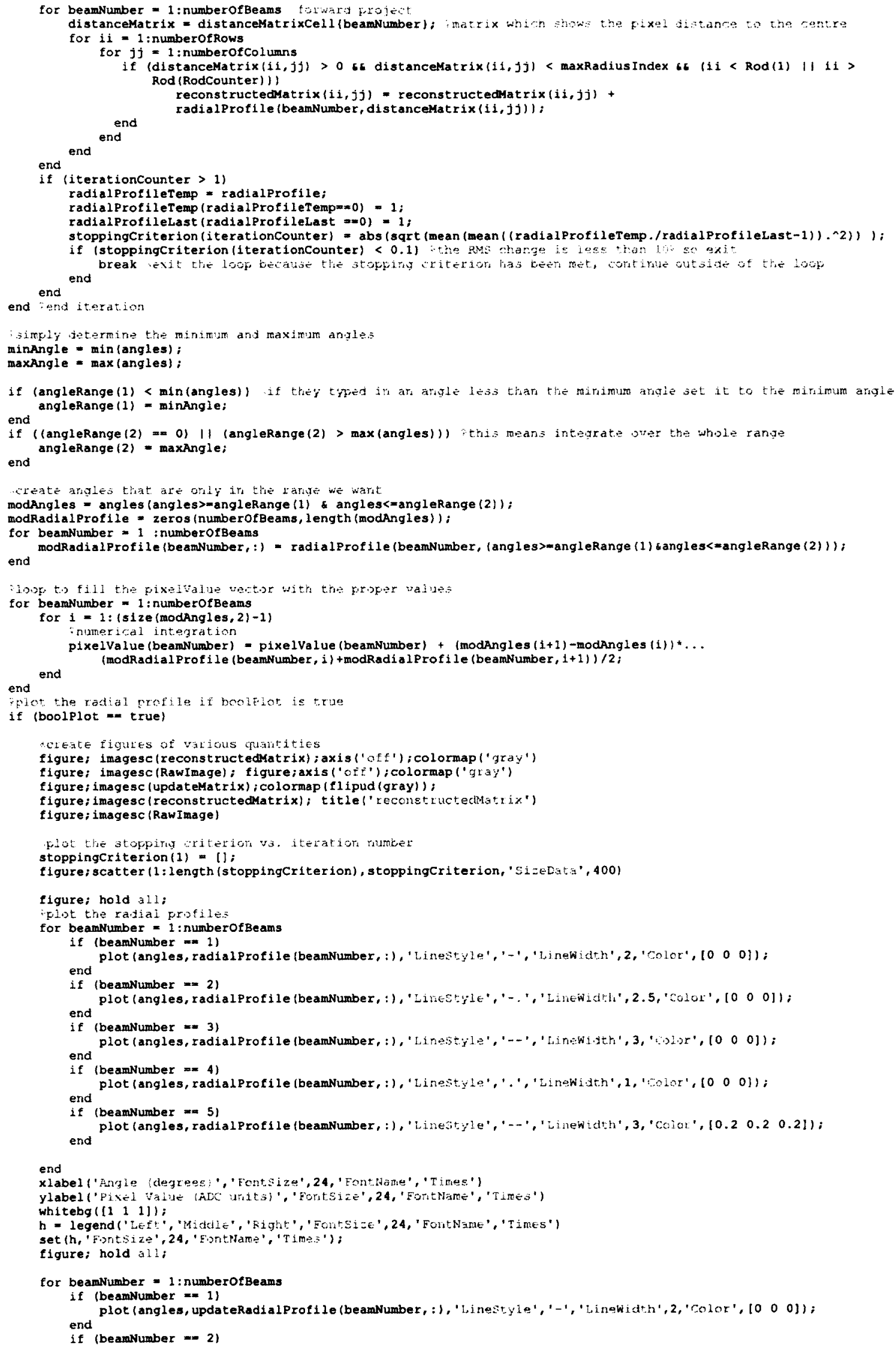




\section{A.3 klimagefilter}

This is an image filtering program which takes the filename of the scatter pattern and returns a filtered scatter pattern. It currently applies only a median filter, the code containing the high-pass filter is commented out because it was not necessary for the later

\section{clean images.}

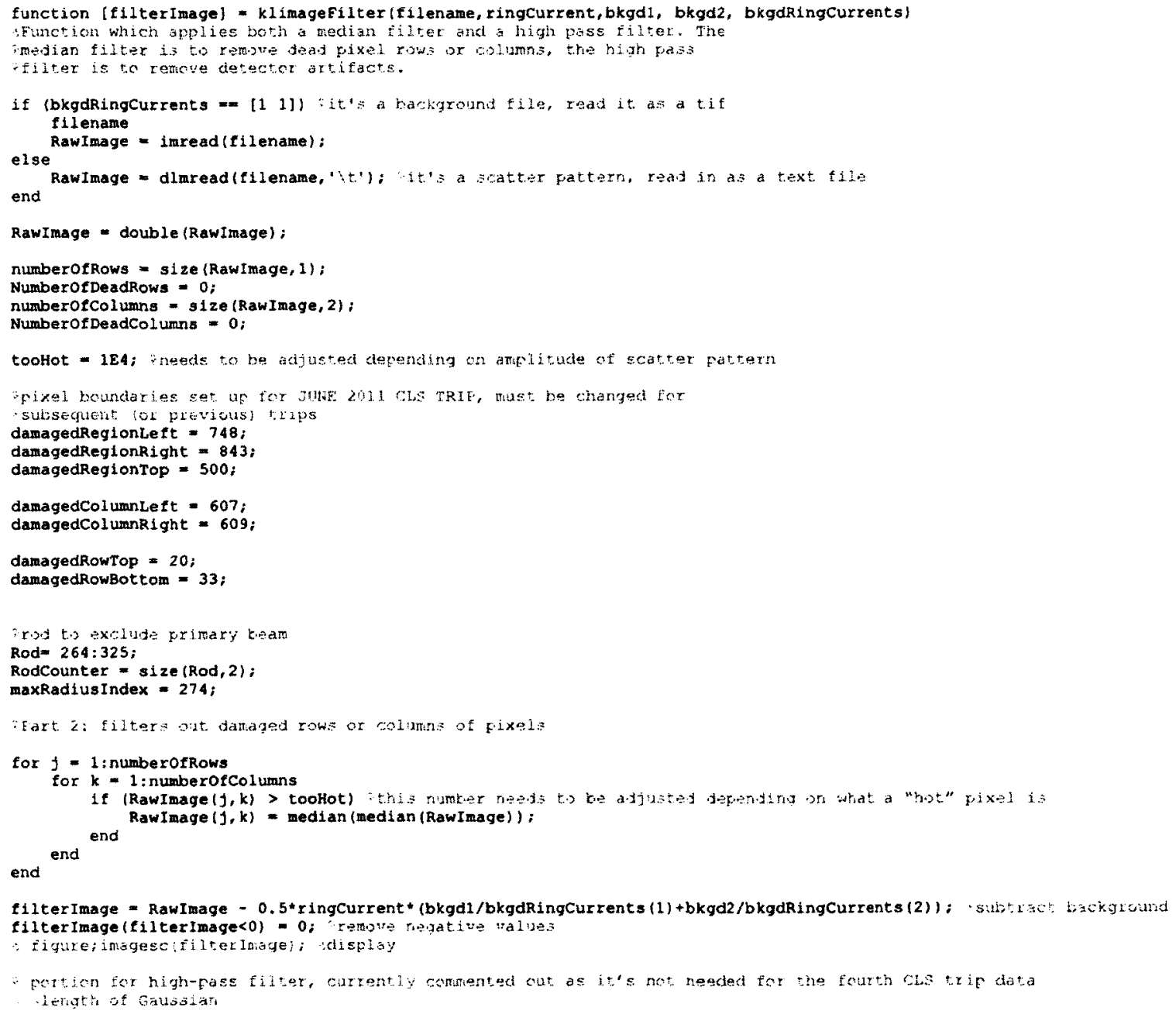




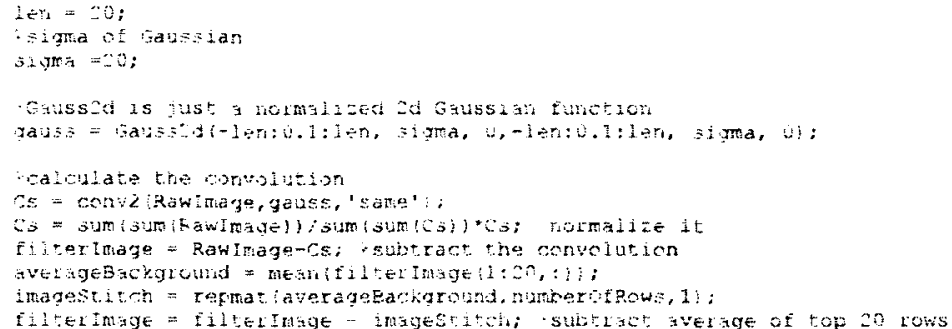

\section{A.4 klhexagonalDisplay}

This program simply takes a matrix and creates an image by placing the pixels in a hexagonal lattice. It also has the capability of leveling and windowing the data by truncating the data that falls above or below a certain limit, and automatically spanning the full gray space of the image. This is to be used if the contrast between two regions in two different images should be held constant (for display purposes).

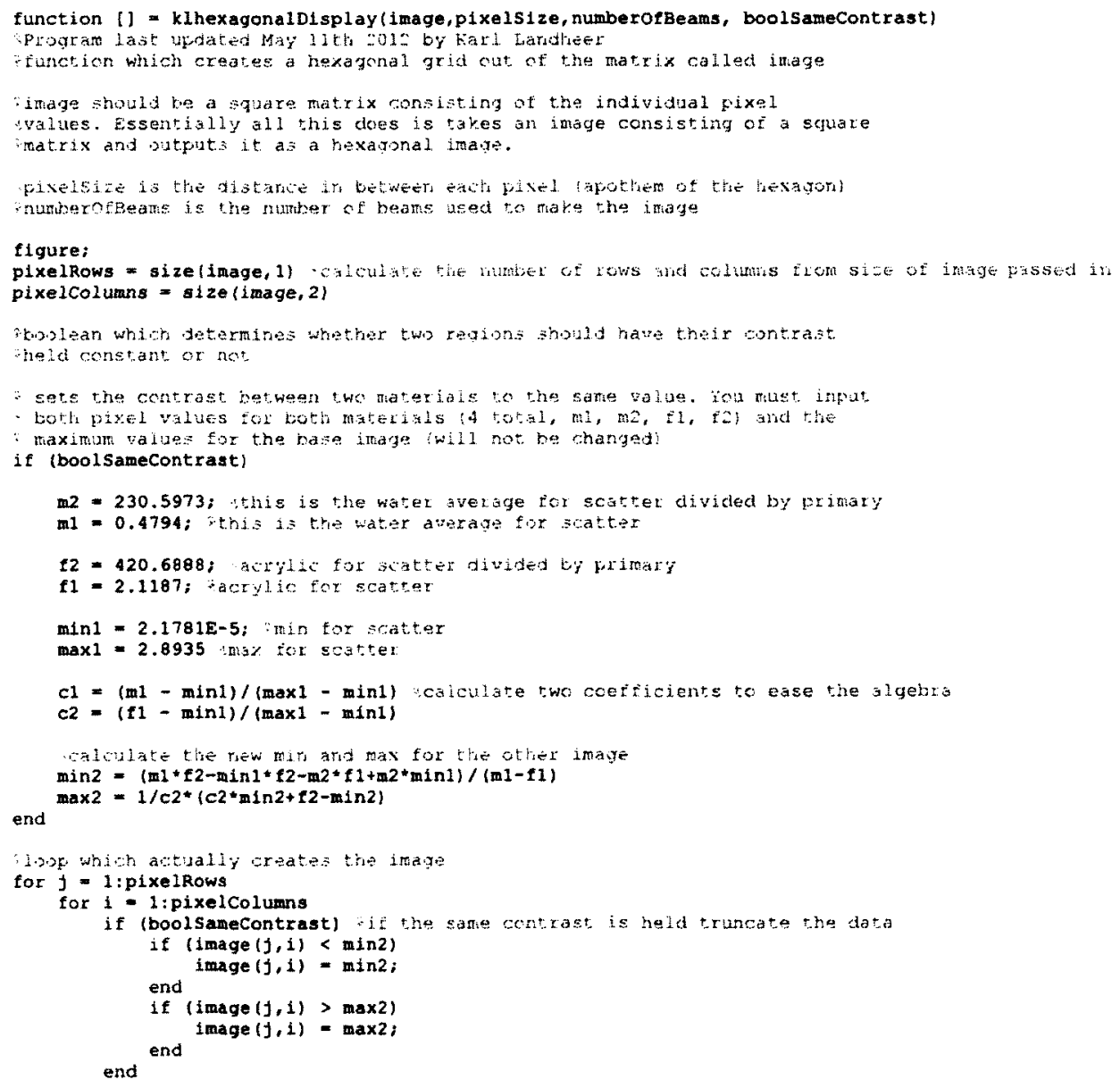




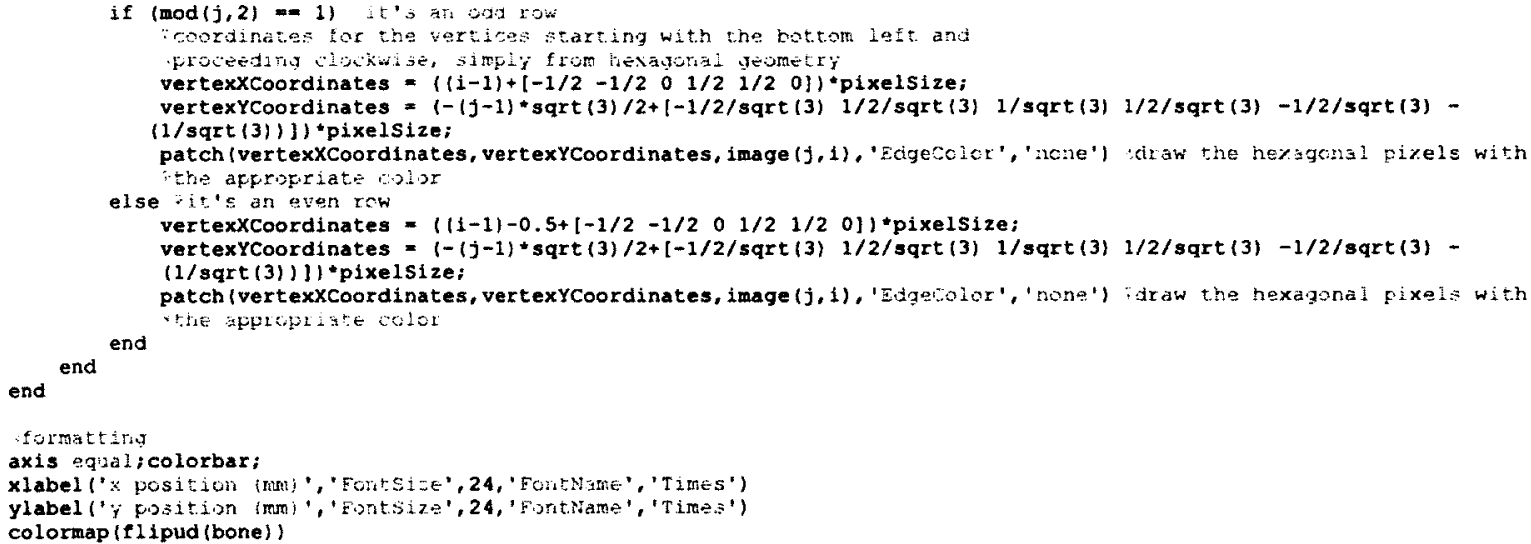

